\title{
WestVirginiaUniversity
}

THE RESEARCH REPOSITORY @ WVU

Graduate Theses, Dissertations, and Problem Reports

2010

\section{Sleep and sleepiness among first-time postpartum parents}

\author{
Salvatore Insana \\ West Virginia University
}

Follow this and additional works at: https://researchrepository.wvu.edu/etd

\section{Recommended Citation}

Insana, Salvatore, "Sleep and sleepiness among first-time postpartum parents" (2010). Graduate Theses, Dissertations, and Problem Reports. 4608.

https://researchrepository.wvu.edu/etd/4608

This Dissertation is protected by copyright and/or related rights. It has been brought to you by the The Research Repository @ WVU with permission from the rights-holder(s). You are free to use this Dissertation in any way that is permitted by the copyright and related rights legislation that applies to your use. For other uses you must obtain permission from the rights-holder(s) directly, unless additional rights are indicated by a Creative Commons license in the record and/ or on the work itself. This Dissertation has been accepted for inclusion in WVU Graduate Theses, Dissertations, and Problem Reports collection by an authorized administrator of The Research Repository @ WVU.

For more information, please contact researchrepository@mail.wvu.edu. 


\title{
Sleep and Sleepiness Among First-Time Postpartum Parents
}

Salvatore Insana, M.S.

\author{
Dissertation submitted to the \\ Eberly College of Arts and Sciences at \\ West Virginia University \\ in partial fulfillment of the requirements \\ for the degree of
}

\author{
Doctor of Philosophy \\ in \\ Psychology
}

\author{
Hawley Montgomery-Downs, Ph.D., Chair \\ Heather Clawges, M.D. \\ Amy Fiske, Ph.D. \\ Katherine Karraker, Ph.D. \\ JoNell Strough, Ph.D.
}

\author{
Department of Psychology \\ Morgantown, West Virginia \\ 2010
}

Keywords: sleep, sleepiness, postpartum, mother, father, Multiple Sleep Latency Test, actigraphy, hand-held computers 


\title{
ABSTRACT \\ Sleep and Sleepiness Among First-Time Postpartum Parents
}

\author{
Salvatore P. Insana, M.S.
}

OBJECTIVES: Postpartum maternal sleep disturbance is common. However, little research has examined postpartum fathers' sleep. Additionally, the magnitude of postpartum mothers' and fathers' daytime sleepiness has not been objectively indexed. The aims of this dissertation study were to: 1) objectively examine the difference in sleep and sleepiness levels among first-time mothers and fathers during their early postpartum period; 2) examine parents' perceptions of their own and their partner's mood, sleep, and sleepiness.

METHOD: Twenty one first-time postpartum mother-father dyads ( $N=42 ; 27.38 \pm 4.92$ years; $91.67 \%$ white; $15.40 \pm 3.53$ years of education; annual household income $\$ 56,091 \pm \$ 34,320$ ), completed one week of continuous wrist actigraphy and electronic sleep diary monitoring followed by standard four-nap Multiple Sleep Latency Test (MSLT) when their infants were 6.93 \pm 1.26 weeks old.

RESULTS: Mothers $(M=424.83, S D=42$ minutes $)$ had significantly $(F[1,18]=17.31, p<.01$, $d=1.30)$ more 24-hour sleep time than fathers $(M=375.14, S D=34.06$ minutes $)$; however, mothers $(M=18.86, S D=3.34)$ also had significantly $(F[1,18]=13.17, p<.01, d=1.12)$ more sleep fragmentation than fathers $(M=14.34$, $S D=4.62)$. Fathers $(M=11.80, S D=4.60)$ had significantly $(F[1,19]=11.85, p<.005, d=0.88)$ higher levels of sleepiness than mothers $(M=$ 8.03, $S D$ = 3.92). Overall, fathers were better at reporting their partner's objective sleepiness than mothers were their partner's; furthermore, fathers were better judges of their own objective nocturnal wake time than mothers were their own.

CONCLUSIONS: The current study is the first to objectively report sleepiness values among postpartum mothers and fathers during their early postpartum period. Furthermore, the current study expands the knowledge of the relatively unknown sleep experience of postpartum fathers. Additionally, the identification of parental perceptions of sleep and sleepiness may lay the framework to identify ways to maximize productivity and safety within a postpartum household. The early postpartum period is important in the context of these findings because it is a time when one, or both parents go back to work to function as productive members of society - and they have a new infant to care for-yet, new parents may experience various sleep and sleepiness-associated impairments.

SUPPORT: NIH grant R21HD053836 (HMD); APA Basic Psychological Science Research Grant (SI); WVU Doctoral Student Research Support (SI); WVU Alumni Fund (SI); WVU Behavioral and Biomedical Sciences Training Scholarship Research Award (SI). 


\section{ACKNOWLEDGEMENTS}

I sincerely thank the members of my dissertation committee: Heather Clawges, M.D.; Amy Fiske, Ph.D.; Katherine Karraker, Ph.D.; Hawley Montgomery-Downs, Ph.D.; and JoNell Strough, Ph.D. Their conceptual and editorial feedback was an essential component to my successful completion of this great milestone and the development of this scientific work.

I especially thank my dissertation chair, advisor, and mentor, Dr. Hawley MontgomeryDowns for her multitude of contributions to this project. Notably, Dr. Montgomery-Downs is responsible for my development as a scientist and a critical thinker. I give her my deepest gratitude for guiding me through the process of successfully obtaining a Doctor of Philosophy; the course was a life-changing experience. The last page of this document is dedicated to my academic lineage's tradition of adding a family recipe to one's final dissertation document.

My gratitude and respect extends to the mothers, fathers, and their infants who participated in this dissertation study. The time and effort that they donated from their busy and tireless lives to propagate science was invaluable. Their participation in this research was over and beyond a thankless contribution to society; it was also, a central component to extend current scientific knowledge to a new level that will ultimately improve the family functioning of future generations.

The current research was conducted with the assistance from Megan Clegg-Kraynok, M.S.; Chelsea Costello; Laura Mancini, B.S.; and Eleanor Santy, B.A. Their unselfish contributions of time and effort to assist me with data collection were of significant importance to my completion of this work. Katherine Karraker, Ph.D. and Ryan Anderson, M.S. generously provided accommodations for the participants who visited the Sleep Research Laboratory.

A personal note of gratitude goes out to Drs.: Brian Ayotte, Eric Goedereis, Matthew Goldfine, and Clare Mehta; as well as, Megan Clegg-Kraynok, Joey Kraynok, Alisa O’Riley, Sarah Stahl, Amanda Wheat, and Marion Young for their friendship and support throughout graduate school. Dr. Eric Goedereis deserves a special note of appreciation for his feedback on various stages of this work, as well as a wide range of supportive contributions.

Words cannot describe the gratitude that my family deserves. My mother, father, sisters, and brother have been a solid support network. My academic accomplishments, as represented by this document, would have been unobtainable without their consistent support, advice, and inspiration. A special acknowledgement goes out to my parents for their resilience through the sleepless nights that my siblings and I caused throughout our development.

The current work was funded by: NIH grant R21HD053836 (HMD); T32GM081741 Behavioral and Biomedical Sciences Training Scholarship (SI); APAGS Basic Psychological Science Research Grant (SI); WVU Doctoral Student Research Support (SI); and the Department of Psychology Alumni Fund (SI). I was able to disseminate multiple layers of this project to various regional, national, and international audiences with the travel support provided by the: NIH, NICHD, and Sackler Institute; WVU Psychology Department; and Eberly College of Arts and Sciences.

Thank you all! 


\section{TABLE OF CONTENTS}

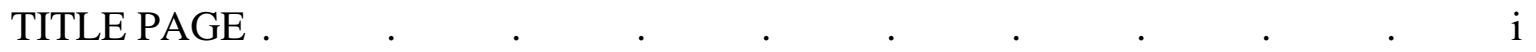

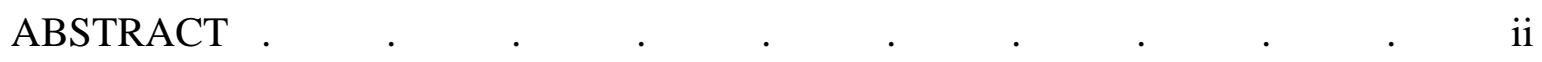

ACKNOWLEDGEMENTS ..$\quad$. $\quad . \quad$. . . . . . .

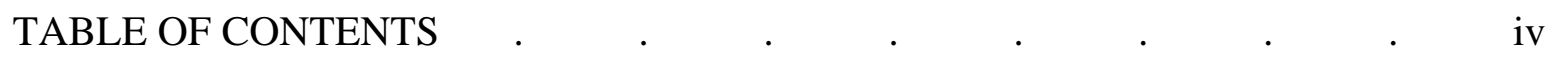

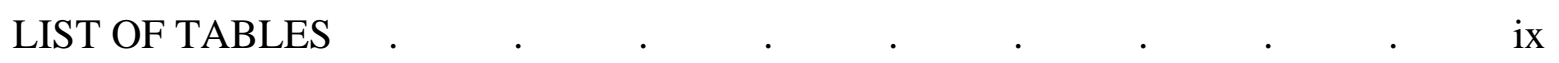

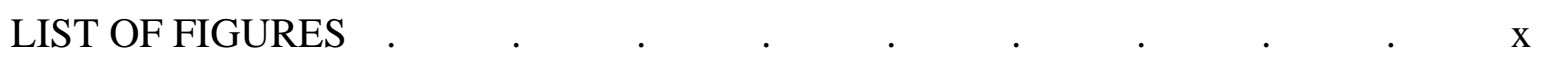

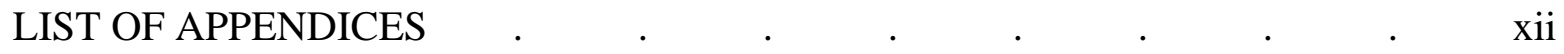

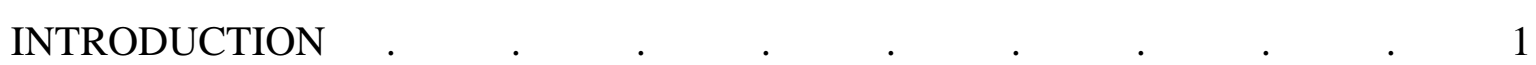

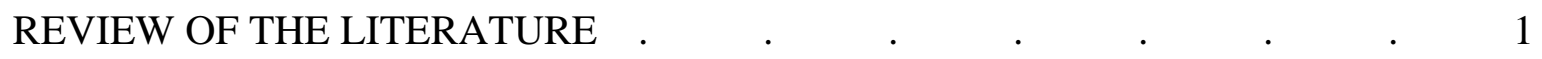

Sleep Throughout the Postpartum Period $\quad$. $\quad$. $\quad$. $\quad$. $\quad$. 1

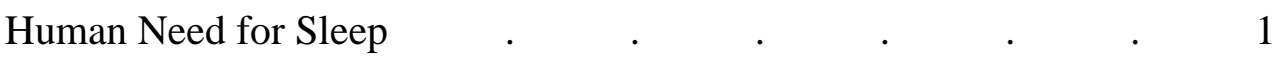

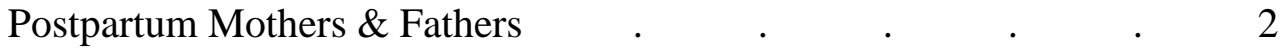

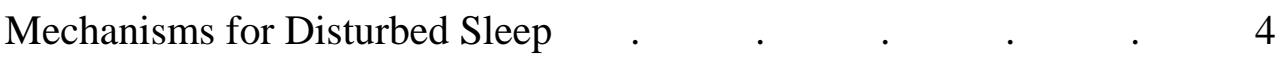

Postpartum Physiological Alterations. $\quad$. $\quad$. $\quad$. 4

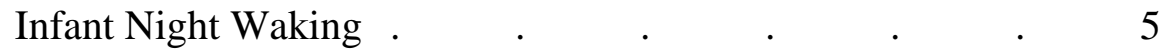

Caregiving and Mother / Father Relationships . . 5

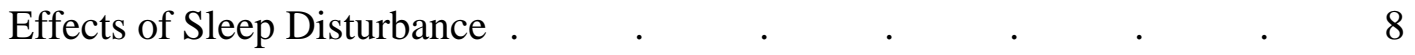

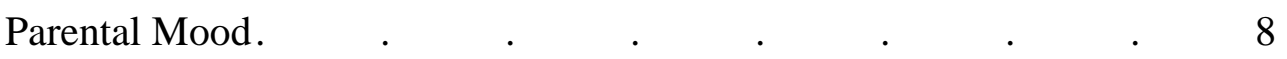

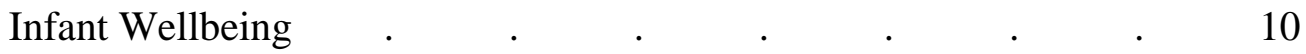

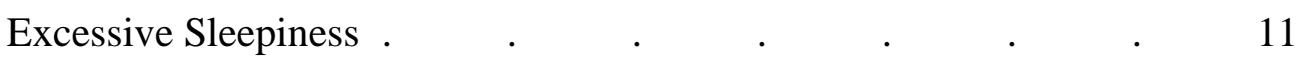

Overview of Objective Sleep Measurement Methods $\quad$ • $\quad$ • $\quad$. $\quad 12$

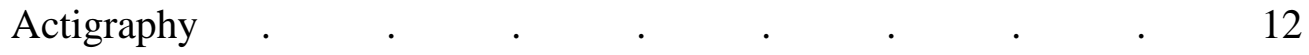




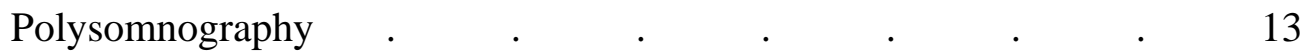

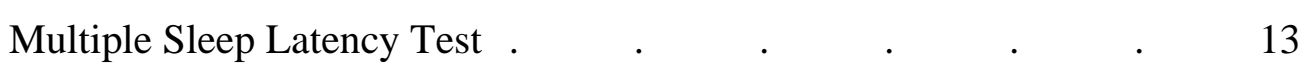

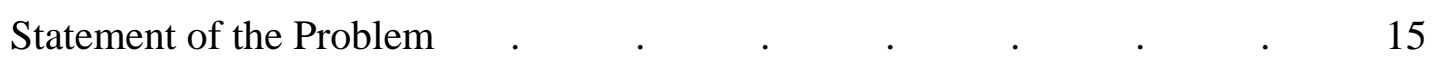

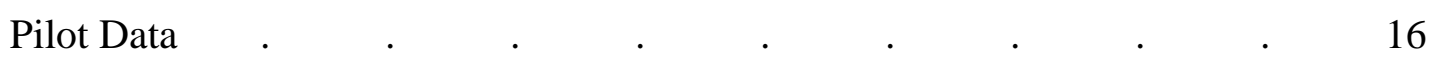

Research Questions (RQ) and Hypotheses (H) $\quad$. $\quad \quad \quad \quad \quad$. $\quad 16$

Aim1: Research Questions for Objective Sleep and Sleepiness Data 17

Aim 2: Research Questions for Subjective Sleep Data $\quad$ 19

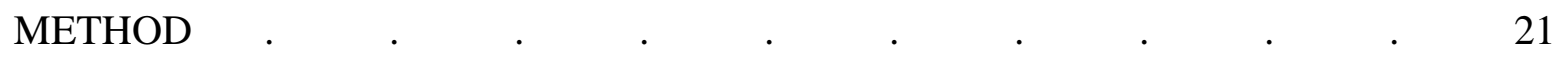

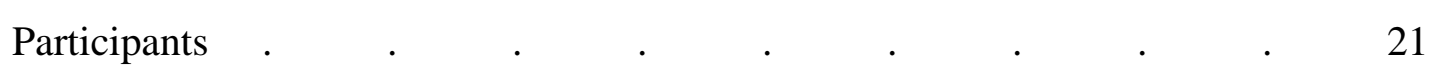

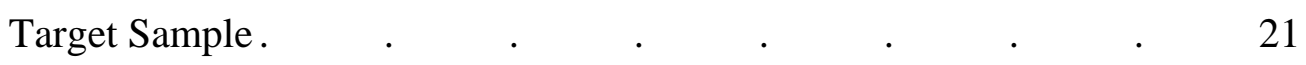

Target power for actigraphy analyses. $\quad$. $\quad$. $\quad$. 22

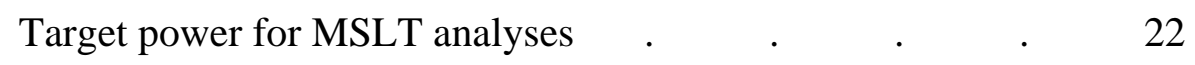

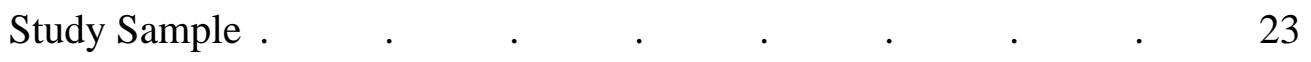

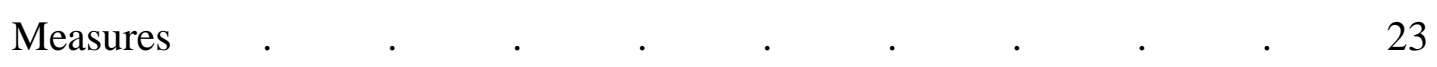

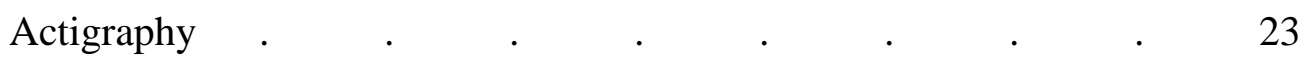

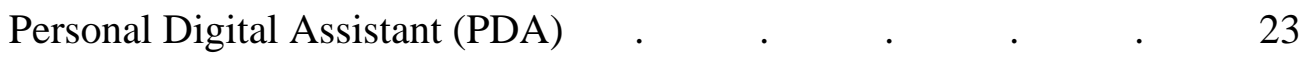

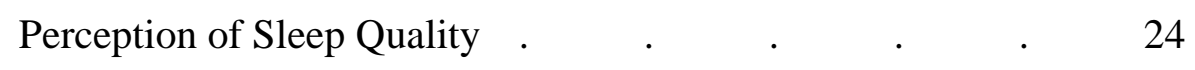

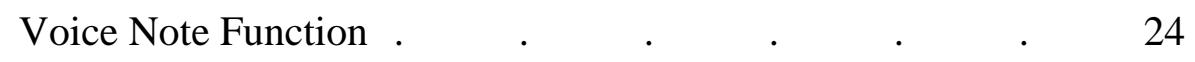

Sleep and Watch Diaries $\quad . \quad$. $\quad . \quad$. $\quad$. $\quad 24$

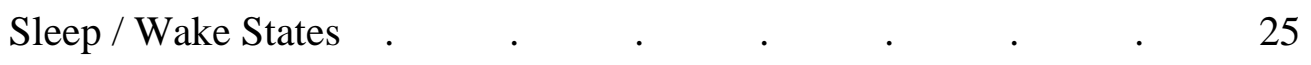

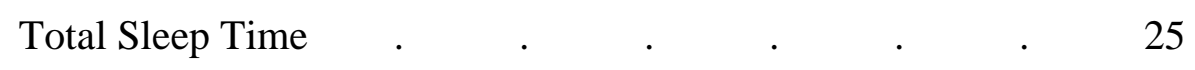

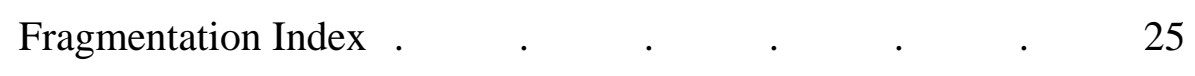

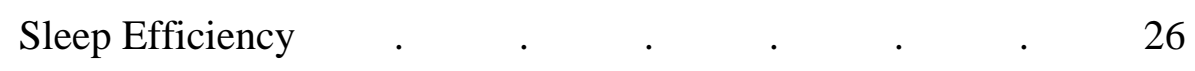




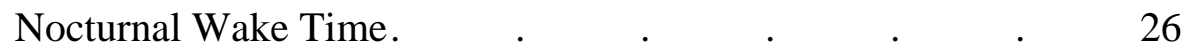

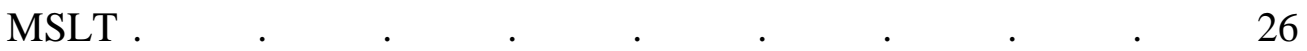

Subjective Rating of Sleep Onset Latency $\quad$ • $\quad$. $\quad$. $\quad$. $\quad 29$

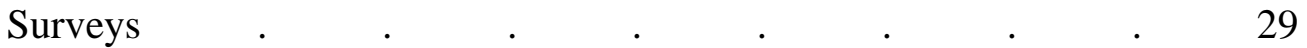

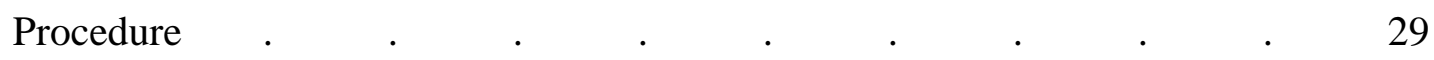

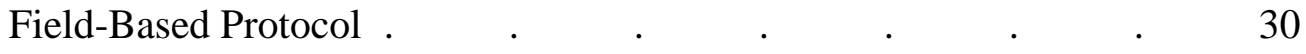

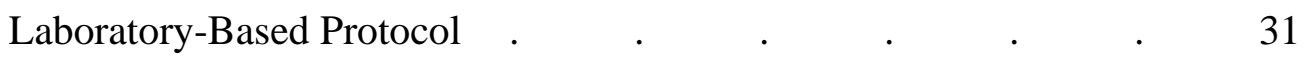

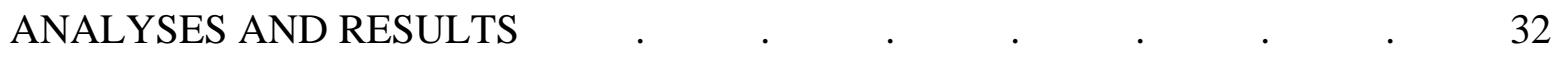

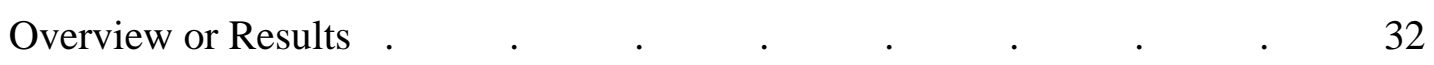

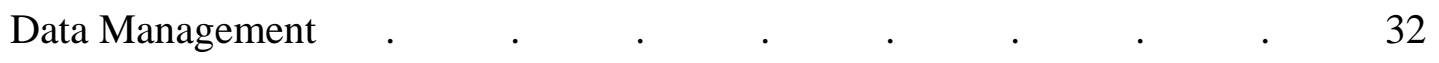

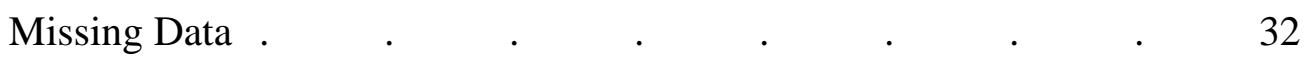

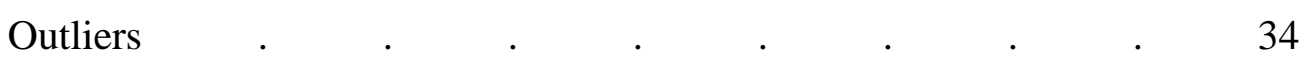

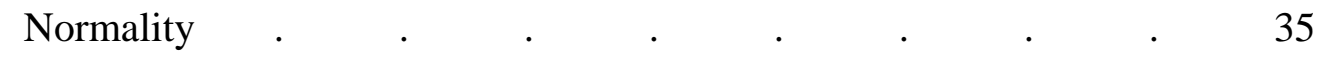

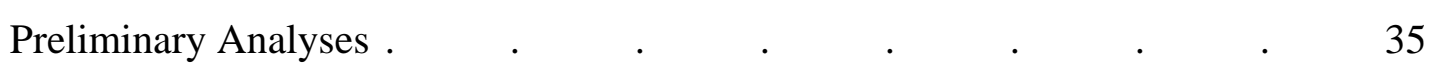

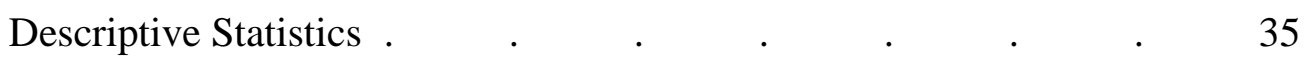

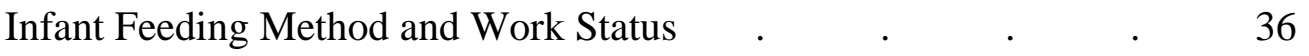

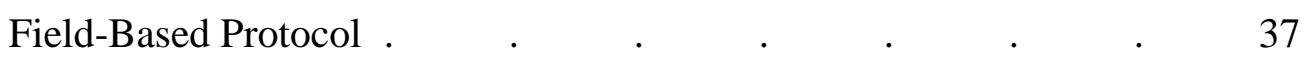

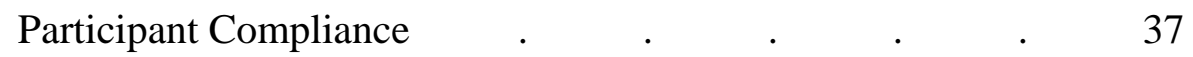

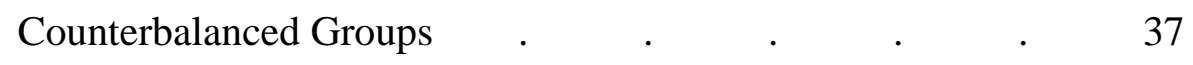

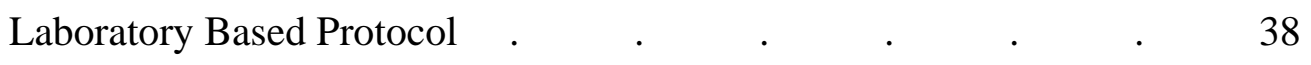

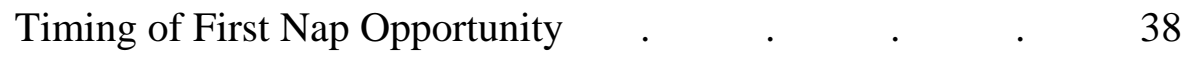

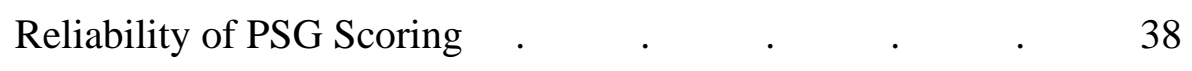

Previous Night Sleep and MSLT Results • 39 
Sleep Disorder Symptoms and MSLT Results _ . . $\quad$. 40

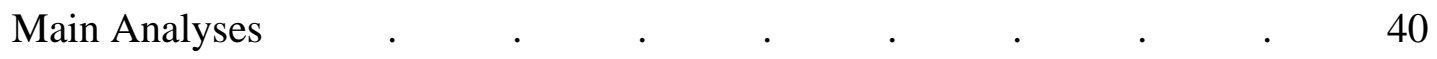

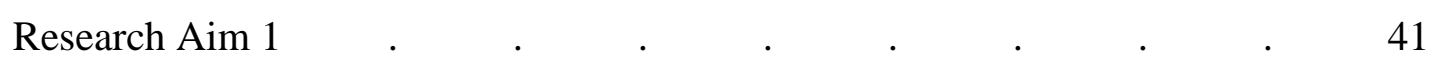

$\begin{array}{lllllll}\text { Research Question 1: Main Analyses . } & \text {. } & \text {. } & \text {. } & \text {. } & 41\end{array}$

Research Question 1: Supplemental Analyses 41

$\begin{array}{lllllll}\text { Research Question 2: Main Analyses . } \quad ~ & \text {. } & \text {. } & \text {. } & \text {. } & 41\end{array}$

Research Question 2: Supplemental Analyses _ 42

Research Question 3: Main Analysis . $\quad$. $\quad$. $\quad$. $\quad$. 42

Research Question 3: Supplemental Analyses _ . 43

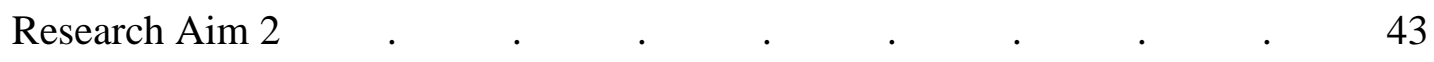

Research Question 4: Main Analyses . $\quad$. $\quad$. $\quad$. $\quad$. 43

Research question 4: Supplemental Analyses. $\quad$ 44

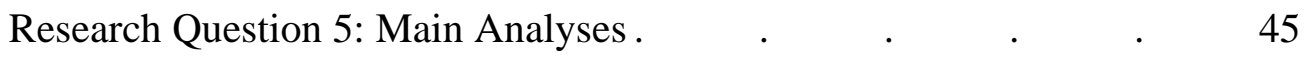

Research Question 5: Supplemental Analyses 45

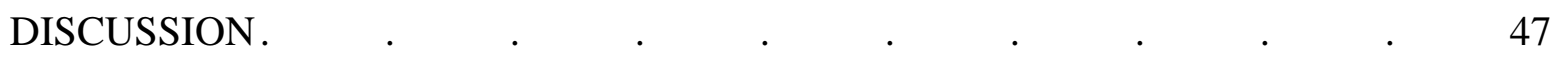

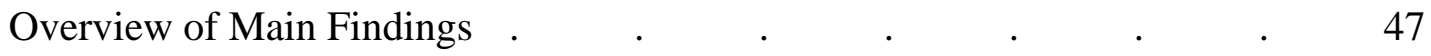

Aim 1: Objective Sleep and Sleepiness $\quad . \quad$. $\quad . \quad$. $\quad . \quad 47$

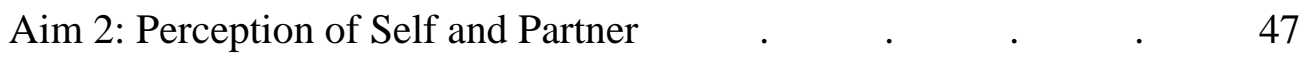

Review and Interpretation of Study Findings: Aim 1. $\quad . \quad$. $\quad$. 47

Research Question 1: Difference in Sleep Indices
Among Mothers and Fathers . $\quad . \quad$.

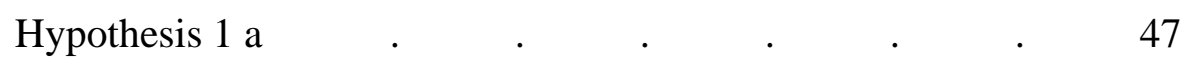

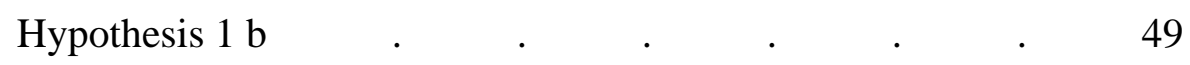

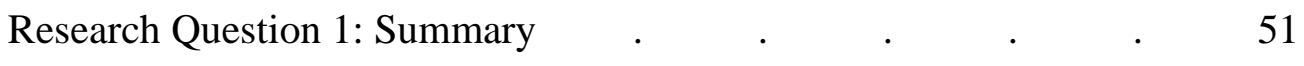


Research Question 2: Sleep and Prediction of Sleepiness $\quad$. $\quad$. 51

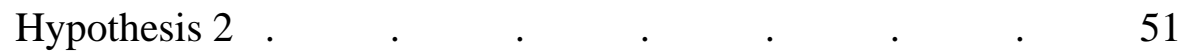

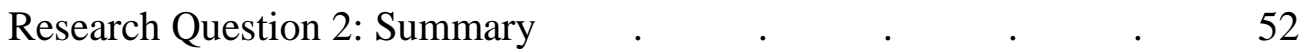

Research Question 3: Sleepiness Levels . $\quad$ • $\quad$. $\quad$. 52

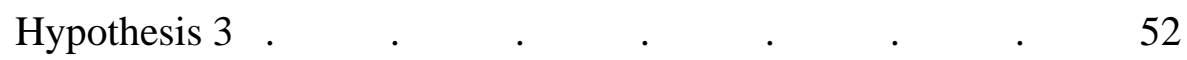

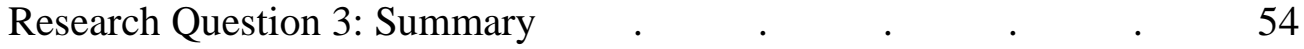

Review and Interpretation of Study Findings: Aim 2. $\quad$. $\quad$ • 55

Research Question 4: Accuracy in Report of Sleepiness 55

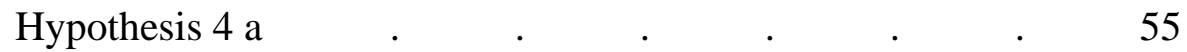

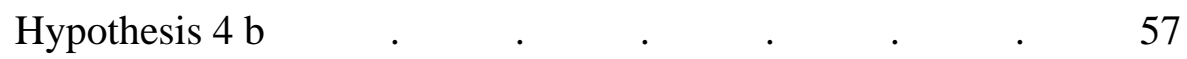

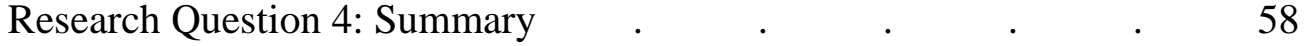

Research Question 5: Accuracy in Report of Nocturnal Wake 58

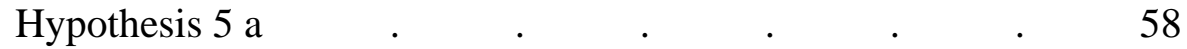

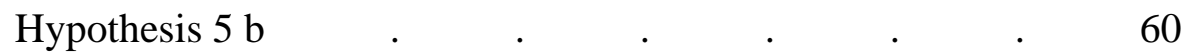

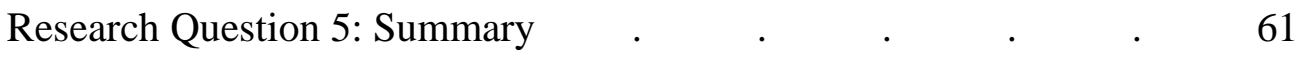

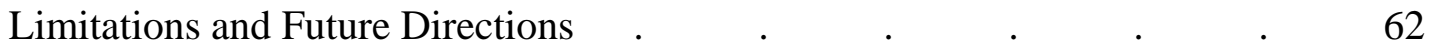

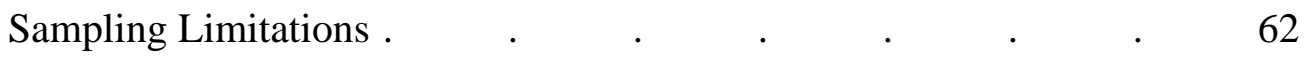

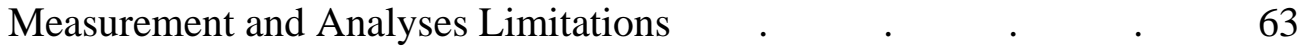

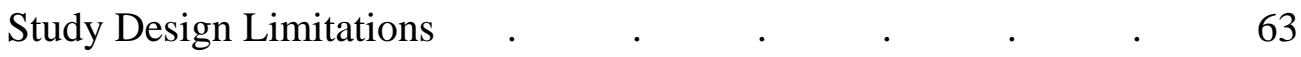

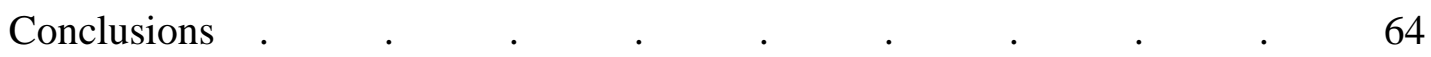

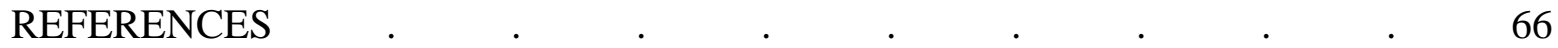




\section{LIST OF TABLES}

1. Pilot Data: Descriptive Statistics for Primiparious Mothers Sleep Variables during postpartum_week three $\quad . \quad$. $\quad . \quad$. $\quad$. 78

2. Sample Characteristics for Total Sample, Mothers, and Fathers _ 79

3. Target sample relationship history: In years $(\mathrm{N}=21) \quad$. $\quad$. $\quad$. 81

4. $\quad$ Constructs in study and respective instruments used to measure constructs . $\quad 82$

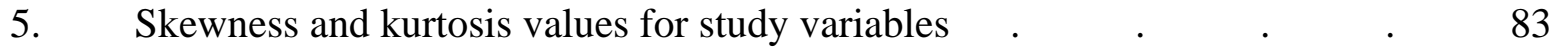

6. Descriptive statistics for, and difference statistics among, mothers'

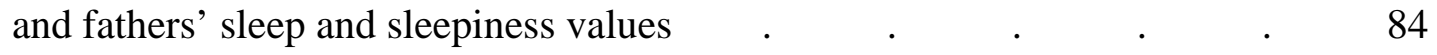

7. Difference among sleep variables from week prior, and night before MSLT: Correlations among differences and sleep onset latency 85

8. Descriptive statistics for self-reported sleep disorder symptoms

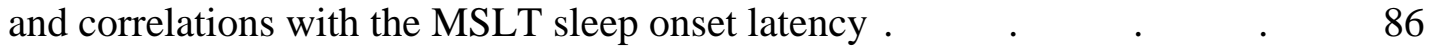

9. Descriptive statistics for self report and report of partner on sleepiness and time awake: differences with objective measures 


\section{LIST OF FIGURES}

1. Timeline for field and laboratory based procedures . $\quad . \quad$. $\quad$. 89

2a. Sleep onset latency for individual mother participants: normative, moderate, and extreme sleepiness ranges identified

2b. Sleep onset latency for individual father participants: normative, moderate, and extreme sleepiness ranges identified

3a. Bland-Altman plots for mothers' self-reports of sleepiness compared to mothers' objectively measured sleepiness: arbitrary guide lines at 0,25 , and 50 minute differences

3b. Bland-Altman plots for fathers' self-reports of sleepiness compared to fathers' objectively measured sleepiness: arbitrary guide lines at 0,25 ,

3c. Plots for mothers' and fathers' self-reports of sleepiness compared to their objectively measured sleepiness: Means differences and standard deviations

4a. Bland-Altman plots for mothers' reports on fathers' sleepiness when compared to father's objectively measured sleepiness: arbitrary guide lines at $0,2.5$, and 5 minute differences

4b. Bland-Altman plots for fathers' reports on mothers' sleepiness when compared to mothers' objectively measured sleepiness: arbitrary guide lines at $0,2.5$, and 5 minute differences

4c. Plots for mothers' and fathers' reports on partners' sleepiness compared to their partners' objectively measured sleepiness: Means differences and

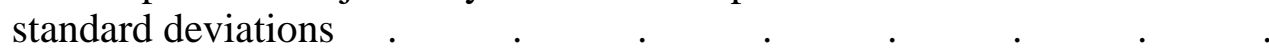

5a. Bland-Altman plots for mothers' self-reports of nocturnal wake time compared to mothers' objectively measured nocturnal wake time: arbitrary guide lines at 0,25 , and 50 minute difference

5b. Bland-Altman plots for fathers' self-reports of nocturnal wake time compared to fathers' objectively measured nocturnal wake time: arbitrary guide lines at 0,25 , and 50 minute differences

5c. Plots for mothers' and fathers' reports on partners' nocturnal wake time compared to their partners' objectively measured nocturnal wake time: Means differences and standard deviations 
6a. Bland-Altman plots for mothers' reports on fathers' nocturnal wake time when compared to fathers' objectively measured nocturnal wake time: arbitrary guide lines at 0,20 , and 40 minute differences _ . 101

6b. Bland-Altman plots for fathers' reports on mothers' nocturnal wake time when compared to mothers' objectively measured nocturnal wake time: arbitrary guide lines at 0,20 , and 40 minute differences

6c. Plots for mothers' and fathers' reports on partners' nocturnal wake time compared to their partners' objectively measured nocturnal wake time: Means differences and standard deviations 


\section{LIST OF APPENDICES}

1. Community Resources for Mental Health and Sleep · _ 104

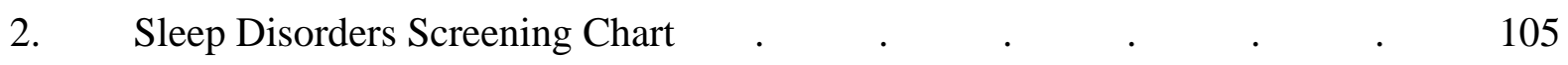

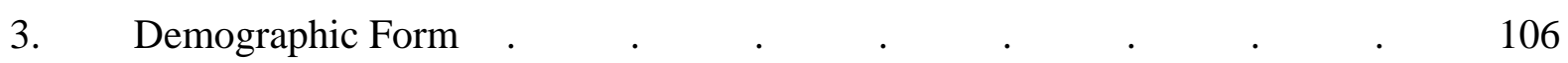

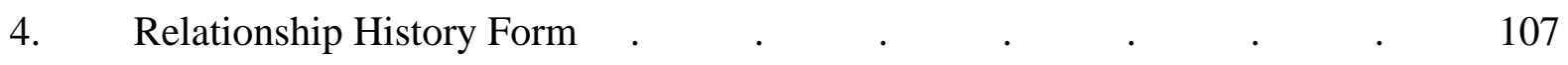

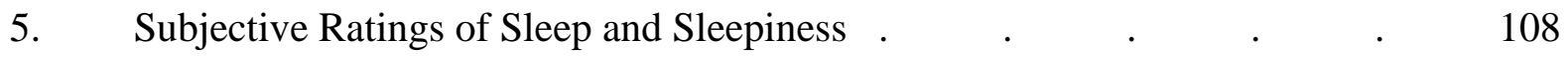

6. Subjective report of Nocturnal Waking $\quad . \quad$. $\quad . \quad$. $\quad$. $\quad 109$

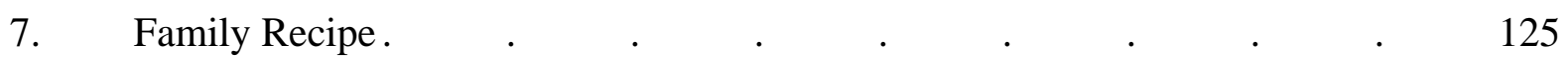




\section{Introduction}

The purpose of the current study was to examine sleep and sleepiness among first-time mothers and fathers during the early postpartum period, and to identify their perceptions of their own and their partner's sleep. A unique aspect of the current study was that it included the assessment of sleep among fathers, who are underrepresented in the postpartum sleep literature. This study was an initial step toward understanding sleep disturbance within the postpartum family.

Within the following section sleep throughout and specifically during the early postpartum period is discussed and is followed by a review of the effects of sleep disturbance. Next, background information on the measures that were used to assess sleep and sleepiness are described. Finally, the current research aims, research questions, and hypotheses are provided.

\section{Sleep Throughout the Postpartum Period}

The human need for sleep and a review of maternal and paternal sleep throughout the postpartum period will be described. The literature that describes possible reasons for sleep disturbance among postpartum parents and the effects that sleep disturbance may have on both caregiving and the relationship between the parents will also be reviewed.

\section{Human Need for Sleep}

Individual needs for sleep are variable and depend on multiple interacting factors that include, but are not limited to, age, health, and daily activity. The typical adult requires approximately 7-8 hours of sleep each night (Ferrara \& De Gennaro, 2001). Ultimately, the amount of sleep recommended for an individual is the amount that allows him or her to feel fully rested upon awakening; this amount of sleep is not the same for everyone. 
Chronic deviation from one's needed duration of sleep may have adverse health and neurobehavioral consequences. Alverez and Ayas (2004) conclude that chronic short sleep duration (i.e. less than the suggested 7-8 hours) leads to long-term health consequences and is also associated with all-cause mortality. According to a dose-response study on the effects of sleep deprivation, Van Dongen and colleagues determined that most people need an average of 8 hours and 10 minutes of objectively measured sleep per 24-hour period to avoid cumulative neurobehavioral deficits on cognitive performance tasks (Van Dongen, Maislin, Mullington, \& Dinges, 2003). That is, as sleep time less than an average of 8 hours and 10 minutes accumulates and becomes what is known as "sleep debt" (Dement \& Vaughan, 1999), neurobehavioral deficits increase.

Sleep deprivation occurs in different forms: total sleep deprivation, partial sleep deprivation, and sleep fragmentation. The most common forms of sleep deprivation during the postpartum period are partial sleep deprivation (when the full amount of the daily sleep need is not acquired, but some is) and sleep fragmentation (brief periodic arousals during sleep) (Drummond \& Orff, 2005). During the postpartum period these two forms of sleep deprivation commonly co-occur; as sleep fragmentation accumulates it reduces sleep time thus leading to partial sleep deprivation. However, sleep fragmentation can also occur without decreasing total sleep time; that is, one could acquire an extended sleep period to counter the wake time promoted by sleep fragmentation. The current study was designed to examine both sleep fragmentation and sleep deprivation among postpartum mothers and fathers.

\section{Postpartum Mothers \& Fathers}

A growing body of literature has examined the sleep of mothers throughout pregnancy and the postpartum period, yet little is known about the sleep of new fathers. The following 
literature review provides a rationale for studying both parents, and for studying them early in the postpartum period.

As reviewed by Ross and colleagues, maternal sleep worsens from the first trimester to the third trimester of pregnancy and becomes most disturbed following delivery (Ross, Murray, \& Steiner, 2005). Kang and colleagues conducted a longitudinal assessment of sleep among mothers from five weeks before delivery through 15 weeks postpartum and found the highest levels of sleep deprivation during the initial postpartum weeks (Kang, Matsumoto, Shinkoda, Mishima, \& Seo, 2002). The highest incidences of sleep fragmentation are reported to occur during the second week postpartum, after which sleep begins to improve for reasons discussed below (Shinkoda, Matsumoto, \& Park, 1999). These authors speculate that during the second postpartum week the infant feeding and sleep-wake cycles begin to synchronize with the variation of day and night. In a 2005 review, Gaylor and Manber add that maternal sleep during the postpartum period is impeded by sleep fragmentation and while this fragmentation gradually decreases across the first 12 months postpartum, the most pronounced decrease is across 6 to 12 months postpartum.

As previously stated, relatively few researchers have explored sleep among postpartum fathers. However, investigations do describe changes in postpartum fathers' sleep from the prenatal to the postpartum period. In a qualitative study, first-time fathers reported that all daily activities center around the infant's caretaking needs and sleeping times (Premberg, Hellstrom, \& Berg, 2008). Furthermore, the sleep time of first-time fathers was significantly lower during the early postpartum period when compared to their sleep time assessed when their partners were at 23 weeks gestation (Condone, Boyce, \& Corkindale, 2004). 
Gay and colleagues collected descriptive sleep pattern and fatigue data from first-time mothers and fathers and found that both parents had less sleep, had more self-reported sleep disturbance, and had higher ratings of fatigue during the first month postpartum when compared to their levels during the pregnancy (Gay, Lee, \& Lee, 2004). Interestingly, fathers had less 24hour sleep than mothers during both pregnancy and the postpartum period; however, mothers had more wake after sleep onset — an assessment of sleep fragmentation — than fathers during both times of assessment. Overall, postpartum parents have more sleep fragmentation and less total sleep time during the initial postpartum period when compared to their levels during the mother's third trimester, and there may be important differences between the sleep of new mothers and fathers. Thus far, no investigations have been conducted to objectively examine sleepiness among postpartum mothers or fathers; the proposed study will contribute to filling this gap. Mechanisms for Disturbed Sleep

Postpartum Physiological Alterations. Sleep among postpartum mothers may be affected by physiological alterations due to pregnancy and childbirth. In a study where mothers slept away from their infants, maternal sleep disruption continued (Karacan, Williams, Hursch, McCaulley, \& Heine, 1969), which may have been due to physiological factors such as various hormonal regulations (Manber \& Armitage, 1999). Physiologic changes that occur during pregnancy may have significant influences on sleep after birth. For example, throughout pregnancy cortisol, estrogen, and progesterone levels increase: high cortisol is associated with stress and depressive symptoms; high estrogen is associated with less REM sleep, fewer night wakings, longer sleep times, and a decrease in sleep latency; and progesterone has a sedative effect and is known to increase non-REM sleep (Manber \& Armitage, 1999). 
The sleep promoting effects of estrogen and progesterone are highest during late pregnancy and decrease immediately after delivery (Manber \& Armitage, 1999; Ross et al., 2005). From a homeostatic viewpoint, the precipitous decrease in these hormones after delivery challenges the body to make up for the absence of their sleep promoting effects during the postpartum period. Although new fathers likely do not have significant sleep disturbing hormonal changes following the birth of their child, the sleep of new fathers may be affected by the disturbed sleep among new mothers that results from postpartum hormonal changes.

Infant Night Waking. Infant night waking contributes to the high prevalence of postpartum sleep disturbance (Santiago, Nolledo, Kinzler, \& Santiago, 2001). Parent sleep is commonly interrupted to respond to the infant's needs. Infant sleep cycles (60 minutes) are shorter than those of adults (70-120 minutes). Similar to adults, infants generally awaken at the end of each sleep cycle. Upon awakening they either signal for the caregiver (cry and wake the caregiver for intervention), or self-soothe (return to sleep without caregiver intervention). Naturally, infant signaling interrupts and thus fragments the sleep of the caregiver (Nishihara, Horiuchi, Eto, \& Uchida, 2002). This signaling is not without purpose. Infants may signal to their parents because they have nutritional needs during the night. High metabolic demands of young infants may necessitate a high frequency of infant feeding, which was found to contribute to maternal sleep disturbance (Thomas \& Foreman, 2005).

Caregiving and Mother / Father Relationships. When infants signal, typically mothers and / or fathers have caregiving responsibilities to carry out. A non-empirical study reported by the $\mathrm{BBC}$ describes that when babies cry in the middle of the night, $52 \%$ of fathers continue to sleep, or pretend to do so ("Dads 'sleep on'," 2004). Along this line, a study of infant childcare and infant interaction among first-time postpartum mothers and fathers revealed that when both 
parents were available, mothers spent more time providing caregiving than fathers (Laflamme, Pomerleau, \& Malcuit, 2002). Conversely, Fagerskiold (2008) conducted a qualitative assessment of the lives of first-time fathers and found that when both parents were home, fathers and mothers equally contributed to infant caregiving. As a caveat, the previous study should be interpreted with caution; the research was conducted among a Scandinavian sample, which may be more collectivist in nature than the American sample that was used in the current study. When parental employment is considered, mothers provide a majority of nocturnal caregiving when they are not working; however, fathers increased their nocturnal caregiving behaviors when mothers returned to work (Jones \& Heermann, 1992). The extent to which either the mother or father carries out the majority of the nocturnal caregiving responsibilities was collected via the current protocol; however, the sleep and the effects of sleep disturbance among new parents was the major purpose of the current study.

New parents' functioning and the relationship between new parents can be directly affected by both their own as well as their partner's sleep disturbance. Maternal sleep disturbance has been found to affect fathers' sleep disturbance, and the converse has also been found (Meijer \& van den Wittenboer, 2007). An examination of first-time parents during the third trimester of pregnancy and postpartum month four revealed that fatigue levels were significantly higher during the postpartum period and did not differ between mothers and fathers (Elek, Hudson, \& Fleck, 2002). In their review of the parenting literature, Nystrom and Ohrling (2003) describe new mothers' and fathers' experience as, “living in a new and overwhelming world" (p. 319). Across the first postpartum year both first-time mothers and fathers report a decrease in marital satisfaction, but an increase in their infant care self-efficacy (Elek, Hudson, \& Bouffard, 2003; Meijer \& van den Wittenboer, 2007). 
Sleep deprivation is known to have a multitude of adverse effects on health and functioning (described below), and during the postpartum period both parents are highly susceptible to sleep deprivation. Early in the postpartum period - when sleep is most disturbed many new mothers and fathers return to work and are expected to continue being productive members of society. According to the Family Medical Leave Act (FMLA) parents are allowed 12 weeks of unpaid family leave for medical conditions that include care for a newborn, if they are employed by a company with more than 50 employees (United States Department of Labor, 1993). According to the A Better Balance Legal Team, a legal group fighting for flexibility in the workforce for new parents, "Lower-income workers, even if eligible, cannot afford to take unpaid leave.” (A Better Balance, 2010, p. Family Leave). Furthermore, the United States is the only industrialized country that does not have a policy for paid, job-protected maternity and paternity leave (Kamerman, 2000). For a review of the impacts that the current FMLA has on family structure, functioning, and infant outcomes see (Schor, 2003).

New parents may have a difficult time balancing their roles as involved parents in infant caregiving as well as contributors to their family income. The current FMLA policy is incongruent with the knowledge about positive outcomes of longer maternity and paternity leaves. Among mothers, longer maternity leave is associated with better maternal mental health, increased breast feeding, and better infant health outcomes (Staehelin, Bertea, \& Stutz, 2007). Among fathers, an active parental role is important because the time that fathers spend with their infants following birth leads to positive infant and child outcomes (Cabrera, Tamis-LeMonda, Bradley, Hofferth, \& Lamb, 2000). Furthermore, when fathers take longer periods of family leave they are more involved in infant caregiving nine months later (Nepomnyaschy \& Waldfogel, 2007). 


\section{Effects of Sleep Disturbance}

Sleep disturbance has a multitude of deleterious effects on functioning including hormone regulation (Copinschi, 2005), general health (Alvarez \& Ayas, 2004), decision making (Harrison \& Horne, 2000), and performance (Durmer \& Dinges, 2005). For the purpose of the current review, discussion of these effects will be limited to parental mood, infant wellbeing, and parental daytime sleepiness.

\section{Parental Mood}

The association between sleep and mood disturbance is well established: experimentally induced partial sleep deprivation has negative effects on mood. Participants who were restricted to 4-5 hours of sleep per night for 7 consecutive nights demonstrated worsened scores on the Profile of Mood States total mood disturbance, and specifically on subscales for fatigue-inertia, confusion-bewilderment, and tension-anxiety (Dinges et al., 1997). Bonnet (1985) demonstrated that two nights of experimentally induced sleep fragmentation degraded mood and performance to levels lower than those found after one night of total sleep deprivation. The findings were of particular interest because participants only lost approximately one hour of sleep per night as a result of induced sleep fragmentation.

Poor postpartum sleep is associated with heightened levels of mood disturbance among new parents. In an assessment of infant behaviors and parental well-being, Smart and Hiscock (2007) found that parents were concerned with their infant's sleep problems and infant sleep problems were associated with poor parental mental health.

Postpartum depression among parents is well documented (Paulson, Dauber, \& Leiferman, 2006). Across the postpartum period up to $85 \%$ of women report having experienced an increase in negative mood and 10-15\% reach the diagnostic criteria for depression (Ross et 
al., 2005). Overall, more sleep loss among postpartum women appears to be associated with increased dysphoric mood states (Swain et al., 1997). More specifically, among postpartum women higher levels of sleep fragmentation are associated with more negative mood states (Lee, McEnany, \& Zaffke, 2000). The effects of sleep disturbance on mood are so pronounced that Armstrong and colleagues have suggested that postpartum women who suffer from chronic sleep disturbance are over-diagnosed with depression because of the similarities in affect due to sleep disturbance and postpartum depression (Armstrong, Van Haeringen, Dadds, \& Cash, 1998).

Although disentangling the causes and effects of postpartum depression is a challenge in the field of sleep, there is evidence that parentally reported "infant sleep problems" (e.g. extensive night waking and difficulty going to sleep) contribute to the onset of maternal depression (Dennis \& Ross, 2005; Hiscock \& Wake, 2001; Hiscock \& Wake, 2002). Additional research indicates that mothers with depressive symptoms are less likely to endorse healthy infant sleep practices (e.g. regular bedtime routines, environments conducive to sleep) than mothers without depressive symptoms (Paulson, Dauber, \& Leiferman, 2006). Depressive symptoms among postpartum mothers were found to predict a higher frequency and duration of infant night waking (Warren, Howe, Simmens, \& Dahl, 2006). Karraker and Young (2007) describe the direction of effects between symptoms of postpartum depression and infant sleep as transactional. That is, simultaneously, infant night waking increases symptoms of maternal depression, and symptoms of maternal depression increases less healthy infant sleep practices, which may ultimately promote infant night waking.

The literature on sleep and postpartum depression among new fathers is less developed than that among new mothers. However, steps have been made to include fathers in the assessment of postpartum depression. For example, the Edinburgh Postnatal Depression Scale 
has recently been validated for men (Matthey, Barnett, Kayanagh, \& Howie, 2001). Furthermore, there appears to be a significant correlation between the incidence of depression symptoms among mother and father couples (Soliday, McCluskey-Fawcett, \& O’Brien, 1999). Smart and Hiscock (2007) performed the first assessment of infant sleep and depression among new fathers; fathers who reported that their infants had sleep problems also reported higher levels of depressive symptoms. Furthermore, these researchers call for a more thorough assessment of depression among new fathers especially due to the current increase in paternal caregiving (Lamb \& Bornstein, 2002).

\section{Infant Wellbeing}

Although the current study did not focus directly on the infant, the effects of parental depression on the infant is reviewed in order to demonstrate that there may be direct, as well as indirect effects of parent sleep on infant wellbeing. Depression associated with sleep disturbance may result in compromised infant outcomes. As reviewed by Murray and colleagues, postnatal depression is strongly associated with an increase in negative infant-parent interactions and adverse emotional and cognitive outcomes for the infant (Murray, Cooper, \& Hipwell, 2003). A large national study revealed that parents with depressive symptoms are 1.5 times more likely to engage in more negative parent-infant interactions than parents without depressive symptoms (Paulson et al., 2006). More specifically, infants of mothers suffering from postpartum depression have been shown to have more eating and sleeping difficulties, as well as fewer vocal, visual, and physical interactions with their mother (Righetti-Veltema, Conne-Perreard, Bousquet, \& Manzano, 2002). Additionally, even among non-depressed mothers at 3 months, fatigue was associated with difficulties in maternal-infant relationships. In sum, postpartum sleep disturbance is important to examine because of its close association with infant wellbeing. 


\section{Excessive Sleepiness}

Excessive daytime sleepiness is a symptom of sleep disturbance and is the propensity to fall asleep during a time when one intends to be awake (Benbadis, 2005). Excessive daytime sleepiness can be caused by a variety of factors that do not allow acquisition of an appropriate amount of sleep. These factors cover a vast range that include self-imposed insufficient sleep, sleep disorders, and various intrinsic and extrinsic factors that disturb sleep. The magnitude of excessive sleepiness occurs in grades: the greater the magnitude of sleepiness, the more likely one is to fall asleep during waking activities such as sitting quietly, or (too commonly) driving a car (Vaughn \& D'Cruz, 2005). These authors note that as sleep disturbance - and thus daytime sleepiness - accumulates, people become worse judges of their own levels of sleepiness because they become accustomed to their condition. Their inability to accurately perceive their sleepiness is particularly poor when excessive sleepiness becomes persistent across time, as it may become for parents during the postpartum period.

High profile disasters caused or influenced by excessive sleepiness include the Challenger space shuttle explosion, the Exxon Valdez oil spill, and the Chernobyl and Three Mile Island nuclear power plant meltdowns (Walsh, Dement, \& Dinges, 2005). According to the 2002 Gallup survey $37 \%$ of drivers have reported nodding off or falling asleep while driving a car and roughly 56,000 car crashes annually are attributed to drowsy driving. However, according to experts, statistics that estimate accidents associated with sleepiness are grossly underestimated because there is no way to objectively test for sleepiness in the field (Dinges, 2005).

Daytime sleepiness and its effects have been extensively studied in high-risk groups including medical residents, airline pilots, commercial drivers, and shift workers. Postpartum 
parents, and especially fathers, remain a relatively understudied population. Yet new parents drive, are responsible for the care and safety of their newborn infant, and are expected to return to work shortly after the birth of their child. Postpartum women demonstrate high levels of impaired functioning when examined with the psychomotor vigilance test (Insana \& Montgomery-Downs, 2007); however, an objective measure of sleepiness among postpartum mothers or fathers has yet to be catalogued.

\section{Overview of Objective Sleep Measurement Methods}

Objective measures for assessing postpartum sleep are rarely used, and there has been a call for greater use of them (Ross et al., 2005). The protocol described below branches out from a larger study for which actigraphy - an objective measurement technique - is used to assess sleep among postpartum women. For the current study actigraphy was used to measure sleep, in addition to polysomnography and the Multiple Sleep Latency Test to measure sleepiness. Each of these techniques is briefly reviewed before their detailed description in the methods section to follow.

\section{Actigraphy}

Actigraphy (activity-based monitoring) uses general body movements to identify periods of sleep and wakefulness. An actigraph is a small motion sensor worn like a watch on one's nondominant wrist. Actigraphs are capable of collecting activity data over relatively prolonged periods and use computer-based algorithms to estimate sleep and wake states from activity values. Actigraphy is a reliable instrument for measurement of sleep in adults (Jean-Louis et al., 1997; Kushida et al., 2001; Sadeh \& Acebo, 2002). A recent validation study among adults compared actigraphy to the 'gold standard,' polysomnography (PSG), and found significant agreement between the two techniques (de Souza et al., 2003). 
Due to the use of general body movements to detect sleep, periods of immobility are identified as sleep bouts. Thus, actigraphy is vulnerable to artifact such as when the actigraph is taken off and is motionless; such a period could be misidentified as sleep. Therefore, it is essential that concurrent sleep diaries be maintained in order to behaviorally corroborate actigraphy data and to eliminate artifact (Acebo \& LeBourgeois, 2006).

\section{Polysomnography}

PSG literally translates to "many sleep writings." These measures have been used in sleep research beginning in the 1950 's by Nathanial Kleitman and colleagues. Since that time PSG has become an important sleep assessment tool. Typically PSG measurements for sleep assessment are acquired from electroencephalography, which measures electrical potentials from general brain regions; electrooculography, which measures eye movements; and submental electromyography, which measures muscle tone. The combination of these measurements from multiple channels of physiological data, along with direct observation via real time in-room video transmission, allow an observer to differentiate wakefulness from the different stages of sleep which are scored in 30-second epochs. Standard criteria for classifying the different stages of sleep are described in the American Academy of Sleep Medicine Manual for the Scoring of Sleep and Associated Events (2007). PSG is the electrophysiological measurement instrument used to administer the Multiple Sleep Latency Test. Multiple Sleep Latency Test

The laboratory-based Multiple Sleep Latency Test (MSLT) is a test developed in the 1970’s (Carskadon \& Dement, 1975) to objectively index sleepiness (Afifi, Kushida, \& Carskadon, 2005). Since the initial development of the MSLT, the test has gone through various stages of standardization (Carskadon et al., 1986; Thorpy et al., 1992); practice parameters are 
described by the American Academy of Sleep Medicine (AASM) (Littner et al., 2005). Practice parameters are necessary to ensure standard use of the MSLT (Carskadon et al., 1986). The MSLT is considered the 'gold standard' measurement of sleepiness and has been used to index levels of sleepiness among various populations and due to many diverse sleep disorders and other causes of sleeplessness (Arand et al., 2005; Littner et al., 2005). However, the MSLT has never been used to explore sleepiness among postpartum parents. The MSLT demonstrates a test-retest reliability of .97 within a normative sample (Littner et al., 2005). The validity of the MSLT is fundamentally difficult to determine because the MSLT is considered the 'gold standard' measure of sleepiness; however, the face validity of the MSLT is considered high (Afifi et al., 2005; Arand et al., 2005; Littner et al., 2005).

Briefly, the standardized MSLT protocol requires that participants have their sleep assessed for one to two weeks prior to the test via actigraphy or sleep/wake diaries. At no time during the test are participants allowed to consume alcohol or caffeine. The MSLT begins from 90 to 180 minutes after the usual morning awakening. At this time participants have PSG sensors applied, then they are provided with a nap opportunity in the lab, by which they are instructed to allow themselves to fall asleep. The time that it takes for participants to initiate sleep is called sleep onset latency and is identified by standard PSG scoring criteria. The test consists of four to five of these nap opportunities conducted at 2-hour intervals - measured from the beginning of one nap to the beginning of the next). The average sleep onset latency from these sessions is the outcome variable of this test. Normative data derived from a meta-analysis of control groups described as normal sleepers - from various studies that utilized the MSLT indicate that typical sleep onset latency is $10.4(S D=4.3)$ minutes for the four-nap MSLT (Littner et al., 2005). As reviewed by Afifi and colleagues (2005), daily average sleep onset latency scores $\leq$ five minutes 
indicate a pathological level of daytime sleepiness, $>$ five to ten minutes indicate a moderate sleepiness, and $>$ ten minutes indicate more normative levels of sleepiness.

\section{Statement of the Problem}

As previously stated, the purpose of the current study was to examine sleep and sleepiness among first-time mothers and fathers during their early postpartum period. Additionally, the current study identified the perceptions of postpartum parents regarding their own sleep and their partner's sleep.

The sleep of new mothers is known to worsen throughout pregnancy and becomes most disturbed in the early postpartum period. Researchers indicate that mothers' sleep-wake cycles are most irregular - with increased waking at night - from pregnancy through roughly the fifth postpartum week (Gay et al., 2004; Shinkoda et al., 1999). The current study examined sleep and functioning among first-time mothers and fathers during their early postpartum period when maternal sleep disturbance is highest.

Little research has been performed to examine sleep among postpartum fathers. Yet new fathers are presumably susceptible to sleep deprivation from infant signaling during the night and the contribution that fathers may provide towards nocturnal caregiving responsibilities. Furthermore, fathers are typically expected to return to work almost immediately after the birth of their child even though they may experience the effects of disturbed sleep - which is known to greatly influence transportation and workplace accidents (Walsh et al., 2005). By extending research to understand sleep and sleepiness among postpartum fathers, steps can be made to promote safety and wellbeing among new fathers as well as to promote the health of the family as a whole. 
Researchers call for an understanding of the effects of disturbed sleep and sleep loss among new parents especially with the use of objective measurement tools (Gay, et al., 2004; Ross et al., 2005). The current study was the first to include an objective examination of the levels of sleepiness among postpartum mothers and fathers. Additionally, the current study was the first to examine first-time parents' perceptions of each other's sleeping behaviors and sleepiness. The perceptions of new mothers and fathers were examined in order to provide an understanding of self-report and partner perception during the early postpartum period when their sleep disturbance is high. By examining both first-time mothers and fathers early in their postpartum period, the framework is established to better understand the function and dysfunction of new families.

\section{Pilot Data}

Descriptive statistics were calculated on a sample of primiparious mothers $(N=17)$ from a larger study to demonstrate the variability in demographics among the local population, as well as their typical sleep profile during postpartum week three. Mothers were $25.8(S D=4.28$, range 21 - 35) years old, $94.1 \%$ were white, had a mean income of $\$ 51,462(S D=\$ 39,278$, range $\$ 5,000-\$ 150,000)$, and had $15.9(S D=2.8$, range 12 - 22) years of education. Of these women, 82.4\% lived with their partner, $76.5 \%$ fed their infants exclusively with breast milk, and $41.2 \%$ napped at least once during postpartum week 3 . However, the overall frequency of naps was low; across all participants, there were a total of 19 naps during a collective 119 days. Descriptive statistics for postpartum week three sleep data can be seen in Table 1.

\section{Research Questions (RQ) and Hypotheses (H)}

The aims of this dissertation study were to: 1.) objectively examine the difference in sleep and sleepiness levels among first-time mothers and fathers during their early postpartum 
period; 2) examine parents' perceptions of their own and their partner's sleep, and sleepiness. The following research questions and hypotheses correspond to the aims of the study. Each research question is preceded by a rationale, and followed by corresponding hypotheses.

Aim 1: Research Questions for Objective Sleep and Sleepiness Data

RQ1: Rationale

Previous research indicates that mothers had less nocturnal sleep but more 24-hour sleep than fathers; that is, mothers took daytime naps to inflate their 24-hour sleep time to levels higher than fathers (Gay et al., 2004). However, previous research also indicates that many mothers with young infants do not frequently nap (Cottrell \& Karraker, 2002). Additionally, the pilot data calculated for the current study, as described above, revealed that our laboratory's sample of first-time postpartum women nap very little. Therefore, the expected sample of postpartum women for the current project was expected to have less nocturnal sleep (as indicated by previous research), yet would not take extra nap opportunities (as indicated by previously described pilot work).

RQ1: How do sleep indices differ between new mothers and fathers during the first postpartum month?

H1 a: Postpartum mothers will have less 24-hour sleep time than postpartum fathers. H1 b: Postpartum mothers will have more nocturnal sleep fragmentation than postpartum fathers.

\section{RQ2: Rationale}

Objective sleep indices derived via actigraphy were expected to predict objective sleepiness levels derived via the MSLT in postpartum mothers and fathers. This research question does not specify a-priori which actigraphy sleep index will more strongly predict 
sleepiness because this research question is exploratory. Previous research indicates that sleep experimentally induced sleep restriction leads to increases in objective and subjective sleepiness (Carskadon \& Dement, 1981). Thus far no research has indicated what sleep parameters (i.e. sleep restriction or sleep fragmentation) may have a greater effect on sleepiness among postpartum parents.

RQ2: Will objective sleep indices during the first postpartum month predict mothers' and fathers' subsequent sleepiness?

H2: Total sleep time and sleep fragmentation will predict sleepiness in postpartum mothers and fathers.

RQ3: Rationale

Postpartum mothers were expected to have higher levels of sleepiness than postpartum fathers because mothers were expected to have less total sleep and more sleep fragmentation than fathers. If mothers received less total sleep they would likely demonstrate higher levels of sleepiness than fathers. Furthermore, according to Bonnet's Sleep Continuity Theory, a consolidated interval of sleep is necessary for sleep to be restorative (Bonnet, 1986). Therefore, according to Bonnet's theory, if mothers' sleep was more fragmented then fathers' sleep, mothers would demonstrate higher levels of sleepiness.

RQ3: Will postpartum mothers or fathers have higher levels of sleepiness during the first postpartum month?

H3: Postpartum mothers will have higher levels of sleepiness than postpartum fathers. 


\section{Aim 2: Research Questions for Subjective Sleep Data}

RQ4: Rationale

Postpartum fathers' self and partner reports of sleepiness were expected to be more accurate than mothers' self reports because fathers were expected to have more 24-hour sleep and less sleep fragmentation than mothers. Furthermore, disturbed sleep is known to adversely affect judgment. As sleep disturbance accumulates - and thus daytime sleepiness - people become worse judges of their own levels of sleepiness (Vaughn \& D’Cruz, 2005).

RQ4: Will postpartum mothers or fathers be more accurate in reporting their and their partner's levels of sleepiness?

H4 a: Postpartum fathers' self-reports of sleepiness will be more accurate than postpartum mothers' self-reports.

H4 b: Postpartum fathers will be more accurate than postpartum mothers in reporting their partner's sleepiness.

RQ5: Rationale

Similar to the rationale for RQ4, postpartum fathers' self and partner reports of previous night sleep were expected to be more accurate than mothers' reports because fathers were expected to have less sleep fragmentation, more 24-hour sleep time, and lower levels of sleepiness than mothers. Sleep quality is known to impact daytime functioning and perception of sleepiness (Vaughn \& D’Cruz, 2005). Therefore, mothers were expected to have a greater misperception of their sleep from the previous night compared to fathers.

RQ5: Will postpartum mothers or fathers be more accurate in reporting the amount of time they and their partner were awake the previous night? 
H5 a: Postpartum fathers' self-reports of the amount of time they were awake during the previous night will be more accurate than postpartum mothers' self-reports.

H5 b: Postpartum fathers will be more accurate than postpartum mothers in reporting the amount of time their partner was awake the previous night. 
Method

Participants

\section{Target Sample}

Participants were required to be first-time postpartum parents, from the same mother / father dyad, and to cohabitate. Participants were required to be within three to nine weeks postpartum.

Potential participants were excluded based on the following criteria: pregnancy with multiples, premature delivery, or infant admission to the neonatal intensive care unit.

Additionally, participants were excluded based on a history of major depressive disorder, current treatment for depressive disorder, or a score of $\geq 16$ on the Center for Epidemiologic Studies Depression Scale, a 20-item survey used to determine risk for major depressive disorder (Radloff, 1977). Potential participants with preexisting depression or those who were at risk for major depressive disorder were excluded because participation in the study was considered to be a potential burden on them, and because evaluation of postpartum onset of depressive symptoms was the purpose of a larger study, from which the majority of postpartum couples were expected to be recruited. Potential participants who were ineligible for the study on the basis of their report of a preexisting major depressive disorder, or a score of $\geq 16$ on the Center for Epidemiologic Studies Depression Scale were provided information about community resources for evaluation and treatment (see Appendix 1).

Participants were excluded from taking the MSLT if they foresaw their inability to abstain from using caffeine during the day of the MSLT; the rationale for this exclusion is because caffeine has been found to suppress sleepiness as measured by the MSLT (Lumley, Roehrs, Asker, Zorkck, \& Roth, 1987). 
Mothers and fathers were recruited during their pregnancy or immediately after birth, but prior to their second postpartum month. All participants were recruited via word-of-mouth and via community advertisements, placed to encourage a demographically and socioeconomically representative sample.

Overall, the target number of participants was $N=42(n=21$ mothers and $n=21$ fathers). This target sample size was derived from effect sizes that were calculated based on values reported in previous studies; the studies examined differences between women and men on actigraphy and MSLT sleep outcome variables. Hand calculations were performed to derive sample sizes and were confirmed with G Power Version 2.0 (Faul \& Erdfelder, 1992).

\section{Target power for actigraphy analyses}

Based on an effect size $(d=1.20)$ calculated from a previous study that compared actigraphy sleep fragmentation values between postpartum mothers and fathers (Gay et al., 2004), a sample size of $n=12$ per group $(N=24)$ is required to achieve power $=.80$ with a twotailed alpha level $=.05$ (Denenberg, 1976). Likewise, according to G Power a total sample size of 24 participants ( $n=12$ per group) are calculated based on the values indicated above.

\section{Target power for MSLT analyses}

A sample size of roughly 10 per group is commonly reported in studies that use the MSLT to examine differences between groups in levels of sleepiness (Afifi et al., 2005; Geisler et al., 2006). However, based on an effect size $(d=.90)$ calculated from a previous study that compared MSLT sleep latency values among women and men (Geisler et al., 2006), a sample size of $n=21$ per group $(N=42)$ is required to achieve a power $=.80$ with a two-tailed alpha level $=.05$ (Denenberg, 1976). Likewise, according to G Power a total sample size of 42 participants ( $n=21$ per group) was calculated based on the values indicated above. 


\section{Study Sample}

Twenty-three postpartum couples participated in the study. One couple dropped out of the study because of a death in their family. One couple was dropped from the analyses because they had a 3.5-year-old in addition to their newborn infant. No couples were excluded from taking the MSLT because of their inability to abstain from using caffeine during the day of the MSLT. No data from couples who dropped out of the study were included in any analyses.

The target number of participants was attained $(N=42: n=21$ mothers, and $n=21$ fathers). Participant demographic information is indicated on Table 2 and relationship history is indicated on Table 3 . Precisely $90.48 \%$ of mothers were recruited from and concurrently participated in a field-based NIH funded study that examined postpartum sleep disturbance and its effects on maternal functioning (NIH grant R21HD053836).

\section{Measures}

\section{Actigraphy}

Nonintrusive activity monitoring was recorded with Mini Mitter's Actiwatch-64 (AW64) actigraphs. Accelerometric sensors within these devices interpret motion using digital integration presented as mathematical calculations. The highest resolution setting was used, and allows up to 11 continuous days of monitoring at 15-second epochs. The AW-64 has 64 kbytes of non-volatile memory and is powered by an internal 2030 battery. The AW-64 is $28 \times 27 \times 10$ mm and weighs 16 grams. Actiware ${ }^{\circledR}$ Software Version 5.5 was used to manage, analyze, and archive AW-64 data. Specifications were provided by Mini Mitter Co., Inc., Bend, Oregon.

\section{Personal Digital Assistant (PDA)}

Palm Zire 72 personal digital assistants (PDA) were loaded with software customized for current laboratory studies. The software was developed in consultation with Bruner Consulting, 
Inc. The software includes perception of sleep quality questions, a voice recording function, and sleep and watch diaries. The Palm was maintained using a computerized experience sampling method - a computerized method that permits participants to record momentary behaviors in daily life (Barrett \& Barrett, 2001). Data collected with the software were downloaded and interfaced into an Excel spreadsheet. Measures collected with the Palm software are discussed below.

Perception of sleep quality. Perception of sleep quality was assessed with three questions pertaining to sleep and sleep quality from the previous night derived from the Lee's General Sleep Disturbance Scale (Lee, 1992). Participants were asked (1) how many times they think they woke up, (2) how long they were awake, and (3) to indicate their quality of sleep on a scale of $0-100(0=$ bad and $100=$ good $)$. Participants rated their perception of sleep quality for both themselves and their partner.

Voice note function. Voice recordings were used to identify why participants woke up the previous night. Participants' used the voice recording function to elaborate on the directive, "Describe why you woke up and what you did during each awakening last night."

Sleep and watch diaries. Participants used the diaries to record in real time when they went to bed for the night, when they woke up for the day, when they removed their actigraphs, and when they put their actigraphs back on. The diaries also had a post-hoc recording function that allowed participants to retrospectively record when a sleep or watch event may have happened. The sleep and watch diaries were used to identify sleep periods on the actigraphy recordings described below. 


\section{Sleep/Wake States}

Periods of sleep were identified on the actigraph output with behavioral corroboration by the PDA sleep diary. Sleep onset began at the beginning of two consecutive minutes of no activity on the actigraphy signal that followed the sleep onset time reported in the PDA sleep diary. Conversely, wake was identified after two consecutive minutes of no activity on the actigraphy signal that preceded the wake time reported in the PDA sleep diary. Once periods of 'rest' were identified from the corroboration of these methods, the 'rest' interval was used to calculate total sleep time, nocturnal sleep time, sleep fragmentation indices, sleep efficiency, and nocturnal wake time via Actiware software.

Total sleep time. Total sleep time is the number of minutes scored as sleep during an identified 'rest' interval. Sleep time was calculated by implementing an algorithm that takes into account the activity recorded from four minutes surrounding each 15 -second epoch analyzed. The generated value from this algorithm is then compared to the "Wake Threshold Value" manually set - of 40 . Thus, if activity is $\leq 40$ that 15 -second epoch is scored as sleep, if the activity count is $>40$ that 15 -second epoch is scored as wake. The epochs scored as sleep or wake are then converted into minutes. Total sleep time values for both nocturnal sleep and the preceding naps were added together to form a 24-hour sleep time value. Total sleep time that does not include naps is called 'nocturnal sleep time' and total sleep time that does include naps is called '24-hour sleep time.'

Fragmentation Index. The time elapsed between first epoch and the last epoch scored as sleep within a 'rest' interval is called the 'sleep interval'. The fragmentation index is the amount of activity that occurs between the start time and the end time of the sleep interval. This index is derived from the combination of two calculated percentages. First, the algorithm that calculates 
total sleep time is used to calculate the percentage of epochs scored as movement within an identified nocturnal sleep interval. Second, within a specified sleep interval, an entire minute (four consecutive 15-second epochs) is scored as movement if a single epoch within that minute is scored as movement (activity is $>40$ ); this value is converted into a percent that represents the sleep interval. The two percentages described above are added together to form the fragmentation index. The value of sleep fragmentation did not include naps because sleep fragmentation is activity during sleep, and activity during a nap typically leads to the end of that nap period; furthermore, activity during a nap period is unlikely.

Sleep Efficiency. Sleep efficiency is the amount of total sleep time identified within the 'rest' interval. Sleep efficiency is represented as a percent.

Nocturnal Wake Time. Total wake time is the number of minutes scored as wake during an identified sleep interval. Wake time is calculated by implementing the same algorithm that is used to calculate nocturnal sleep time. The epochs scored as wake that occur during the identified Nocturnal sleep interval are converted into minutes of nocturnal wake. MSLT

An overnight PSG sleep study is typically carried out the night before a MSLT. The purpose of the PSG overnight sleep study is to examine the quality and quantity of sleep on the night prior to the MSLT, and to clinically diagnose potential sleep disorders (Littner et al., 2005). Both prior night's sleep and sleep disorders can affect MSLT results (Afifi, Kushida, \& Carskadon, 2005; Littner et al., 2005). However, for the current research protocol, participants did not have an overnight PSG or a MSLT for a clinical assessment; that is, to identify and treat excessive daytime sleepiness. Instead, the MSLT was used as an instrument to index levels of sleepiness only. Furthermore, an overnight PSG study the night before an MSLT may cause 
delirious effects on sleep, which could intern effect the MSLT results. When participants sleep in a sleep laboratory for the first time, there is typically a 'first-night effect'. The first-night effect is a delayed sleep-onset period and lower sleep efficiency during a first night of sleeping in an unfamiliar environment (i.e. the laboratory); on subsequent nights there does not appear to be as much of a first-night effect (Tamaki, Nittono, Hayashi, \& Hori, 2005). Therefore, more practical methodologies were used to replace the overnight PSG sleep study prior to the MSLT.

Specifically, actigraphy and sleep diaries were used to monitor sleep and wake for one week prior to the MSLT. These data were used to compare the quality and quantity of sleep during the night prior to the MSLT to the average sleep qualities and quantities from the six days prior. Also, a 10-item sleep disorders symptoms screening survey (Thorpy, 2003) was verbally administered to participants by research personnel (Appendix 2). The screening chart was used to examine potential relations among sleep disorder symptoms and subjective and objective sleep and sleepiness measures.

The MSLT was conducted in the sleep research laboratory at West Virginia University. All electrodes were placed according to the standard Ten-Twenty System of Electrode Placement (Carskadon \& Rechtschaffen, 2005). The PSG montage included four channels of EEG (C3 / M2, C4 / M 1, O1 / M 2, and O2 / M 1), EOG (right outer canthus [ROC] / M 1 and left outer canthus [LOC] / M 2), EMG (submentalis), and electrocardiography (ECG). PSG was conducted with the Embla N7000 multichannel polysomnography recording system. All PSG recording channels were plugged into a portable $25 \mathrm{~mm}$ x $130 \mathrm{~mm}$ x $190 \mathrm{~mm}$ Bedside Unit, which is amplified by the system's Communication Unit that is supported by an AC main voltage of 115 $\mathrm{V} / 230 \mathrm{~V}$ and $160 \mathrm{~mA}$ power supply rating. Rembrandt ${ }^{\circledR}$ software was used to manage, analyze, and archive PSG data. 
Once participants were connected to all PSG measures, impedance levels were checked (i.e., the sensitivity of the connections between the scalp and the electrodes that are $<5.0$ on a scale of 100). Appropriate impedance levels assure that an electrode records an accurate electrical potential with minimal noise.

Prior to the first MSLT nap opportunity the research personnel performed biological calibrations; that is, the personnel instructed participants to perform several movements in order to provide baseline readings of the PSG equipment. Biological calibration instructions included: “Close your eyes. Open your eyes. With your head still and your eyes closed look left, look right, look left, and look right. With your head still and your eyes closed look up, look down, look up and look down. Grind your teeth."

The MSLT nap opportunity began with the instruction "Please lie quietly, assume a comfortable position, keep your eyes closed, and try to fall asleep." The instruction is immediately followed with the indication of 'lights out' on the Rembrandt ${ }^{\circledR}$ software. During the nap opportunity, bedrooms were kept quiet and dark while sleep data were recorded via PSG.

The PSG data from the MSLT were visually scored according to the standard AASM PSG scoring criteria (2007). Sleep onset latency was scored as the time from the initiation of the MSLT trial ("lights out") to sleep onset. Sleep onset was identified by the first epoch of three 30second epochs that were unequivocally scored as sleep.

The MSLT yielded sleep onset latency scores for each MSLT trial. The four sleep onset latency scores for each participant were averaged into an average sleep onset latency score. Lower MSLT scores represent more sleepiness and higher MSLT scores represent less sleepiness. In concordance with the standardized MSLT protocol, each nap opportunity session was terminated at the end of the 20-minute session if no sleep occurred; however, if sleep did 
occur participants were allowed to sleep for 15 minutes, beginning at sleep onset, before the session was terminated (Afifi et al., 2005).

Subjective Rating of Sleep Onset Latency

Subjective ratings of sleep onset latency were assessed with a single question.

Participants rated their prediction of sleep onset latency for both themselves and their partner.

Prior to each MSLT trial, the respondents rated on a scale of 0 minutes -20 minutes according to half-minute intervals "How much time will it take you (your partner) to fall asleep?"

Surveys

Participants were verbally administered a 10-item sleep disorders screening survey (Appendix 2). Participants who indicated sleep disorder symptoms were provided information about community resources for evaluation and treatment (see Appendix 1). Questionnaires were self-administered by participants to examine demographic information and relationship history.

\section{Procedure}

Once potential participants were identified, a researcher set up a home visit when both parents in the dyad were available. During the home visit, a researcher described the research protocol in full detail, provided a tutorial on all of the equipment used in the protocol, described the surveys to the participants, and answered questions that participants had. Participants were administered informed consent and a Health Insurance Portability and Accountability Act (HIPAA) authorization. The procedures for the field-based and laboratory-based portions of the study are described below; a timeline for the research procedures is indicated on Figure 1. A list of constructs investigated in the current study and the instruments used to measure those constructs are indicated on Table 4. 


\section{Field-based protocol}

Participants began the protocol within their 3 to 8 week postpartum period. Participants wore an actigraph on their non-dominant wrist for 1 week continuously except when the actigraph might have become submerged in water. Participants recorded in the PDA sleep diary the times when they went to sleep and woke up from sleep. Participants also recorded when they took the actigraph off and put it back on. Participants completed the perception of sleep quality ratings each morning within 2 hours of awakening and before consuming caffeine — this is a standard procedure within the larger laboratory study in which many postpartum mothers concurrently participated.

For the perception of sleep quality ratings participants were counterbalanced into two groups; group A rated their own sleep quality first then their partner's, and group B rated their partner's sleep quality first then their own. The counterbalancing of groups was implemented to equally disperse error that may have resulted from order effects that may have resulted from rating one's self then their partner and vise versa. After participants completed the sleep quality ratings they answered the following request via voice note function on the Palm Pilot "Please describe why you woke up and what you did each time you woke up last night." The method of retrospectively describing one's nocturnal wake is susceptible to error due to forgetting or sleepiness. However, a retroactive account was a more appropriate alternative to recording one's nocturnal wake in real time because a real time recording would have potentially lead to more wakefulness and might have awakened a sleeping partner / infant. Actigraphy and PDA data were downloaded at the sleep research laboratory. 


\section{Laboratory based protocol}

Participants came to the sleep research laboratory approximately one hour after waking so that research personnel could outfit them with PSG sensors prior to their first MSLT nap opportunity. The first MSLT nap opportunity was conducted between two and three hours from the mother's and father's wake that morning. When one parent underwent the first MSLT nap opportunity first the other parent cared for their infant in a separate room from the laboratory. When the first parent completed their first MSLT nap opportunity, that parent took care of their infant while the other parent underwent their first MSLT nap opportunity. Subsequent nap opportunities followed that same order. The decision of which parent underwent their first MSLT nap opportunity first was based on participants' order of wake that morning; the parent who woke up first that morning took their first nap opportunity first.

Prior to each MSLT nap opportunity participants adjusted the ambient temperature and firmness of their bed to their preferred comfort. Next, participants provided a subjective rating of their and their partner's sleep onset latency. For subjective ratings of sleepiness participants were counterbalanced according to two groups; group A rated their own subjective sleepiness first then their partner's; conversely, group B rated their partner's subjective sleepiness first then their own. The reason for counterbalancing these measures was to equally disperse error that may have resulted from effects of order administration.

All PSG and MSLT procedures were conducted according to the standardized protocol (AASM, 2007; Littner et al., 2005) described above. During each MSLT nap opportunity the laboratory bedrooms had 30dB of white noise (Marpac SleepMate ${ }^{\circledR}$ 980A) to attenuate any potential ambient sounds. 
Following each MSLT nap opportunity, participants were disconnected from the Communication Unit and were allowed to engage in a variety of activities until the next MSLT nap opportunity. Activities included anything with low levels of vigor; such as, reading, using the internet, playing games, playing with their infant, tending to their infant, and watching television. Between the MSLT sessions participants were encouraged to remain out of bed and were not allowed to nap.

Between nap opportunities participants were verbally administered the sleep disorders screening survey by research personnel. Additionally, participants were provided with demographic and relationship history surveys.

Between two nap opportunities participants were provided with lunch from a local restaurant of their choice. After full participation in the study, each participant received an honorarium of $\$ 50$.

Analyses and Results

Overview of Results

An $\alpha$ level of .05 is considered statistically significant. The results are reported in the following main sections: 1) Data Management; 2) Preliminary Analyses; and 3) Main Analyses. Each main section is further described in subsections.

\section{Data Management}

A total of 42 participants participated in the study (see Participants: Study Sample section). All data were checked for missing data, outliers, and normality.

\section{Missing Data}

Actigraphy data was screened for equipment malfunction and missing data. Data were screened via visual inspection of actigram plots and corresponding numerical output. The 
inspection identified two instances of equipment malfunction /participant noncompliance with the protocol. One actigraph appeared to have malfunctioned during a mother's week of participation and data were not used for analyses. One father appeared to have not been compliant with the weekly objective sleep monitoring portion of the research protocol. The father appeared to have taken off the actigraph a few hours into the protocol and no objective sleep data were obtained throughout that week of monitoring.

MSLT data were screened for missing values. Data were screened via visual inspection of polysomnograms and research notes for unscoreable recordings and deviation from the MSLT protocol; the inspection identified one unscorable recording and two instances of deviation from protocol. One father's polysomnogram recording was unscorable because signals were recorded at 60 cycles per second; therefore, his sleep onset latency values were not used in the analyses. One sleep onset latency value from one mother's MSLT was excluded from analyses because the laboratory was not kept quiet during that nap opportunity. During the mother's nap opportunity, her partner entered the research laboratory with their crying baby. Upon the termination of the mother's nap opportunity, she reported that she couldn't fall asleep because she was worried about her infant's needs. One mother opted out of taking one nap opportunity during her MSLT because she had acid reflux after lunch. Missing and excluded data were removed from the corresponding mother's average daily sleep onset latency value.

Due to scheduling issues, one couple provided one day/night of the field-based protocol, participated in the laboratory-based protocol, then provided the remainder of the field-based protocol. Data from this couple were excluded from the analysis of research question two because of their change in order of protocol administration. 
Overall, $\mathrm{N}=19$ mothers and $\mathrm{N}=20$ fathers had averaged weekly actigraphy values; $\mathrm{N}=$ 21 mothers and $\mathrm{N}=21$ fathers had averaged weekly subjective sleep reports. Furthermore, $\mathrm{N}=$ 21 mothers and $\mathrm{N}=20$ fathers had average sleep onset latency values; $\mathrm{N}=21$ mothers and $\mathrm{N}=$ 21 fathers had averaged subjective sleep onset latency values. Missing were was handled via pairwise deletion when statistical analyses were calculated.

\section{Outliers}

A boxplot was used to identify outlying values for all variables that were three times above or below the interquartile range of the sampled variable. Any value identified as outlying was subsequently removed from calculation of the relevant analysis.

One mother had a nocturnal wake value, the week prior to her MSLT, that was approximately three times greater than the group (mothers') standard deviation plus the mean (group: $M=92.92, S D=33.74$; participant value $=193$ [Note: previous $M$ and $S D$ contain outlying value]). The value was removed from the analysis of sleep from the night prior to the MSLT and the previous week.

The difference between a mother's report on her partner's nocturnal wake time and her partner's report on his nocturnal wake time was approximately four times greater than the group (mothers') standard deviation plus the mean (group: $M=3.81, S D=84.66$; participant value: 339.57 [Note: previous $M$ and SD contain outlying value]). The father of this dyad likely misinterpreted the protocol instructions to report his nocturnal wake time, rather than this value representing the father's true perception of his nocturnal wake time. The value was removed from the analysis for examination of research question $5 \mathrm{H} \mathrm{b}$, as well as supplemental analyses. The father from the same dyad had a difference between his self-report of time awake and his actually time awake that was approximately four times greater than the group (fathers') standard 
deviation plus the mean (group: $M=1.98, S D=104.37$; participant value; 433.86 [Note:

previous $M$ and $S D$ contain outlying value]). Again, this father likely misinterpreted the protocol instructions to report his nocturnal wake time. The value was removed from examination of order effects on counterbalanced groups, the analysis for research question $5 \mathrm{H}$ a, as well as supplemental analyses.

\section{Normality}

The distribution of each variable was checked for skewness (symmetry of distribution) and kurtosis (peakedness of distribution). Skewness and kurtosis values were identified via inspection of histograms when plotted against normal distributions (Keppel \& Wickens, 2004). According to Keppel \& Wickens (2004), inspection of ahistogram against a normal distribution is an appropriate method evaluation of skewness and kurtosis. No distribution of study variables appeared different than a normal distribution. For skewness and kurtosis values for all variables are indicated on table 5.

\section{Preliminary Analyses}

Preliminary analyses were conducted to 1.) provide a descriptive overview of the data, 2.) determine whether infant feeding method and work status influenced MSLT sleep onset latency, 3.) examine the field-based protocol in descriptive detail, and 4.) examine the laboratory-based protocol in descriptive detail. The following sections address those issues, respectively.

\section{Descriptive Statistics}

Descriptive statistics were calculated for sleep and sleepiness variables for both mothers and fathers (Table 6). To provide an understanding of why participants were awake at night, mothers' and fathers' voice notes indicating why they awoke the previous night were transcribed and are shown in (Appendix 6). 
Approximately $35 \%$ of mothers napped at least once during the week-long field based data collection. Of those women who napped, naps occurred on $M=2.71, S D=1.50$ days and were $M=32.77, S D=24.39$ minutes in length. Approximately $35 \%$ of fathers napped at least once during the week-long field based data collection. Of those men who napped, naps occurred on $M=1.57, S D=.53$ days and were $M=22.86, S D=17.38$ minutes in length. Mothers and fathers who napped obtained sleep time from their naps that increased their average group nocturnal sleep time by approximately 12 and 9 minutes respectively.

\section{Infant Feeding Method and Work Status}

To examine whether infant feeding methods (exclusively breast, exclusively formula, or both), and / or work status (family leave, fulltime, part-time, unemployed) had an impact on sleep and sleepiness variables (24-hour sleep time, nocturnal sleep time, nocturnal wake time, fragmentation index, and MSLT sleep onset latency), a Factorial Multivariate Analysis of Variance (MANOVA) was conducted separately for mothers and fathers. For the MANOVAs, Infant feeding methods and work status were entered as the IVs and the sleep and sleepiness variables were entered as the DVs; Wilks' $\lambda$ criterion was used for interpretation. To further explore potential significant multivariate effects, univariate analysis of variance (ANOVA) and Tukey's posthoc analyses were calculated.

Among mothers, analyses could not be calculated because one work status group had fewer than two cases; that is, only one woman reported working 'full time' as a student. Therefore, to calculate these analyses the single 'full time' case was recoded as 'part time'. After recoding this case, the multivariate analysis was not significant for infant feeding method $F(10$, $16)=1.22, p=.35$, or work status $F(10,16)=1.59, p=.20$. 
Among fathers, neither feeding methods $F(10,16)=1.01, p=.47$, or work status $F(15$, $22.49)=1.64, p=.14$ had significant multivariate effects on the combined DVs. Additionally, there was no significant interaction among IVs on the combined DVs $F(5,8)=0.42, p=.83$.

\section{Field-Based Protocol}

Data from the field-based protocol were examined to identify 1.) the timing of participant self-administration of the morning questions, and 2.) if there were differences between the A and B groups on sleep quality ratings. The following sections address those issues respectively.

\section{Participant Compliance}

Data from the field-based protocol were examined to determine whether participants selfadministered the morning questions within two hours of wake as instructed. Mothers, selfadministered morning questions within $M=77, S D=53$ minutes from wake. Fathers selfadministered morning questions within $M=75, S D=53$ minutes from wake.

\section{Counterbalanced Groups}

Data from the field-based protocol were further examined to identify whether there were potential differences among the counterbalanced A and B groups for the self-administered sleep quality ratings. A separate One-Way MANOVA was conducted for both mothers and fathers. For the MANOVAs, group assignment (A or B) was entered as the IV; the sleep quality ratings (frequency of wake, cumulative length of nocturnal wake, and sleep quality) and the subjective sleepiness rating of self, and partner, were entered as the DVs; Wilks' $\lambda$ criterion was used for interpretation.

Among mothers, group assignment did not have a significant multivariate effect on the combined DVs $F(8,12)=1.40, p=.29$. Similarly, among fathers, group assignment did not have a significant multivariate effect on the combined DVs $F(8,11)=1.68, p=.21$. 


\section{Laboratory Based Protocol}

Data from the laboratory-based protocol were examined to identify 1 ) the timing of the first nap opportunity, 2) the validity of the PSG scoring for the MSLT sleep onset latency, 3) whether the previous night sleep had an effect on the MSLT results the following day, and 4) whether sleep disorder symptoms were associated with MSLT results. The following sections address those issues respectively.

\section{Timing of First Nap Opportunity}

Data from the laboratory-based protocol were examined to determine whether the first nap opportunity occurred within 3 hours from wake that morning. Mothers' first nap opportunity occurred within $M=211, S D=45$ minutes (3 hours 31 minutes) from wake. Fathers' first nap opportunity occurred within $M=197, S D=45$ minutes (3 hours 17 minutes) from wake.

Potential associations were examined among latency from wake to the first MSLT nap opportunity and the MSLT identified sleep onset latency value. Bivariate correlations were calculated among these values separately for both mothers and fathers. Among mothers, the amount of time elapsed from wake to the first MSLT nap opportunity was significantly associated with MSLT identified sleep onset latency $r=.45, p<.05$. As latency from wake to the first nap opportunity increased, sleep onset latency increased. Among fathers, the amount of time elapsed from wake to the first MSLT nap opportunity was not significantly associated with MSLT identified sleep onset latency $r=-.03, p=.91$. The latency from wake to the first MSLT nap opportunity did not significantly differ among mothers and fathers $F(1,18)=2.48, p=13$.

\section{Reliability of PSG Scoring}

Data from the laboratory-based protocol were further examined to determine the interreliability of the PSG scores for the MSLT sleep onset latency. Two independent analysts blind 
to each other's ratings, and one was blind to participant-dyad, scored sleep onset latency according to AASM standard criteria (AASM, 2005). Initial agreement was $>90 \%$ ([number of ratings identified as exact agreement / total number of ratings] X 100), and overall, scorers differed by $M=69, S D=146$ seconds. All discrepancies between analysts were settled and the agreement on the initiation of sleep onset latency reached $100 \%$.

\section{Previous Night Sleep and MSLT Results}

Data were examined to determine whether sleep from the night prior to the MSLT differed from sleep from the previous week, and whether this difference was associated with MSLT sleep onset latency. Specifically, sleep values from night 7 of the field-based study (night before the MSLT) were compared to the sleep value averages from nights 1 through 6 (baseline sleep) with the use of repeated measures ANOVAs. Next, the residuals among the night prior to the MSLT and the baseline night, for each sleep value, were examined for associations with MSLT sleep onset latency values. All statistics for these analyses are shown on Table 7.

Among mothers, 24-hour sleep time and nocturnal sleep time differed between the baseline sleep and the sleep from the night prior to the MSLT. However, the residuals among sleep values were not significantly associated with MSLT sleep onset latency values. The removal of one mothers' outlying nocturnal wake value impacted the comparison between baseline sleep and sleep from the night prior to the MSLT. With the extreme outlying value there was a significant difference, with out the value there was not a significant difference. Among fathers, no sleep values differed among the baseline sleep and the night prior to the MSLT. Furthermore, the residuals among sleep values were not associated with MSLT sleep onset latency values. 
Among parents, sleep on the night prior to the MSLT was shorter in length compared to sleep from the previous week; however, no other sleep indices were different. Parents may have shortened their sleep by going to bed later or waking up early on the night prior to the MSLT because: 1) they prepared infant care supplies, 2) wanted to start the study early, 3) they couldn't sleep because they were anxious about the study, 3) because they knew they were able to nap on the following day so they stayed up later than normal. However, since the residuals among the baseline and the night prior to MSLT were not associated with the MSLT values, sleep from the night prior to the MSLT was not presumed to impact the MSLT results. Lack of statistical power is a possible explanation for not identifying significant results. However, among associations no $p$ value approached significance and all correlations were small; therefore, albeit no statistical significance, the clinical significance of these small associations is negligible.

\section{Sleep Disorder Symptoms and MSLT Results}

Data were examined to determine whether sleep disorder symptoms were associated with MSLT identified sleep onset latency. Bivariate correlations were calculated among each sleep disorder symptom value and MSLT identified sleep onset latency, separately, for mothers and fathers. No sleep disorder symptom value was associated with MSLT sleep onset latencies for mothers or fathers (Table 8).

\section{Main Analyses}

The main analyses for each research aim are reported in sections that correspond to the previously described research questions. The main analyses for each research question are described along with the hypothesized results. The examination of each research question is followed by supplemental analyses for that research question. 


\section{Research Aim 1}

\section{Research Question 1: Main analyses}

The purpose of research question one was to identify how sleep indices differ among mothers and fathers during the first postpartum month. To address this research question two one-way repeated measures ANOVAs were calculated. For both ANOVAs parental status mother or father — was the IV; for the first ANOVA 24-hour sleep time was entered as the DV, and for the second ANOVA nocturnal sleep fragmentation was entered as the DV.

The hypothesis ( $\mathrm{H} 1 \mathrm{a})$ that mothers had less 24-hour sleep time than fathers was examined. Mothers $(M=424.83, S D=42)$ had significantly $F(1,18)=17.31, p<.01, d=1.30$ more 24-hour sleep time than fathers $(M=375.14, S D=34.06)$. Thus, H1 a was rejected (see Table 6).

The hypothesis ( $\mathrm{H} 1 \mathrm{~b})$ that mothers had more nocturnal sleep fragmentation than fathers was examined. Mothers $(M=18.86, S D=3.34)$ had significantly more $F(1,18)=13.17, p<.01$, $d=1.12$ sleep fragmentation than fathers $(M=14.34, \mathrm{~S} D=4.62)$. Thus, $\mathrm{H} 1 \mathrm{~b}$ was retained (see Table 6).

Research question 1: Supplemental analyses. Analyses were conducted to identify how additional sleep indices differed among mothers and fathers. The same series of repeated measures ANOVAs as described in the previous section were calculated; however, additional DVs included nocturnal sleep time, nocturnal wake time, and sleep efficiency. Overall, mothers had significantly more nocturnal sleep time and nocturnal wake time than fathers (see Table 6).

\section{Research Question 2: Main Analyses}

The purpose of research question two was to identify whether objectively measured sleep indices for one week predicted mothers' and fathers' subsequent objectively measured 
sleepiness. To address research question two, separate stepwise regressions were calculated for mothers and fathers; objectively measured sleepiness was the DV, and 24-hour total sleep time, nocturnal sleep time, nocturnal wake time, sleep fragmentation, and sleep efficiency were the IVs. A stepwise regression was used because the research question is exploratory; therefore, the order of predictive variables was statistically determined.

The hypothesis (H2) that objectively measured sleep indices predict sleepiness among postpartum mothers and fathers was examined. Among mothers, no objective sleep indices significantly predicted sleepiness. Among fathers, no objective sleep indices significantly predicted sleepiness. Thus, H2 was rejected.

Research question 2: Supplemental analyses. Analyses were conducted to identify whether subjectively measured sleep indices for one week predicted mothers' and fathers' subsequent objectively measured sleepiness. Stepwise regressions were calculated for mothers and fathers; objectively measured sleepiness was the DV; frequency of wake, cumulative length of nocturnal wake, and sleep quality were the IVs. Among mothers, no subjectively reported sleep indices significantly predicted objectively measured sleepiness. Similarly, among fathers no subjectively reported sleep indices significantly predicted objectively measured sleepiness. Research Question 3: Main Analysis

The purpose of research question three was to identify whether postpartum mothers or fathers had higher levels of objectively measured sleepiness. To address this research question a one-way repeated measures ANOVA was calculated. Parental status — mother or father — was the IV; MSLT identified sleep onset latency was the DV.

The hypothesis $(\mathrm{H} 3)$ that mothers will have higher levels of sleepiness than fathers was examined. Fathers $(M=11.80, S D=4.60)$ had significantly $F(1,19)=11.85, p<.005, d=0.88$ 
higher levels of sleepiness than mothers $(M=8.03, S D=3.92)$. Thus, $\mathrm{H} 3$ was rejected (see Table 6). In fact, the opposite hypothesized direction was found.

Research Question 3: Supplemental analyses. Descriptive analyses were calculated to examine the percentage of mothers and fathers who demonstrate extreme pathological sleepiness, extreme sleepiness that may or may not be pathological, and normative sleepiness (Afifi et al., 2005). Among mothers, $10 \%$ were within the extreme pathological sleepiness range, $29 \%$ were in the extreme sleepiness that may or may not be pathological range, and $62 \%$ were in the normative range (see Figure 2 a). Among fathers, 25\% were within the extreme pathological sleepiness range, $50 \%$ were in the extreme sleepiness that may or may not be pathological range, and $25 \%$ were in the normative range (see Figure $2 \mathrm{~b}$ ).

\section{Research Aim 2}

\section{Research Question 4: Main Analyses}

The purpose of research question four was to identify whether mothers or fathers were more accurate in reporting their and their partner's levels of sleepiness. To address this research question a one-way repeated measures ANOVA was calculated. Parental status - mother or father - was the IV; the DV was the difference scores between subjective ratings of sleepiness and objective sleepiness scores from the MSLT, these measures were on the same scale.

The hypothesis (H4 a) that postpartum fathers' self-reports of sleepiness will be more accurate than postpartum mothers' self-reports was examined. Fathers difference scores $(M=$ 3.09, $S D=5.07$ ), between self-reports of sleepiness and objective measures of sleepiness, were not significantly different $F(1,19)=3.78, p=.07, d=0.57$ from mothers differences scores $(M$ $=.55, S D=3.78)$. Thus, $\mathrm{H} 4$ a was rejected (see Table 9). In fact, a trend was apparent in the opposite direction. 
The hypothesis ( $\mathrm{H} 4 \mathrm{~b})$ that postpartum fathers will be more accurate than postpartum mothers in reporting their partner's sleepiness was examined. Fathers' difference scores $(M=$ $.49, S D=4.62$ ) between subjective reports on partner's sleepiness and objective measures of their partners' sleepiness, were significantly different $F(1,19)=9.82, p<.01, d=0.87$ and more accurate than mothers' difference scores $(M=3.68, S D=5.01)$. Thus, H4 b was retained (see Table 9).

Research question 4: Supplemental analyses. Analyses were conducted separately for mothers and fathers to examine the accuracy of their self-reports of sleepiness with their objectively measured sleepiness. Bland-Altman plots (Altman \& Bland, 1983) with references to objectively measured sleepiness were created to illustrate mother (Figure 3 a) and father (Figure $3 \mathrm{~b})$ differences respectively; accuracy of mother and father self-reports of sleepiness are shown on Figure 3 c. Overall, mothers underestimated their sleepiness $(M=.28, S D=3.90$ minutes); $38 \%$ of mothers were within $\leq 2.50$ minutes, and $86 \%$ were $\leq 5$ minutes of accuracy when reporting their sleep onset latency. Likewise, fathers underestimated their sleepiness $(M=3.09$, $S D=5.07$ minutes); $30 \%$ of fathers were within $\leq 2.50$ minutes, and $50 \%$ were $\leq 5$ minutes of accuracy when reporting their sleep onset latency.

Analyses were conducted separately for mothers and fathers to examine the accuracy of their reports on their partner's sleepiness with their partner's objectively measured sleepiness. Bland-Altman plots with references to objectively measured sleepiness were created to illustrate mother (Figure 4 a) and father (Figure 4 b) differences respectively; accuracy of mother and father reports of their partner's sleepiness are shown on Figure 4 c. Overall, mothers underestimated their partner's sleepiness $(M=3.68, S D=5.01$ minutes $) ; 30 \%$ of mothers were within $\leq 2.50$ minutes, and $50 \%$ were $\leq 5$ minutes of accuracy when reporting their partner's 
sleep onset latency. Conversely, fathers overestimated their partner's sleepiness $(M=-.80, S D=$ 4.72 minutes); $28 \%$ of fathers were within $\leq 2.50$ minutes, and $81 \%$ were $\leq 5$ minutes of accuracy when reporting their partner's sleep onset latency.

\section{Research Question 5: Main Analyses}

The purpose of research question five was to identify whether mothers or fathers were more accurate in reporting the amount of time they and their partner were awake the previous night. To address this research question a one-way repeated measures ANOVA was calculated. Parental status - mother or father — was the IV; the DV was the difference scores between subjective ratings of time awake the previous night and objective values from actigraphy.

The hypothesis (H5 a) that fathers' self-reports of the amount of time they were awake during the previous night will be more accurate than postpartum mothers' self reports was examined. Fathers difference scores $(M=-10.50, S D=33.96)$, between self-reports of amount of time they were awake during the previous night and objective measures of wake time, were significantly different $F(1,17)=9.66, p<.01, d=0.86$ and more accurate than mothers differences scores $(M=28.95, S D=55.59)$. Thus, H5 a was retained (see Table 9).

The hypothesis (H5 b) that fathers' will be more accurate than postpartum mothers' in reporting the amount of time their partner was awake the previous night was examined. Fathers difference scores $(M=1.41, S D=40.06)$, between subjective reports on partner's time awake and objective measures of their partners' time awake, were not significantly different $F(1,17)=$ $2.82, p=.11, d=0.46$ than mothers difference scores $(M=-15.17, S D=31.57)$. Thus, H5 b was rejected (see Table 9).

Research question 5: Supplemental analyses. Analyses were conducted separately for mothers and fathers to examine the accuracy of their self-reports of nocturnal wake time with 
their objectively measured nocturnal wake time. Bland-Altman plots (Altman \& Bland, 1983) with references to objectively measured nocturnal wake were created to illustrate mother (Figure 5 a) and father (Figure 5 b) differences respectively; accuracy of mother and father self-reports of nocturnal wake time are shown on Figure $5 \mathrm{c}$. Overall, mothers overestimated their nocturnal wake time $(M=30.32, S D=56.96$ minutes $) ; 35 \%$ of mothers were within $\leq 25$ minutes, and $70 \%$ were $\leq 50$ minutes of accuracy when reporting their nocturnal wake time. Conversely, fathers underestimated their nocturnal wake time $(M=-10.23, S D=33.02$ minutes $) ; 47 \%$ of fathers were within $\leq 25$ minutes, and $84 \%$ were $\leq 50$ minutes of accuracy when reporting their nocturnal wake time.

Analyses were conducted separately for mothers and fathers to examine the accuracy of their reports on their partner's nocturnal wake time with their partner's objectively measured nocturnal wake time. Bland-Altman plots with references to objectively measured nocturnal wake were created to illustrate mother (Figure 6 a) and father (Figure 6 b) differences respectively; accuracy of mother and father reports of partner's nocturnal wake time are shown on Figure 6 c. Overall, mothers underestimated their partner's nocturnal wake time $(M=-13.87$, $S D=31.20$ minutes); $42 \%$ of mothers were within $\leq 20$ minutes, and $74 \%$ were $\leq 40$ minutes of accuracy when reporting their partner's nocturnal wake time. Conversely, fathers overestimated their partner's nocturnal wake time $(M=3.64, S D=42.23$ minutes $) ; 40 \%$ of fathers were within $\leq 20$ minutes, and 55\% were $\leq 40$ minutes of accuracy when reporting their partner's nocturnal wake time. 


\section{Discussion \\ Overview of Main Findings}

\section{Aim 1: Objective Sleep and Sleepiness}

Mothers obtained significantly more 24-hour sleep time than fathers; however, mothers also had significantly more nocturnal sleep fragmentation than fathers. Mothers' and fathers' objectively measured sleep did not predict their objectively measured sleepiness. Fathers had significantly higher levels of objectively measured sleepiness than mothers.

\section{Aim 2: Perception of self and partner}

Mothers' and fathers' accuracy in reporting their own levels of sleepiness did not differ from each other; mothers' reports were closer to objective measures than fathers' reports. Mothers' and fathers' accuracy in reporting their partner's levels of sleepiness significantly differed; fathers' reports were closer to objective measures than mothers' reports. Mothers' and fathers' accuracy in reporting their own nocturnal wake time significantly differed; fathers' reports were closer to objective measures than mothers' reports. Mothers' and fathers' accuracy in reporting their partner's nocturnal wake time did not differ from each other; fathers' reports were closer to objective measures than mothers' reports.

\section{Review and Interpretation of Study Findings: Aim 1}

\section{Research Question 1: Difference in Sleep Indices Among Mothers and Fathers}

Hypothesis $1 \mathrm{a}$. The hypothesis that mothers will have less 24-hour sleep time than fathers was rejected. Mothers actually had significantly more 24-hour sleep time than fathers.

The rationale for the direction of this hypothesis was that pilot data analyses - as part of the original research proposal (data were used from a larger laboratory study) - were calculated and showed that our laboratory's sample of first time postpartum mothers did not nap often 
during the early postpartum period. The observation from dissertation pilot data analyses is supported by previous research, which indicates that mothers with young infants do not frequently nap (Cottrell \& Karraker, 2002). Furthermore, at the time of the original research proposal, ongoing work in our laboratory indicated that postpartum mothers demonstrated high levels of sleep fragmentation during their early postpartum period. Therefore, among the current study's projected sample of postpartum women, high levels of sleep fragmentation were expected to occur, and mothers were not expected to nap often.

Furthermore, Gay and colleagues found that mothers had less nocturnal sleep time, but more 24-hour sleep time than fathers (Gay et al., 2004); that is, mothers took daytime naps to inflate their 24-hour sleep time to levels higher than fathers. Additionally, mothers in their study had significantly more nocturnal sleep fragmentation than fathers. On the basis of these findings, along with pilot work that indicated that mothers rarely napped, mothers were expected to have less 24-hour sleep time than fathers; yet this was not the case in regard to the current findings. Rather, the current findings indicate that mothers obtained significantly more 24-hour sleep time than fathers. This result is congruent with previous evidence that postpartum mothers obtained more 24-hour sleep time than fathers (Gay et al., 2004); however, the results from the current study are similar to results from the work of Gay and colleagues due to markedly different reasons. As described in the supplemental analyses (Table 6), mothers also obtained more nocturnal sleep time than fathers, which is incongruent with previous reports that fathers obtained more nocturnal sleep time than mothers (Gay et al., 2004). Furthermore, both mothers and fathers had similar nap profiles; the nap profiles did not appear to influence the differences in 24-hour and nocturnal sleep time among mothers and fathers as seen in previous work (Gay et al., 2004). 
The current data indicate that when compared to fathers, mothers either had an earlier sleep onset time, later wake time, or both. Preliminary analyses indicated that neither work status, nor infant feeding method appeared to have a significant effect on 24-hour, or nocturnal sleep time among mothers or fathers. The potential effects of work status and infant feeding method were not detected; most likely because of the small sample sizes within each group. Conclusions about the effect of work status and feeding methods on sleep and sleepiness should not be made from the current work because the research design was not adequate to address those issues. These variables are in fact important to explore the mechanisms for why mothers and fathers sleep differently and express different sleepiness levels. Analyses will be conducted to further explore potential profiles of sleep and wake timing that may identify when, and how, mothers obtain more sleep time than fathers.

Hypothesis $1 \mathrm{~b}$. The hypothesis that mothers will have more nocturnal sleep fragmentation than fathers was supported. Mothers in fact had more nocturnal sleep fragmentation than fathers.

The rationale for the direction of this hypothesis was that postpartum mothers were expected to be more affected by hormonal alterations following delivery (Manber \& Armitage, 1999), and mothers usually provide a greater degree of nocturnal caregiving to their infant than fathers (Kennedy, Gardiner, Gay, \& Lee, 2007). Additionally, the expected results were consistent with findings from previous research that examined postpartum parents at approximately three weeks postpartum (Gay et al., 2004).

Adverse effects of sleep fragmentation include, but are not limited to, impaired health (Spiegel, Knutson, Leproult, Tasali, \& Van Cauter, 2005), mood, and cognitive performance 
(Martin, Engleman, Deary, \& Douglas, 1996). Therefore, mothers may be more at risk for adverse effects of sleep fragmentation than fathers.

The results regarding nocturnal sleep fragmentation were confirmed by supplemental analyses that indicated that mothers also spent more time awake at night compared to fathers (Table 6). Importantly, nocturnal wake time and nocturnal sleep fragmentation are different measures of the same construct—wake during a sleep period. Predictors of nocturnal wake and purposes of nocturnal wake are important to identify to better understand sleep fragmentation among postpartum parents, and to potentially guide postpartum sleep interventions to ultimately decrease sleep fragmentation among new parents.

The reason for nocturnal awakenings among postpartum parents was not a primary focus of the current study. Therefore, mechanisms for nocturnal wake cannot be conclusively identified, although certain causes can be inferred.

Inspection of the subjective reports of nocturnal wake (Appendix 6) indicated that mothers' entries typically pertained to infant caregiving tasks; whereas fathers' entries pertained to being awakened by infant signaling. Therefore, mothers may have engaged in infant caregiving tasks at the expense of having more wake during their sleep periods than fathers; comparatively, fathers may not have engaged in as many caregiving tasks as mothers and had less wake during their sleep periods than mothers. If the previous observation is correct, one may speculate a functional component of the proposed nocturnal behaviors; fathers may obtain consolidated sleep through the night so that they become rested for a structured work schedule the following day, while mothers engage in nocturnal caregiving responsibilities because they may not have an as-structured work schedule the following day due to maternity leave. As 
previously mentioned, firm conclusions cannot be drawn regarding the influence of work status on sleep profiles among mothers and fathers.

\section{Research Question 1: Summary}

Mothers obtained more 24-hour sleep, and more nocturnal sleep, but also had more nocturnal sleep fragmentation than postpartum fathers. Results from the current research question provide descriptive sleep indices of postpartum parents during their early postpartum period, approximately when mothers would be expected to return to work and fathers would be back to work. These descriptive reports, within the context of postpartum parents' objective levels of sleepiness, indicate that postpartum parents' sleep could be improved to curtail

sleepiness and potential sleepiness associated performance impairments. Analyses are planned to examine the associations among sleep profiles with sleepiness, fatigue, and performance variables.

\section{Research Question 2: Sleep and Prediction of Sleepiness}

Hypothesis 2 . The hypothesis that objectively measured sleep indices would predict sleepiness among postpartum mothers and fathers was rejected. Neither total sleep time nor sleep fragmentation predicted subsequent sleepiness among mothers or fathers.

The rationale for the direction of this hypothesis was that increases in sleep disturbance are known to increase daytime sleepiness as measured by the MSLT (Afifi et al., 2005). Furthermore, no research has clearly indicated what sleep parameters may have a greater effect on sleepiness among postpartum parents; therefore, the hypothesis was exploratory.

The current findings indicated that 24-hour total sleep time, nocturnal sleep time, nocturnal wake time, sleep fragmentation, and sleep efficiency did not predicted subsequent sleepiness among mothers or fathers. Likewise, via supplemental analyses, subjective sleep 
indices that included frequency of nocturnal wake, amount of nocturnal wake, and sleep quality did not predict subsequent sleepiness among mothers or fathers.

Higher levels of sleep deprivation have been repeatedly associated with objective sleepiness measured via the MSLT. Among normative samples of adults, increased grades of experimentally induced partial sleep deprivation (Rosenthal, Roehrs, Rosen, \& Roth, 1993) and sleep fragmentation (Levine, Roehrs, Stepanski, Zorick, \& Roth, 1987) had an effect on subsequent MSLT-identified sleepiness.

The results from the current study might be explained by the parents' prolonged duration of sleep disturbance, and the cumulative effect that sleep disturbance may have on postpartum sleepiness levels. If so, average weekly sleep values may not capture such a dynamic relation. Thus, a multilevel modeling analysis that would control for the dependency of each night's sleep profile with the previous night's sleep profile, for a prolonged portion of the postpartum period, may be a more robust analysis to capture predictors of sleepiness among postpartum parents.

\section{Research Question 2: Summary}

Neither objective nor subjective sleep indices significantly predicted objective sleepiness values among postpartum parents. Future research designs should be developed to identify how the cumulative effect of prolonged sleep disturbance may have an effect on postpartum sleepiness.

\section{Research Question 3: Sleepiness Levels}

Hypothesis 3. The hypothesis that mothers would have higher levels of sleepiness than fathers was rejected. Fathers actually had significantly more sleepiness than mothers.

The rationale for the direction of this hypothesis was that postpartum mothers were expected to have less total sleep and more sleep fragmentation than fathers, and thus, higher 
levels of sleepiness. Furthermore, according to Bonnet's Sleep Continuity Theory, a consolidated interval of sleep is necessary for sleep to be restorative (Bonnet, 1986). Therefore, according to Bonnet's theory, if mothers' sleep was more fragmented then fathers' sleep, and mothers obtained the same or less sleep time than fathers, mothers would demonstrate higher levels of sleepiness.

Mothers had a sleep onset latency of approximately twelve minutes and fathers approximately eight minutes, each with fairly large standard deviations. The sleep onset latency of mothers is on the border of extreme sleepiness that may or may not be pathological, whereas fathers' values are within that range of extreme sleepiness that may or may not be pathological. The current results can be interpreted in one of two ways. First, mothers may be more resistant to falling asleep than fathers, especially in the laboratory environment. Second, objective propensities to fall asleep were in fact functions of each parent's sleep profile.

Mothers may have been more resistant against falling asleep than fathers because mothers may have adapted to their levels of sleepiness. As reviewed by Roehrs and colleagues, there is evidence that adaptation to chronic sleepiness can occur (Roehrs, Carskadon, Dement, \& Roth, 2005). Specifically, under variable time schedules of a fixed amount of sleep loss (i.e., four nights, two nights, and one night), participants demonstrated a resiliency on alertness, memory, and performance tasks when sleep loss accumulated over a longer rate of time, suggesting adaptation to sleep loss (Drake, Roehrs, Burduvali, Bonahoom, Rosekind, \& Roth, 2001). Therefore, mothers may have adapted to their high levels of sleep fragmentation to a point where they express resilience to their high levels of sleep fragmentation as indicated by their longer sleep onset latencies than fathers. Additionally, research on insomnia has identified a connection between anxiety and sleep onset latency. Specifically, persons with generalized anxiety 
demonstrate longer sleep-onset insomnia (Viens, De Koninck, Mercier, St-Onge, \& Lorrain, 2003), and postpartum women appear to have higher levels of generalized anxiety than the general population of women (Ross, \& McLean, 2006). Postpartum mothers may have experienced heightened levels of anxiety when they attempted to nap in the novel environment of the sleep research laboratory. Therefore, postpartum mothers who had sleep disturbance were potentially susceptible to anxiety while in the novel laboratory environment where the MSLT was conducted, and thus, mothers may have demonstrated extended sleep onset latencies as an expression of their heightened levels of anxiety. Along this line, fathers may not have experiences heightened anxiety and instead interpreted MSLT nap opportunities as true opportunities to sleep because they were 'off-duty.'

Mothers' and fathers' objective propensity to fall asleep may have reflected their sleep profiles. Fathers may have had a higher objective propensity to fall asleep than mothers because fathers had less 24-hour sleep time and less nocturnal sleep time than mothers. As expected, the group (i.e., mother or father) with lower sleep time expressed greater sleepiness. However, sleep fragmentation was also expected to have an effect on sleepiness levels. Although mothers had more sleep fragmentation than fathers, and fathers had a greater propensity to fall asleep than mothers, the two observations are not incompatible. Mothers may have obtained enough sleep time, to partially counter the negative effects that sleep fragmentation had on their sleep; that is, over and beyond the adverse effects that a shorter sleep time had on fathers' sleep.

\section{Research Question 3: Summary}

Both mothers and fathers demonstrated high levels of objectively measured sleepiness, but fathers demonstrated higher objective levels of sleepiness than mothers. By indexing the levels of sleepiness among postpartum parents, the effects of sleep disturbance on this 
demographic are better understood. Most importantly, the sleepiness values reported within the current study reveal a societal problem at large. Following the birth of a child, postpartum parents almost immediately have to go back to work to provide for their family; yet, parents have high levels of sleepiness and empirical evidence clearly identifies the adverse effects of sleepiness on safety and performance in the workforce (Walsh et al., 2005). Consequently, the current FMLA policy (United States Department of Labor, 1993) is not practical for most families without putting at least one postpartum parent back into the workforce, albeit with likely sleepiness associated impairments. Experts describe an urgent need for changes in policy that will increase practices that facilitate alertness that will in turn stimulate safety, health, and productivity within society (Rosekind, 2005); however, postpartum parents were not discussed among the populations that are susceptible to sleepiness. Therefore, policy makers along with social and behavioral scientists may need to consider the current FMLA policy for new families in light of these findings.

The amount of sleep that mothers obtained to counter the effects of their sleep fragmentation, as demonstrated by their MSLT scores, demonstrates the restorative property of sleep. Future studies could examine the dose-response in the amount of sleep needed to make up for the effects of different levels of sleep fragmentation, especially among sleep disturbed populations.

\section{Review and Interpretation of Study Findings: Aim 2}

\section{Research Question 4: Accuracy in Report of Sleepiness}

Hypothesis 4 a. The hypothesis that postpartum fathers' self-reports of sleepiness will be more accurate than postpartum mothers' self-reports was rejected. The difference between father's self-report of sleepiness and fathers' objective sleepiness was not significantly different, 
or more accurate, than the difference between mother's self-report of sleepiness and mothers' objective sleepiness.

The rationale for the direction of the hypothesis was that disturbed sleep is known to have an adverse effect on judgment. Specifically, as indicated above, as sleep disturbance accumulates, which may result in subsequent daytime sleepiness, individuals become less accurate judges of their own levels of sleepiness (Vaughn \& D'Cruz, 2005). Postpartum fathers' self-reports of their own sleepiness were expected to be more accurate than mothers' self-reports of their own sleepiness because fathers were expected to have less sleep disturbance and be less sleepy than mothers.

The rationale for establishing the direction for the hypothesis was acceptable in light of the findings because the expected directions of sleep and sleepiness values among mothers and fathers were reversed and so were the accuracy reports. Although not significant, mother's selfreports of sleepiness were closer to their own objective sleepiness values than father's selfreports of their own objective sleepiness values, potentially because mothers had more sleep time and less sleepiness than fathers. Also, both mothers and fathers underestimated their levels of sleepiness. Future research could identify ways to improve parental perceptions of their own state of sleepiness to provide new parents with an ability to accurately identify their sleep associated impairments, especially prior to their engagement in a risk associated task. Additionally, these findings may stimulate researchers to investigate parental perceptions of which member within the parental dyad is less impaired due to sleepiness; or ultimately, which parent is better suited to engage in a certain task (e.g., infant caregiving, driving) during a given time. 
Hypothesis $4 \mathrm{~b}$. The hypothesis that postpartum fathers will be more accurate than postpartum mothers in reporting their partner's sleepiness was supported. The difference between fathers' report of mothers' sleepiness and mothers' objective sleepiness was significantly different, and more accurate, than the difference between mothers' report on fathers' sleepiness and fathers' objective sleepiness.

The rationale for the direction of this hypothesis was that, as previously mentioned, one's judgment is compromised as sleep disturbance increases (Vaughn \& D’Cruz, 2005). Furthermore, fathers were expected to have less sleep disturbance and less sleepiness than mothers and therefore, fathers were expected to have more accurate reports on partner's sleepiness than mothers.

Fathers did not have less sleep disturbance and less sleepiness than mothers; yet, father's reports on mother's sleepiness were more accurate than mother's reports on father's sleepiness. Mothers reported that fathers were less sleepy than they objectively were, and conversely, fathers reported that mothers were sleepier than they objectively were. The previous rationale for the direction of the current hypothesis is not suitable. Alternatively, mothers may not have had an accurate representation of how sleepy fathers were, because mothers may have perceived fathers as obtaining more restorative sleep than themselves; thus, mothers' perception that fathers had less sleepiness than themselves. Mothers may not have known how sleepy fathers were because fathers may not have been expressive about their sleepiness state. Conversely, fathers may have had a clear understanding about how sleepy mothers were because mothers may have been expressive about their sleepiness state. Additionally, fathers may have known when mothers got up a night; whereas, mothers may not have known if fathers woke up when mothers woke up to care for the baby. Future research could investigate ways to improve dyadic perceptions of 
partner sleepiness to ultimately identify how familial dyads can work together to optimize their performance on caregiving and household tasks; thus, decreasing the adverse effects of sleepiness on everyday life among new families.

\section{Research Question 4: Summary}

The current findings demonstrate that the accuracy of mothers' and fathers' self-reports of sleepiness were not significantly different, but mothers' reports were closer to objective measures than fathers' reports. Furthermore, the accuracy of mothers' and fathers' reports on their partner's sleepiness were significantly different; fathers' reports were closer to mother's objective measures than mother's reports were to fathers' objective measures. Although the clinical significance of these findings can not be determined from the current reports, these reports provide an initial description of parental perception of sleepiness. These findings may lay the framework to better understand dynamic family interactions among new parents - a necessary step towards looking at new families as an integrated whole. Ultimately, productivity and safety within a postpartum household could be maximized by understanding parental perceptions of sleepiness. For example, if one parent is better at evaluating their levels of sleepiness, or their partner's levels of sleepiness, then that parent may be best suited to identify which parent in the dyad is more fit to carry out a specific caregiving task, or household responsibility, while the other parent point their efforts in a different direction.

Research Question 5: Accuracy in Report of Nocturnal Wake

Hypothesis 5 . The hypothesis that fathers' self-reports of the amount of time they were awake during the previous night would be more accurate than postpartum mothers' self reports was supported. The difference between fathers' self-report of nocturnal wake and fathers' 
objective nocturnal wake was significantly different from and more accurate than the difference between mothers' self-report of nocturnal wake and mothers' objective nocturnal wake.

The rationale for the direction of this hypothesis was that, as previously described, sleep disturbance has a negative effect on judgment. Furthermore, sleep deprivation impairs abilities to form new memories (Yoo, Hu, Guijar, Jolesz, \& Walker, 2007). Therefore, postpartum fathers' self-reports of previous night sleep were expected to be more accurate than mothers' reports because fathers were expected to have less sleep disturbance and less sleepiness than mothers.

The rationale for the direction of this hypothesis is not suitable; fathers did not have less sleep disturbance and less sleepiness than mothers, but they did have significantly more accurate reports of nocturnal wake time than mothers. However, several plausible interpretations may apply to these findings. When compared to fathers, mothers had more objectively measured nocturnal wake time and greater levels of sleep fragmentation; therefore, greater levels of nocturnal wake time and sleep fragmentation may have lead to poorer memory recall. Additionally, when compared to objective measures, mothers overestimated their own nocturnal wake times and fathers underestimated their own nocturnal wake times. Each parent may have overestimated the direction of their own nocturnal wake time as referenced to their perception of their partner's nocturnal wake time. Future research should be conducted to better understand parental perceptions of their own sleep. An accurate representation of how one thinks they slept is important because subjective sleep quality predicts subjective daytime sleepiness (Insana \& Montgomery-Downs, 2009). Therefore, if mothers routinely overestimate their own nocturnal wake time, mothers may be more susceptible to the adverse consequences of sleepiness (e.g., mood disturbance or fatigue) than if they were more accurate at reporting their nocturnal wake time. If fathers routinely underestimate their own nocturnal wake time, fathers may perceive 
themselves as less sleepy than they are, and therefore engage in a risk associated task while objectively impaired, thus putting themselves (or their family) at undue risk.

Hypothesis $5 \mathrm{~b}$. The hypothesis that fathers will be more accurate than postpartum mothers in reporting the amount of time their partner was awake the previous night was rejected. The difference between the father's report of the mother's nocturnal wake time and the mother's objective nocturnal wake time was not significantly different than the difference between the mother's report of the father's nocturnal wake time and the father's objective nocturnal wake time.

The rationale for the direction of this hypothesis was similar to the previous rationale. Fathers were expected to have fewer memory and perception impairments due to less sleep disturbance and less sleepiness than mothers, and therefore fathers were expected to have more accurate reports on partner's nocturnal wake time than mothers.

The differences in accuracy of reporting partners' nocturnal wake times did not significantly differ among mothers and fathers; however, fathers' difference scores were smaller than mothers' difference scores. Therefore, fathers' reports of their partners' nocturnal wake times may be more accurate than mother's reports of their partners' nocturnal wake time. When compared to objective measures, mothers underestimated fathers' nocturnal wake times and fathers overestimated mothers' nocturnal wake time. Once again, each parent may have overestimated the direction of their partners' nocturnal wake time as referenced to their perception of their own nocturnal wake time. Future research could investigate ways to improve dyadic perceptions of partner nocturnal wake time and examine how parents' understanding of their partners' sleep could be used to negotiate nocturnal caregiving responsibilities. For example, one parent could be designated to wake up at night with the infant and thus suffer from 
sleep fragmentation. Whereas, the other parent could be designated to stay up late, or wake up early with the infant and thus suffer from partial sleep deprivation. An informed and negotiated approach to nocturnal caregiving could provide families with the ability to maximize rest within the dyad.

\section{Research Question 5: Summary}

The current findings demonstrate that the accuracy of mothers' and fathers' reports of their own nocturnal wake time were significantly different, in that fathers' reports were closer to objective measures than mothers' reports. Additionally, the accuracy of mothers' and fathers' reports of their partners' nocturnal wake time did not significantly differ; however, fathers' reports were closer to mothers' objective measures than mothers' reports were to fathers' objective measures. Again, clinical significance of these findings can not be determined from the current reports, however these reports provide an initial description of parental perception of their nocturnal sleep. Similar to the summary of the previous research question, the current findings help to better understand dynamic family interactions among new parents. Parents may perceive their own sleepiness or fatigue based on how they think they slept rather than how they actually slept. For example previous research has indicated that perception of sleep disturbance among new mothers is indicative of daytime behaviors, such as napping (Cottrell \& Karraker, 2002). Future research investigations of parental perceptions of their own, and their partners' sleep could be beneficial to new families. A more accurate understanding of one's own and one's partner's nocturnal wake time could help promote a more informed approach to nocturnal caregiving efforts or obtaining rest among new families. 


\section{Limitations and Future Directions}

The current study had two primary research objectives. The research objectives were empirically explored, though not without limitations that included sampling, measurement and analyses, and research design issues.

\section{Sampling Limitations}

Efforts were made to acquire a representative sample of first-time postpartum parents from the north-central West Virginia region. According to the United States Census data (2008) ethnicity was approximately matched among the current sample (92.86\% White-Non Hispanic), and West Virginia (93.5\% White-Non Hispanic), but not the United States (65.6\% White-Non Hispanic). Household income was approximately $\$ 20,000$ and $\$ 6,000$ higher, respectively, among the current sample (\$56,091) compared to West Virginia $(\$ 37,057)$, and the United States $(\$ 50,740)$. Education among the current sample $(M=15.5$ years of education $)$ appeared higher than that among West Virginians (14.8\% bachelor's degree or higher) and among the United States (24.4\% bachelor's degree or higher).

The community-based recruitment strategies appeared successful in obtaining a study sample similar to the West Virginia population. However, as with any social and behavioral research, sampling bias may have occurred as exemplified by the higher income and education among the current sample. More affluent, or educated parents may have been more willing to participate in the research protocol; furthermore, previous research indicates that better sleep is interrelated with a network of variables that include higher income, higher education, and better mental and physical health (Moore, Adler, Williams, \& Jackson, 2002). Additionally, due to a low ethnic diversity among the current sample, it is inconclusive whether these results would generalize to postpartum parents of ethnic groups other than White-Non-Hispanic. Specifically, 
bed sharing is known to differ among ethnic groups (Thoman, 2006) and bed sharing may in turn influence postpartum sleep and sleepiness profiles.

\section{Measurement and Analyses Limitations}

The standard MSLT protocol calls for an overnight PSG sleep assessment the night before a MSLT. The PSG assessment enables clinicians to examine the quality and quantity of sleep on the night prior to the MSLT, and to clinically diagnose potential sleep disorders (Littner et al., 2005). The protocol for the current study did not implement an overnight PSG sleep study. However, as reported in the preliminary analyses, neither objectively measured sleep from the night prior to the MSLT, nor sleep disorder symptoms appeared to be associated with sleep onset latency. Therefore, the current methodology is considered acceptable.

Sleep stage scoring is a process typically performed by registered technicians. However, the current data scoring among two separate data analysts was deemed reliable.

The current examination of sleep and sleepiness values among postpartum couples yielded dyadic data. The current analyses included repeated measures ANOVAs to control for the dependency of the data within each dyad. This statistical method was previously used to examine sleep variables among postpartum couples (Gay et al., 2004). However, an actor-partner dyadic data analysis would be more appropriate to control for the interdependency of the data within each dyad (Kelley \& Thibaut, 1978). Actor-partner dyadic data analyses will be an important next step for examination of these data.

\section{Study Design Limitations}

The current study design would be strengthened by an assessment of nulliparous control couples that could be compared to the postpartum couples. Currently a control group of 
nulliparous couples are being recruited and investigated via the same protocol in order to build upon the current study and strengthen the current work.

Future research should build upon the current study through examination of: sleep and sleepiness among multiparous postpartum couples; various infant outcomes that may result from parental sleep and sleepiness; different mechanisms that may lead to sleep and sleepiness; the effect that mood disturbances and psychological factors may play on postpartum sleep and sleepiness, and a longitudinal sequence that may include multiple time points of measurement such as during pregnancy, and throughout earlier and later periods throughout the postpartum period.

\section{Conclusions}

The current study yielded novel findings that will add to the postpartum sleep literature and reveal new directions for future investigations.

The investigation of sleep and sleepiness among postpartum parents identified that: 1) mothers had more nocturnal and 24-hour sleep time than fathers; however, mothers' sleep was highly fragmented; 2) sleep profiles that lead to sleepiness among postpartum couples remains unknown; 3) fathers appear to have higher levels of sleepiness than postpartum mothers, though both mothers and fathers have high levels of sleepiness.

The investigation of parents' perceptions of their own and their partner's sleep, and sleepiness identified that: 1) fathers are better at reporting their partner's sleepiness than are mothers; and 2) fathers appear to be better judges of their own nocturnal wake time than are mothers.

The current study expands the knowledge of the relatively unknown sleep and sleepiness experience of postpartum fathers. Furthermore, the current study is the first to objectively report 
sleep and sleepiness values among postpartum mothers and fathers during their early postpartum period. The early postpartum period is important in the context of these findings because it is a time when one, or both, parents go back to work to function as productive members of society and they have a new infant to care for, yet new parents may experience various sleep and sleepiness-associated impairments.

The current study revealed novel results that may be used to reconsider the current FMLA policy (1998). The results from this study should be used to improve the functioning and wellbeing among new families via the development of appropriate postpartum sleep interventions, as well as stimulate empirical investigations of sleep, sleepiness, and safety within the family context. 


\section{References}

A Better Balance (2010). Retrieved January11, 2010, from http://abetterbalance.org/cms/ index.php?option $=$ com_content\&task $=$ view\&id=29\&Itemid $=49$

Acebo, C., \& LeBourgeois, M. K. (2006). Actigraphy. Respiratory Care Clinics of North America, 12, 23-30.

Afifi, L., Kushida, C., A., \& Carskadon, M. A. (2005). Multiple sleep latency test. In C. A. Kushida (pp. 11-24). Sleep deprivation: clinical issues, pharmacology, and sleep loss effects. New York: NY, Marcel Dekker.

Altman, D. G., \& Bland, J. M. (1983). Measurement in medicine: the analysis of method comparison studies. The Statistician, 32, 307-317.

Alvarez, G. G. \& Ayas, N. T. (2004). The impact of daily sleep duration on health: A review of the literature. Progress in Cardiovascular Nursing, 19, 56-59.

American Academy of Sleep Medicine. (2007). The AASM Manual for the Scoring of Sleep and Associated Events. Westchester, IL: Author.

Arand, D., Bonnet, M., Hurwitz, T., Mitler, M., Rosa, R., \& Sangal, B. R. (2005). The clinical use of the MSLT and MWT. Sleep, 28, 123-144.

Armstrong, K. L., Van Haeringen, A. R., Dadds, M. R., \& Cash, R. (1998). Sleep Deprivation or Postnatal Depression in Later Infancy: Separating the chicken from the egg. Journal of Pediatrics and Child Health, 34, 260-262.

Barrett, L. F., \& Barrett, D. J. (2001). An introduction to computerized experience sampling in psychology. Social Science Computer Review, 19, 175-185. 
Benbadis, S. R. (2005). Questionnaires and rating scales. In. C. A. Kushida (Ed.) Lung biology in health and disease: Vol. 193, pp. 1-9, Sleep deprivation: Clinical Issues, Pharmacology, and Sleep Loss Effects. New York, NY: Marcel Dekker.

Bonnet, M. H. (1985). Effect of sleep disruption on sleep, performance, and mood. Sleep, 8, 11 19.

Bonnet, M. H. (1986). Performance and sleepiness as a function of frequency and placement of sleep disruption. Psychophysiology, 23, 263-271.

Bonnet, M. H., Balkin, T. J., Dinges, D. F., Roehrs, T., Rogers, N. L., \& Wesensten, N. J. (2005). The use of stimulants to modify performance during sleep loss: A review by the sleep deprivation and stimulant task force of the American Academy of Sleep Medicine. Sleep, 28, 1163-1187.

Cabrera, N. J.,Tamis-LeMonda, C. S., Bradley, R. H., Hofferth, S., \& Lamb, M. E. (2000). Fatherhood in the twenty-first century. Child Development, 71, 127-136.

Carskadon, M. A. \& Dement, W. C. (1975). Sleep tendency; an objective measure of sleep loss. Sleep Research, 6, 200.

Carskadon, M. A., \& Dement, W. C. (1981). Cumulative effects of sleep restriction on daytime sleepiness, Psychophysiology, 18, 107-113.

Carskadon, M. A. Dement, W. C., Mitler, M. M., Roth, T., Westbrook, P. R., \& Keenan, S. (1986). Guidelines of the Multiple Sleep Latency Test (MSLT): a standard measure of sleepiness. Sleep, 9, 519-525. 
Carskadon, M. A., \& Rechtschaffen, A. (2005). Monitoring and staging human sleep. In Principles and practice of sleep medicine. In: M. H. Kryger, T. Roth, \& W. C. Dement (Eds.) Principles and practice of seep medicine. (pp. 1359-1377) ( $4^{\text {th }}$ ed.). Philadelphia, Pa: Elsevier Saunders.

Condone, J. T., Boyce, P., Corkindale, C. J. (2004). The first-time fathers study: a prospective study of the mental health and wellbeing of men during the transition to parenthood. Australian and New Zealand Journal of Psychiatry, 38, 56-64.

Copinschi, G. (2005). Metabolic and endocrine effects of sleep deprivation. Essential Psychopharmacology, 6, 341-347.

Dads ‘sleep on' while babies cry. (2004, September 9). BBC News. Retrieved April 2, 2008, from http://newsvote.bbc.co.uk/mpapps/pagetools/print/news.bbc.co.uk/1/hi/health/ 3638756.stm.

Dement, W. C., \& Vaughan, C. (1999). The Promise of Sleep. New York, NY: Dell Publishing. Denenberg, V. H. (1976). Statistics and Experimental Design for Behavioral and Biological Researchers. Washington, DC: Hemisphere Publishing Corporation.

Dennis, C. L. \& Ross, L. (2005). Relationships among infant sleep patterns, maternal fatigue and development of depressive symptomology. Birth, 32, 187-193.

de Souza, L., Benedito-Silva, A.A., Pires, M.L., Povares, D., Tufik, S., \& Calil, H.M. (2003). Further validation of actigraphy for sleep studies. Sleep, 26, 81-85.

Dinges, D. F. (2005). Sleep deprivation / restriction: Effects on human cognitive performance and safety. In M. R. Opp (Ed), SRS Basics of Sleep Guide (pp. 45-53). Westchester, Illinois: Sleep Research Society. 
Dinges, D. F., Pack, F., Williams, K., Gillen, K. A., Powell, J. W., Ott, G. E., et al. (1997). Cumulative sleepiness, mood disturbance, and psychomotor vigilance performance decrements during a week of sleep restricted to 4-5 hours per night. Sleep 20, 267-277.

Drake, C. L., Roehrs, T. A., Burduvali, E., Bonahoom, A., Rosekind, M., \& Roth, T. (2001). Effects of rapid versus slow accumulation of eight hours of sleep loss. Psychophysiology, 38, 979-987.

Drummond, S. P., \& Orff, M. A. (2005). Sleep Deprivation/Restriction: Human Studies. In M. R. Opp (Ed), SRS Basics of Sleep Guide (pp. 39-43). Westchester, Illinois: Sleep Research Society.

Durmer, J. S., \& Dinges, D. F. (2005). Neurocognitive consequences of sleep deprivation. Seminars in neurology, 25, 117-129.

Elek, S. M., Hudson, D. B., \& Bouffard, C. (2003). Marital and parenting satisfaction and infant care self-efficacy during the transition to parenthood: the effect of infant sex. Issues in Comprehensive Pediatric Nursing, 26, 45-57.

Elek, S. M., Hudson, D. B., \& Fleck, M. O. (2002). Couples' experiences with fatigue during the transition to parenthood. Journal of Family Nursing, 8, 221-240.

Fagerskiold, A. (2008). A change in life as experienced by first-time fathers. Scandinavian journal of caring sciences, 22, 64-71.

Faul, F., \& Erdfelder, E. (1992). GPOWER: A priori, post-hoc, and compromise power analyses for MS-DOS [Computer program]. Bonn, FRG: Bonn University, Department of Psychology.

Ferrara, M., \& De Gennaro, L. (2002). How much sleep do we need? Sleep Medicine Reviews, 5, $155-179$. 
Gallup Organization (2003). National survey of distracted and drowsy driving attitudes and behaviors. Washington: National Highway Traffic Safety Administration.

Gay, C. L., Lee, K. A., \& Lee, S. Y. (2004). Sleep patterns and fatigue in new mothers and fathers. Biological Research for Nursing, 5, 311-318.

Gaylor, E., \& Manber, R. (2005). Pregnancy and postpartum. In C. A. Kushida (Ed.) Lung biology in health and disease: Vol. 193, pp. 177-194, Sleep deprivation: Clinical Issues, Pharmacology, and Sleep Loss Effects. New York, NY: Marcel Dekker.

Harrison, Y. \& Horne, J. A. (2000). The impact of sleep deprivation on decision making: A review. Journal of Experimental Psychology: Applied, 6, 236-249.

Hisock, H. \& Wake, M. (2001). Infant sleep problems and postnatal depression: A community based sample. Pediatrics, 107, 1317-1322.

Hisock, H. \& Wake, M. (2002). Randomised controlled trial of behavioural infant sleep intervention to improve infant sleep and maternal mood. British Medical Journal, 324, 1062-1067.

Howell, D. C. (2002). Statistical methods for psychology ( $5^{\text {th }}$ ed.). Belmont, CA: Duxbury Press.

Insana, S. P., \& Montgomery-Downs, H. E. (2007). Postpartum sleep disturbance and psychomotor vigilance performance. Sleep: Abstract Supplement, 30, A323.

Insana, S. P., \& Montgomery-Downs, H. E. (2009). Body Over Mind: Predictors of Daytime Sleepiness among Postpartum Mothers. Sleep: Abstract Supplement, 32, A137-A138.

Jean-Louis, G., von Gizycki, H., Zizi, F., Fookson, J., Spielman, A., Nunes, J., Fullilove, R., \& Taub, H. (1996). Determination of sleep and wakefulness with the actigraph data analysis software (ADAS). Sleep, 19, 739-743. 
Jenni, O. G., \& Carskadon, M. A. (2005). Sleep Deprivation/Restriction: Human Studies. In M. R. Opp (Ed), SRS Basics of Sleep Guide (pp. 11-19). Westchester, Illinois: Sleep Research Society.

Jones, L.C., \& Heermann, J. A. (1992). Parental division of infant care: contextual influences and infant characteristics. Nursing Research, 41, 228-234.

Karacan, I., Williams, R. L., Hursch, C. J., McCaulley, M., Heine, M. W. (1969). Some implications of the sleep aptterns of pregnancy for postpartum emotional disturbances. The British Journal of Psychiatry, 115, 929-935.

Karraker, K. H. \& Young, M. (2007). Night waking in 6-month-old infants and maternal depressive symptoms. Journal of applied developmental psychology, 28, 493-498.

Kamerman, S. B. (2000). From maternity to parental leave policies: women's health, employment, and child and family well-being. Journal of the American Women's Association, 55, 96-99.

Kang, M. J., Matsumoto, K., Shinkoda, H., Mishima, M., \& Seo, Y. J. (2002). Longitudinal Study for Sleep-Wake Behaviours of Mothers from Pre-Partum to Post-Partum Using Actigraph and Sleep Logs. Psychiatry and Clinical Neurosciences, 56, 251-252.

Kelley, H. H., \& Thibaut, J. W. (1978). Interpersonal Relations. New York: John Wiley \& Sons.

Kennedy, H. P., Gardiner, A., Gay, C., \& Lee, K. A. (2007). Negotiating sleep: a qualitative study of new mothers. The Journal of Perinatal and Neonatal Nursing, 21, 114-122

Keppel, G. \& Wickens, T. D. (2004). Design and Analysis: A researcher's handbook $4^{\text {th }}$ ed. Upper Saddle River, NJ: Prentice Hall. 
Kushida, C.A., Chang, A., Gadkary, C., Guilleminault, C., Carrillo, O., \& Dement, W.C. (2001). Comparison of actigraphic, polysomnographic, and subjective assessment of sleep parameters in sleep-disordered patients. Sleep Medicine, 2, 389-396.

Laflamme, D., Pomerleau, A., \& Malcuit, G. (2002). A comparison of fathers' and mothers' involvement in childcare and stimulation behaviors during free-play with their infants at 9 and 15 months. Sex Roles, 47, 507-518.

Lamb, M. E., Bornstein, M. H., \& Teti, D. M. (2002). Development in infancy: An introduction ( $4^{\text {th }}$ edition). Mahwah, NJ: Erlbaum.

Lee, K. A. (1992). Self-reported sleep disturbances in employed women. Sleep, 15, 493-498.

Lee, K. A., McEnany, G., \& Zaffke, M. E. (2000). REM sleep and mood state in childbearing women: sleepy or weepy? Sleep, 23, 1-9.

Levine, B., Roehrs, T., Stepanski, E., Zorick,F., \& Roth, T. (1987). Fragmenting sleep diminishes its recuperative value. Sleep, 10, 590-599.

Littner, M. R., Kushida, C., Wise, M., Davila, D. G., Morgenthaler, T., Lee-Chiong, T., et al. (2005). Practice parameters for clinical use of the Multiple Seep Latency Test and the Maintenance of Wakefulness Test. Sleep, 28, 113-121.

Lumley, M., Roehrs, T., Asker, D., Zorick, F., \& Roth, T. (1987). Ethanol and caffeine effects on daytime sleepiness / alertness. Sleep, 10, 306-312.

Manber, R. \& Armitage, R. (1999). Sex, Steroids, and Sleep: A Review. Sleep, 22, 540-555.

Martin, S. E., Engleman, H. M., Deary, I. J., \& Douglas, N. J. (1996). The effect of sleep fragmentation on daytime function. American Journal of Respiratory and Critical Care Medicine, 153, 1328-1332. 
Matthey, S., Barnett, B., Kavanagh, D. J., Howie, P. (2001). Validation of the Edinburgh Postnatal Depression Scale for men, and comparison of item endorsement with their partners. Journal of Affective Disorders, 64, 175-84.

Meijer, A. M., \& vandenWittenboer, G. L. (2007). Contribution of infants' sleep and crying to marital relationship of first-time parent couples in the $1^{\text {st }}$ year after childbirth. Journal of Family Psychology, 21, 49-57.

Moore, P. J., Adler, N. E., Williams, D. R., \& Jackson, J. S. (2002). Socioeconomic status and health: the role of sleep. Psychosomatic Medicine, 64, 337-344.

Murray, L., Cooper, P., \& Hipwell, A. (2003). Mental health of parents caring for infants. Archives of Women's Mental Health, 6, 71-77.

Nepomnyaschy, L., \& Waldfogel, J. (2007). Paternity leave and fathers' involvement with their young children: Evidence from the American ECLS-B. Community, Work \& Family, 10, 427-453.

Nishihara, K., Horiuchi, S., Eto, H., \& Uchida, S. (2001). Comparisons of sleep patterns between mothers in post-partum from 9 to 12 weeks and non-pregnant women. Psychiatry and Clinical Neurosciences, 55, 227-228.

Nystrom, K., \& Ohrling, K. (2004). Parenthood experiences during the child's first year: literature review. Journal of Advanced Nursing, 46, 319-330.

Ohayon, M. M., Carskadon, M. A., Guilleminault, C., \& Vitiello, M. V. (2004). Meta-analysis of quantitative sleep parameters from childhood to old age in healthy individuals: Developing normative sleep values across the human lifespan. Sleep, 27, 1255-1273. 
Paulson, J. F., Dauber, S., \& Leiferman, J. A. (2006). Individual and combined effects of postpartum depression in mothers and fathers on parenting behavior. Pediatrics, 118, 659-668.

Premberg, A., Hellstrom, A. L., \& Berg, M. (2008). Experiences of the first year as a father. Scandinavian journal of caring sciences, 22, 56-63.

Radloff, L. A. (1977). The CES-D scale: A self-report depression scale for research in the general population. Applied Psychological Measurement, 1, 385-401.

Righetti-Veltema, M., Conne-Perreard, E., Bousquet, A., \& Manzano, J. (2002). Postpartum depression and mother-infant relationship at 3 months old. Journal of Affective Disorders, 70, 291-306.

Roehrs, T., Carskadon, M. A., Dement, W. C., \& Roth, T. Daytime Sleepiness and Alertness. In M. H. Kryger, T. Roth, \& W. C. Dement (Eds.), Principles and Practice of Sleep Medicine $\left(4^{\text {th }}\right.$ ed., pp. 39-50). Philadelphia, PA: Elsevier Saunders.

Rosekind, M. R. (2005). Underestimating the societal costs of impaired alertness: safety, health and productivity risks. Sleep Medicine, 6, S21-S25.

Rosenthal, L., Roehrs, T. A., Rosen, A., \& Roth, T. (1993). Level of sleepiness and total sleep time following various time in bed conditions. Sleep, 16, 226-232.

Ross, L. E., \& McLean, L. M. (2006). Anxiety disorders during pregnancy and the postpartum period: A systematic review. Journal of Clinical Psychiatry, 67, 1285-1298.

Ross, L. E. Murray, B. J., \& Steiner, M. (2005). Sleep and perinatal mood disorders: a critical review. Journal of Psychiatry and Neuroscience, 30, 247-256.

Sadeh, A., \& Acebo, C. (2003). The role of actigraphy in sleep medicine. Sleep Medicine Reviews, 6, 113-124. 
Santiago, J. R., Nolledo, M. S., Kinzler, W., Santiago, T. V. (2001). Sleep and sleep disorders in pregnancy. Annals of Internal Medicine, 134, 396-408.

Schor, E. L. (2003). Family pediatrics: report of the Task Force on the Family. Pediatrics, 111, 1541-1571.

Shinkoda, H., Matsumoto, K., \& Park, Y. M. (1999). Changes in sleep-wake cycle during the period from late pregnancy to puerperium identified through the wrist actigraph and sleep logs. Psychiatry and Clinical Neurosciences, 53, 133-135.

Smart, J. \& Hiscock, H. (2007). Early infant crying and sleeping problems: A pilot study of impact on parental well-being and parent-endorsed strategies for management. Journal of Pediatrics and Child Health, 43, 284-290.

Soliday, E., McCluskey-Fawcett, K., \& O’Brien, M. (1999). Postpartum affect and depressive symptoms in mothers and fathers. The American journal of orthopsychiatry, 69, 30-38.

Spiegel, K., Knutson, K., Leproult, R., Tasali, E., \& Van Cauter, E. (2005). Sleep loss: a novel risk factor for insulin resistance and Type 2 diabetes. Journal of Applied Physiology, 99, 2008-2019.

Staehelin, K., Bertea, P. C., \& Stutz, E. Z. (2007). Length of maternity leave and health of mother and child-a review. International Journal of Public Health, 52, 202-209.

Swain, A. M., O’Hara, M. W., Starr, K. R., \& Gorman, L. L. (1997). A prospective study of sleep, mood, and cognitive function in postpartum and nonpostpartum women. Obstetrics and Gynecology, 90, 381-386.

Tamaki, M., Nittono, H., Hayashi, M., \& Hori, T. (2005). Examination of the first-night effect during the sleep-onset period. Sleep, 28, 195-202. 
Tamakoshi, A., \& Ohno, Y. (2004). Self-reported sleep duration as a predictor of all-cause mortality: Results from the JACC study, Japan. Sleep, 27, 51-54.

Thoman, E. B. (2002). Cosleeping, and ancient practice: issues of the past and present, and possibilities for the future. Sleep medicine Reviews, 10, 407-417.

Thomas, K. A., \& Foreman, S. W. (2005). Infant sleep and feeding pattern: effects on maternal sleep. Journal of Midwifery \& Women's health, 50, 399-404.

Thorpy, M. J. (Ed.). (2003). SleepMultiMedia: The Computerized Textbook. Scarsdale, N.Y.: Sleep Multimedia Inc.

Thorpy, M. J. (1992). The clinical use of the Multiple Sleep Latency Test. The standards of practice committee of the American Sleep Disorders Association. Sleep, 15, 268-276.

United States Department of Labor (1993). The Family and Medical Leave Act of 1993. Retrieved April 24, 2008, from http://www.dol.gov/dol/allcfr/ESA/Title_29/Part_825/ toc.htm

United States Census Bureau (2008). State and Country Quick Facts: West Vriginia. Retrieved January 2, 2010, from http://quickfacts.census.gov/qfd/states/54000.html

Van Dongen, H. P., Maislin, G., Mullington, J. M., \& Dinges, D. F. (2003). The cumulative cost of additional wakefulness: dose-response effects on neurobehavioral functions and sleep physiology from chronic sleep restriction and total sleep deprivation. Sleep, 26, 117-126. Vaughn, B. V. \& D’Cruz, O. F. (2005). Cardinal manifestations of sleep disorders. In M. H. Kryger, T. Roth, \& W. C. Dement (Eds.), Principles and Practice of Sleep Medicine $\left(4^{\text {th }}\right.$ ed., pp. 594-601). Philadelphia, PA: Elsevier Saunders. 
Viens, M., De Koninck, J., Mercier, P., St-Onge, M., \& Lorrain, D. (2003). Trait anxiety and sleep-onset insomnia: evaluation of treatment using anxiety management training. Journal of Psychosomatic Research, 54, 31-37.

Walsh, J. K., Dement, W. C., \& Dinges, D. F. (2005). Sleep medicine, pulbic policy, and public health. In M. H. Kryger, T. Roth, \& W. C. Dement (Eds.), Principles and Practice of Sleep Medicine (4 ${ }^{\text {th }}$ ed., pp. 648-656). Philadelphia, PA: Elsevier Saunders.

Warren, S. L., Howe, G., Simmens, S. J., \& Dahl, R. E. (2006). Maternal depressive symptoms and child sleep: models of mutual influence over time. Developmental psychopathology, $18,1-16$.

Yoo, S., Hu, P. T., Gujar, N., Jolesz, F. A., \& Walker, M. P. (2007). A deficit in the ability to form new human memories without sleep. Nature Neuroscience, 10, 385-392. 
Table 1

Pilot Data: Descriptive Statistics for Primiparious Mothers Sleep Variables during postpartum week three

\begin{tabular}{lcccc}
\hline & $M$ & $S D$ & Skew & Kurtosis \\
\hline $\begin{array}{l}24 \text { Hour } \\
\text { Total Sleep } \\
\text { Time (min.) }\end{array}$ & 455.87 & 66.27 & -1.23 & 1.72 \\
$\begin{array}{l}\text { Nocturnal } \\
\text { Sleep Time } \\
\text { (min.) }\end{array}$ & 445.56 & 67.27 & -1.50 & 2.83 \\
$\begin{array}{l}\text { Nocturnal Sleep } \\
\text { Fragmentation }\end{array}$ & & & & \\
& 21.93 & 6.56 & .85 & -.21 \\
Nap Duration & 63.79 & 47.55 & 1.76 & 4.82 \\
& & & & \\
& & & & \\
\hline $\begin{array}{l}\text { Nap Duration } \\
\text { Scored as Sleep }\end{array}$ & 61.04 & 46.17 & 1.91 & \\
\hline
\end{tabular}


Table 2

Sample Characteristics for Total Sample, Mothers, and Fathers

\begin{tabular}{|c|c|c|c|c|c|c|c|c|c|}
\hline \multirow[b]{2}{*}{ Variable } & \multicolumn{2}{|c|}{ Total $(N=42)$} & \multicolumn{2}{|c|}{ Mothers $(N=21)$} & \multicolumn{2}{|c|}{ Fathers $(N=21)$} & \multicolumn{3}{|c|}{$\begin{array}{c}\text { Paired-Sample } \\
\text { t-test }\end{array}$} \\
\hline & $M$ & $S D$ & $M$ & $S D$ & $M$ & $S D$ & $t$ & (df) & $p$ \\
\hline Age & 27.94 & 5.03 & 26.81 & 4.80 & 29.08 & 5.11 & -2.24 & $(20)$ & .04 \\
\hline Yrs. Education & 15.5 & 3.58 & 15.29 & 3.47 & 15.71 & 3.76 & -.89 & $(20)$ & .38 \\
\hline $\begin{array}{l}\text { Caffeinated } \\
\text { Drinks \# }\end{array}$ & 2.27 & 2.61 & 1.15 & 1.13 & 3.59 & 3.22 & -2.96 & $(16)$ & .01 \\
\hline \multirow[t]{2}{*}{ Income } & $\$ 56,091$ & $\$ 34,320$ & - & - & - & - & - & - & - \\
\hline & $N$ & $n$ & $\%$ & $N$ & $\%$ & $N$ & $\%$ & - & - \\
\hline $\begin{array}{l}\text { Married/Living } \\
\text { together }\end{array}$ & 42 & 42 & 100 & - & - & - & - & - & - \\
\hline White & 42 & 39 & 92.86 & 19 & 90.48 & 20 & 95.24 & - & - \\
\hline Black & 42 & 1 & 2.38 & 0 & 0 & 1 & 4.76 & - & - \\
\hline Other & 42 & 2 & 4.76 & 2 & 9.52 & 0 & 0 & - & - \\
\hline $\begin{array}{l}\text { Work Status: } \\
\text { Family leave }\end{array}$ & 42 & 13 & 30.95 & 12 & 57.14 & 1 & 4.76 & - & - \\
\hline $\begin{array}{l}\text { Work Status: } \\
\text { Fulltime }\end{array}$ & 42 & 16 & 38.10 & 1 & 4.76 & 15 & 71.43 & - & - \\
\hline $\begin{array}{l}\text { Work Status: } \\
\text { Part-time }\end{array}$ & 42 & 6 & 14.29 & 2 & 9.52 & 4 & 19.05 & - & - \\
\hline $\begin{array}{l}\text { Work Status: } \\
\text { Unemployed }\end{array}$ & 42 & 7 & 16.67 & 6 & 28.57 & 1 & 4.76 & - & - \\
\hline
\end{tabular}

(table continues) 
Table 2 (continued)

\begin{tabular}{|c|c|c|c|c|c|c|c|c|c|}
\hline & $N$ & $n$ & $\%$ & $M$ & $S D$ & - & - & - & - \\
\hline $\begin{array}{l}\text { Infant } \\
\text { Gestational Age } \\
\text { (weeks) }\end{array}$ & 21 & - & - & 39.61 & 1.09 & - & - & - & - \\
\hline $\begin{array}{l}\text { Infant Age } \\
\text { during study } \\
\text { (weeks) }\end{array}$ & 21 & - & - & 6.93 & 1.26 & - & - & - & - \\
\hline $\begin{array}{l}\text { Feeding } \\
\text { Method: } \\
\text { Breast }\end{array}$ & 21 & 13 & 61.90 & - & - & - & - & - & - \\
\hline $\begin{array}{l}\text { Feeding } \\
\text { Method: } \\
\text { Formula }\end{array}$ & 21 & 3 & 14.29 & - & - & - & - & - & - \\
\hline $\begin{array}{l}\text { Feeding } \\
\text { Method: } \\
\text { Breast \& } \\
\text { Formula }\end{array}$ & 21 & 5 & 23.81 & - & - & - & - & - & - \\
\hline
\end{tabular}

Note. Infant age is in weeks. Variables were reported for status at time of study. Four fathers had missing data for Caffeinated Drinks \# 
Table 3

Target sample relationship history: In years $(N=21)$

\begin{tabular}{lcccc}
\hline Variable & Mean & $S D$ & $N$ & $\%$ \\
\hline $\begin{array}{l}\text { Time knowing } \\
\text { each other }\end{array}$ & 6.67 & 4.85 & - & - \\
$\begin{array}{l}\text { Time dating } \\
\text { Time }\end{array}$ & 5.40 & 4.27 & - & - \\
cohabitating & 4.17 & 3.17 & - & - \\
Time married & 3.33 & 1.66 & - & - \\
Married & - & - & 15 & 71.43 \\
\hline
\end{tabular}

Note. All data referring to time are calculated based on the date of each couple's participation in the laboratory based portion of the study. 
Table 4

Constructs in study and respective instruments used to measure constructs

\begin{tabular}{lll}
\hline Construct & Instrument(s) & Source \\
\hline Demographics & Demographic Form & Appendix 3 \\
& Relationship history form & Appendix 4 \\
Sleep & Actigraphy & MiniMitter, Bend Oregon \\
& Sleep / Watch diaries & Bruner Consulting Inc. \\
& Self report of reason for nocturnal wake & Appendix 5 \\
& 10-Item sleep disorders screening survey & Thorpy, 2003 \\
& The Multiple Sleep Latency Test & Carskadon \& Dement, 1975 \\
Sleepiness & Self report of sleep onset latency & Appenxix 5 \\
& & \\
Depression & Center for Epidemiologic Studies & Radloff, 1977 \\
Symptoms & Depression Scale & \\
\hline
\end{tabular}


Table 5.

Skewness and kurtosis values for study variables.

\begin{tabular}{|c|c|c|c|c|c|c|c|c|}
\hline \multirow[b]{2}{*}{ Variable } & \multicolumn{4}{|c|}{ Mothers Report } & \multicolumn{4}{|c|}{ Fathers Report } \\
\hline & Skewness & $\mathrm{SE}$ & Kurtosis & $\mathrm{SE}$ & Skewness & SE & Kurtosis & $\mathrm{SE}$ \\
\hline $\begin{array}{l}\text { 24-Hour Sleep } \\
\text { Time }\end{array}$ & .16 & .51 & -.24 & .99 & -.21 & .51 & -.51 & .99 \\
\hline $\begin{array}{l}\text { Nocturnal Sleep } \\
\text { Time }\end{array}$ & .41 & .51 & .28 & .99 & .12 & .51 & -.55 & .99 \\
\hline $\begin{array}{l}\text { †Sleep } \\
\text { Fragmentation }\end{array}$ & -.56 & .51 & .90 & .99 & .21 & .51 & -.55 & .99 \\
\hline †Sleep Efficiency & -.81 & .51 & 1.01 & .99 & -.24 & .51 & -.49 & .99 \\
\hline $\begin{array}{l}\text { Nocturnal Wake } \\
\text { Time }\end{array}$ & 1.47 & .51 & 3.67 & .99 & .84 & .51 & .12 & .99 \\
\hline MSLT: SOL & -.26 & .50 & -.86 & .97 & 1.52 & .51 & 3.37 & .99 \\
\hline $\mathrm{S}: \mathrm{SOL}$ & .17 & .50 & -.97 & .97 & .10 & .50 & -.76 & .97 \\
\hline P: SOL & .16 & .50 & -.97 & .97 & .14 & .50 & -.66 & .97 \\
\hline $\begin{array}{l}\text { S: Sleep Onset } \\
\text { Latency Diff. }\end{array}$ & .26 & .50 & -1.00 & .97 & -1.25 & .51 & 2.47 & .99 \\
\hline $\begin{array}{l}\text { P: Sleep Onset } \\
\text { Latency Diff. }\end{array}$ & -.11 & .51 & -.66 & .99 & .30 & .50 & -.36 & .97 \\
\hline $\begin{array}{l}\text { S: Nocturnal Wake } \\
\text { Time }\end{array}$ & .58 & .50 & -.92 & .97 & 1.03 & .51 & -.24 & .99 \\
\hline $\begin{array}{l}\text { P: Nocturnal Wake } \\
\text { Time }\end{array}$ & 3.43 & .50 & 13.37 & .97 & .62 & .50 & -.49 & .97 \\
\hline $\begin{array}{l}\text { S: Nocturnal Wake } \\
\text { Time Diff. }\end{array}$ & .63 & .51 & -.56 & .99 & .05 & .52 & -.46 & 1.01 \\
\hline $\begin{array}{l}\text { P: Nocturnal Wake } \\
\text { Time Diff. }\end{array}$ & .09 & .52 & -.16 & 1.01 & .24 & .51 & -.98 & .99 \\
\hline
\end{tabular}

Note. $\mathrm{S}=$ Report on self. $\mathrm{P}=$ Report on partner. Diff = difference with objective measure. 
Table 6

Descriptive statistics for, and difference statistics among, mothers' and fathers' sleep and sleepiness values

\begin{tabular}{|c|c|c|c|c|c|c|c|c|c|c|}
\hline \multirow[b]{2}{*}{ Variable } & \multicolumn{3}{|c|}{ Mothers } & \multicolumn{3}{|c|}{ Fathers } & \multicolumn{4}{|c|}{ Difference Statistic } \\
\hline & $M$ & $S D$ & $S E$ & $M$ & $S D$ & $S E$ & $F$ & $d f$ & $p$ & $d$ \\
\hline 24-Hour Sleep Time & 424.83 & 42.00 & 9.23 & 375.14 & 34.09 & 7.68 & 17.31 & 1,18 & .001 & 1.30 \\
\hline Nocturnal Sleep Time & 412.76 & 39.30 & 8.76 & 366.72 & 37.34 & 8.28 & 18.06 & 1,18 & $<.001$ & 1.43 \\
\hline$\uparrow$ Sleep Fragmentation & 18.86 & 3.34 & 0.73 & 14.34 & 4.62 & 1.01 & 13.17 & 1,18 & .002 & 1.12 \\
\hline †Sleep Efficiency & 77.84 & 6.12 & 1.33 & 79.55 & 6.94 & 1.56 & 1.11 & 1,18 & .31 & 0.26 \\
\hline Nocturnal Wake Time & 88.32 & 29.62 & 6.60 & 55.63 & 25.04 & 5.59 & 21.41 & 1,18 & $<.001$ & 1.19 \\
\hline MSLT: SOL & 11.80 & 4.60 & 1.00 & 8.03 & 3.92 & 0.88 & 11.85 & 1,19 & .003 & 0.88 \\
\hline Report on Self SOL & 12.08 & 3.86 & 0.84 & 10.76 & 4.23 & 0.92 & 1.46 & 1,20 & .24 & 0.33 \\
\hline Report on Partner SOL & 11.41 & 3.82 & 0.83 & 11.00 & 4.02 & 0.88 & 0.17 & 1,20 & .68 & 0.10 \\
\hline
\end{tabular}

Note. All variables are represented in minutes unless noted otherwise. $\dagger=$ reported as an index. $\downarrow=$ reported as a percent. Difference Statistic is reported as a repeated measures analysis of variance. 
Table 7

Difference among sleep variables from week prior, and night before MSLT: Correlations among differences and sleep onset latency

\begin{tabular}{|c|c|c|c|c|c|c|c|c|c|c|c|c|}
\hline \multirow[b]{2}{*}{ Mother: Variable } & \multicolumn{2}{|c|}{$\begin{array}{l}\text { Week Prior to } \\
\text { MSLT }\end{array}$} & \multicolumn{2}{|c|}{$\begin{array}{l}\text { Night Prior to } \\
\text { MSLT }\end{array}$} & \multicolumn{4}{|c|}{ Difference Statistic } & \multicolumn{2}{|c|}{$\begin{array}{c}\text { Difference } \\
\text { Among Variables }\end{array}$} & \multicolumn{2}{|c|}{$\begin{array}{l}\text { Differences and } \\
\text { SOL }\end{array}$} \\
\hline & $M$ & $S E$ & $M$ & $S E$ & $F$ & $d f$ & $p$ & $d$ & $M$ & $S D$ & $r$ & $p$ \\
\hline 24-Hour Sleep Time & 433.31 & 9.11 & 377.09 & 18.67 & 11.43 & 1,19 & .003 & 0.86 & 56.22 & 74.37 & -.18 & .44 \\
\hline Nocturnal Sleep Time & 423.14 & 8.26 & 357.80 & 17.63 & 21.15 & 1,19 & $<.001$ & 1.06 & 65.34 & 63.54 & -.17 & .47 \\
\hline$†$ Sleep Fragmentation & 18.99 & 0.78 & 18.21 & 1.54 & 0.23 & 1,19 & .64 & 0.14 & 0.78 & 7.32 & -.13 & .60 \\
\hline †Sleep Efficiency & 77.65 & 1.41 & 78.66 & 1.49 & 0.44 & 1,19 & .52 & 0.16 & -1.01 & 6.84 & .01 & .98 \\
\hline Nocturnal Wake Time & 87.64 & 5.68 & 69.41 & 9.16 & 2.55 & 1,18 & .13 & 0.55 & 18.24 & 49.76 & -.09 & .72 \\
\hline Father: Variable & $M$ & $S E$ & $M$ & $S E$ & $F$ & $d f$ & $p$ & $d$ & $M$ & $S D$ & $r$ & $p$ \\
\hline 24-Hour Sleep Time & 378.47 & 9.04 & 338.20 & 18.07 & 3.41 & 1,19 & .08 & 0.63 & 40.27 & 97.48 & -.02 & .93 \\
\hline Nocturnal Sleep Time & 369.88 & 8.96 & 333.49 & 17.65 & 3.58 & 1,19 & .07 & 0.58 & 36.39 & 86.06 & -.08 & .75 \\
\hline$†$ Sleep Fragmentation & 14.12 & 0.99 & 15.63 & 1.80 & 1.03 & 1,19 & .32 & 0.23 & -1.50 & 6.63 & .22 & .37 \\
\hline †Sleep Efficiency & 79.88 & 1.59 & 80.43 & 2.27 & 0.08 & 1,19 & .78 & 0.06 & -0.54 & 8.66 & .31 & .20 \\
\hline Nocturnal Wake Time & 55.25 & 6.10 & 49.20 & 6.89 & 0.58 & 1,19 & .46 & 0.21 & 6.05 & 35.64 & -.00 & .99 \\
\hline
\end{tabular}

Note. All variables are represented in minutes unless noted otherwise. $\dagger=$ reported as an index. $\downarrow=$ reported as a percent. Difference Statistic is calculated from a repeated measures analysis of variance. 
Table 8

Descriptive statistics for self-reported sleep disorder symptoms and correlations with the MSLT sleep onset latency

\begin{tabular}{|c|c|c|c|c|c|c|c|c|}
\hline \multirow[b]{2}{*}{ Variable } & \multicolumn{2}{|c|}{ Mothers } & \multicolumn{2}{|c|}{ Fathers } & \multicolumn{2}{|c|}{$\begin{array}{l}\text { Mothers sleep onset } \\
\text { latency }\end{array}$} & \multicolumn{2}{|c|}{$\begin{array}{c}\text { Fathers sleep onset } \\
\text { latency }\end{array}$} \\
\hline & $M$ & $S D$ & $M$ & $S D$ & $r$ & $p$ & $r$ & $p$ \\
\hline $\begin{array}{l}\text { Gasp, choke or stop breathing } \\
\text { during sleep }\end{array}$ & 0.10 & 0.44 & 0.29 & 0.96 & .09 & .72 & -.04 & .87 \\
\hline $\begin{array}{l}\text { Snore loudly, have high blood } \\
\text { pressure; overweight }\end{array}$ & 1.05 & 1.91 & 2.57 & 3.14 & -.03 & .90 & -.07 & .76 \\
\hline $\begin{array}{l}\text { Feel creepy crawling sensations } \\
\text { in legs while lying down }\end{array}$ & 1.00 & 2.30 & 0.86 & 1.93 & .06 & .80 & .07 & .78 \\
\hline $\begin{array}{l}\text { Feel tired and sleepy while } \\
\text { driving }\end{array}$ & 1.95 & 2.09 & 1.19 & 1.50 & -.08 & .74 & -.08 & .73 \\
\hline $\begin{array}{l}\text { Arms and legs jerk and twitch } \\
\text { during sleep }\end{array}$ & 1.76 & 3.00 & 1.43 & 1.89 & .32 & .15 & .16 & .49 \\
\hline $\begin{array}{l}\text { Wake up at night; feel fatigued } \\
\text { during the day }\end{array}$ & 5.00 & 3.08 & 2.95 & 2.89 & .16 & .48 & -.17 & .47 \\
\hline $\begin{array}{l}\text { Fall asleep in front of the TV or } \\
\text { while reading during the day or } \\
\text { early evening }\end{array}$ & 3.67 & 3.18 & 4.10 & 3.32 & .29 & .20 & -.05 & .83 \\
\hline
\end{tabular}


Table 8 (continued)

\begin{tabular}{lccccccc}
\hline & \multicolumn{2}{c}{ Mothers } & & Fathers & & $\begin{array}{c}\text { Mothers sleep onset } \\
\text { latency }\end{array}$ & $\begin{array}{c}\text { Fathers sleep onset } \\
\text { latency }\end{array}$ \\
\hline Variable & $M$ & $S D$ & $M$ & $S D$ & $r$ & $p$ & $r$ \\
\hline $\begin{array}{l}\text { Wake up tired and lethargic in } \\
\text { the morning }\end{array}$ & 3.86 & 2.90 & 4.62 & 3.04 & .41 & .07 & -.34 \\
$\begin{array}{l}\text { Have occasional sleeplessness } \\
\text { at home or during trips }\end{array}$ & 1.90 & 2.64 & 2.36 & 3.17 & .28 & .22 & -.08 \\
$\begin{array}{l}\text { Experience disturbing } \\
\text { nightmares }\end{array}$ & 1.95 & 3.02 & 2.24 & 3.91 & .23 & .32 \\
\hline
\end{tabular}

Note. The sleep disorders symptoms were rated on a scale of $0-10$ with 1 being very mild and 10 being severe. 
Table 9

Descriptive statistics for self report and report of partner on sleepiness and time awake: differences with objective measures

\begin{tabular}{lccccccccccccccccc}
\hline & \multicolumn{3}{c}{ Mothers Report } & \multicolumn{3}{c}{ Fathers Report } & \multicolumn{3}{c}{ Difference Statistics } \\
\hline \multicolumn{1}{c}{ Variable } & $M$ & $S D$ & $S E$ & $M$ & $S D$ & $S E$ & $F$ & $d f$ & $p$ & $d$ \\
\hline S: Sleep Onset Latency & 12.08 & 3.86 & .84 & 10.76 & 4.23 & .92 & 1.46 & 1,20 & .24 & 0.33 \\
S: Sleep Onset Latency Diff. & .55 & 3.78 & .85 & 3.09 & 5.07 & 5.07 & 3.78 & 1,19 & .07 & 0.57 \\
P: Sleep Onset Latency & 11.41 & 3.82 & .83 & 11.00 & 4.02 & 4.02 & .17 & 1,20 & .68 & 0.10 \\
P: Sleep Onset Latency Diff. & 3.68 & 5.01 & 1.12 & -.49 & 4.62 & 4.72 & 9.82 & 1,19 & .005 & 0.87 \\
S: Nocturnal Wake Time & 110.54 & 59.62 & 14.01 & 42.31 & 39.41 & 8.81 & 24.20 & 1,19 & $<.001$ & 1.35 \\
S: Nocturnal Wake Time Diff. & 28.95 & 55.59 & 12.74 & -10.50 & 33.96 & 7.58 & 9.66 & 1,17 & .006 & 0.86 \\
P: Nocturnal Wake Time & 39.09 & 35.44 & 7.92 & 84.84 & 46.50 & 11.40 & 17.13 & 1,19 & .001 & 1.12 \\
P: Nocturnal Wake Time Diff. & -15.17 & 31.57 & 7.16 & 1.41 & 40.06 & 9.44 & 2.82 & 1,17 & .11 & 0.46 \\
\hline
\end{tabular}

Note. $\mathrm{S}=$ Report on self. $\mathrm{P}=$ Report on partner. Diff = difference with objective measure. All variables are represented in minutes. 
Figure 1. Timeline for field and laboratory based procedures.

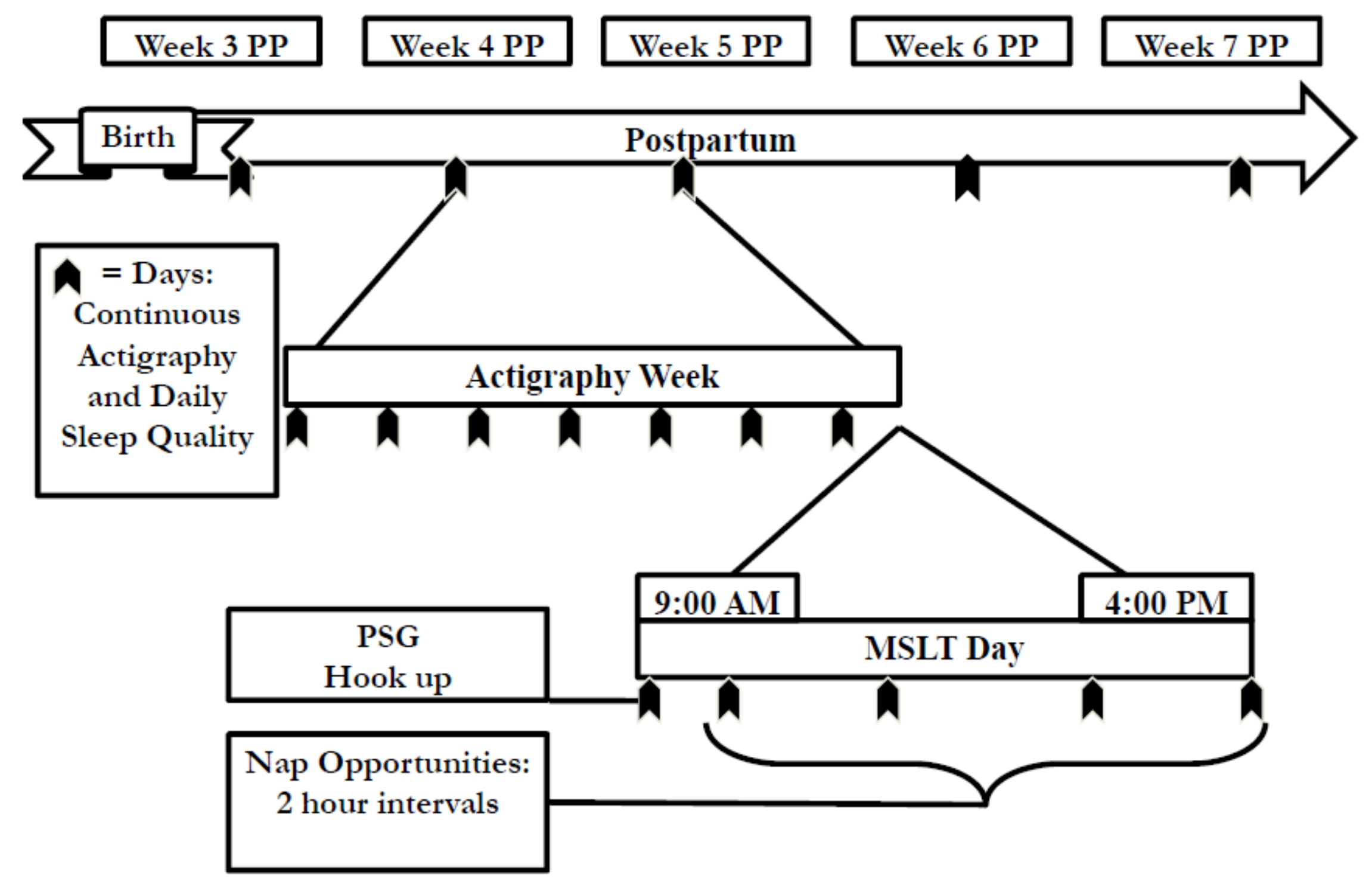


Figure 2 a. Sleep onset latency for individual mother participants: normative, moderate, and extreme sleepiness ranges identified.

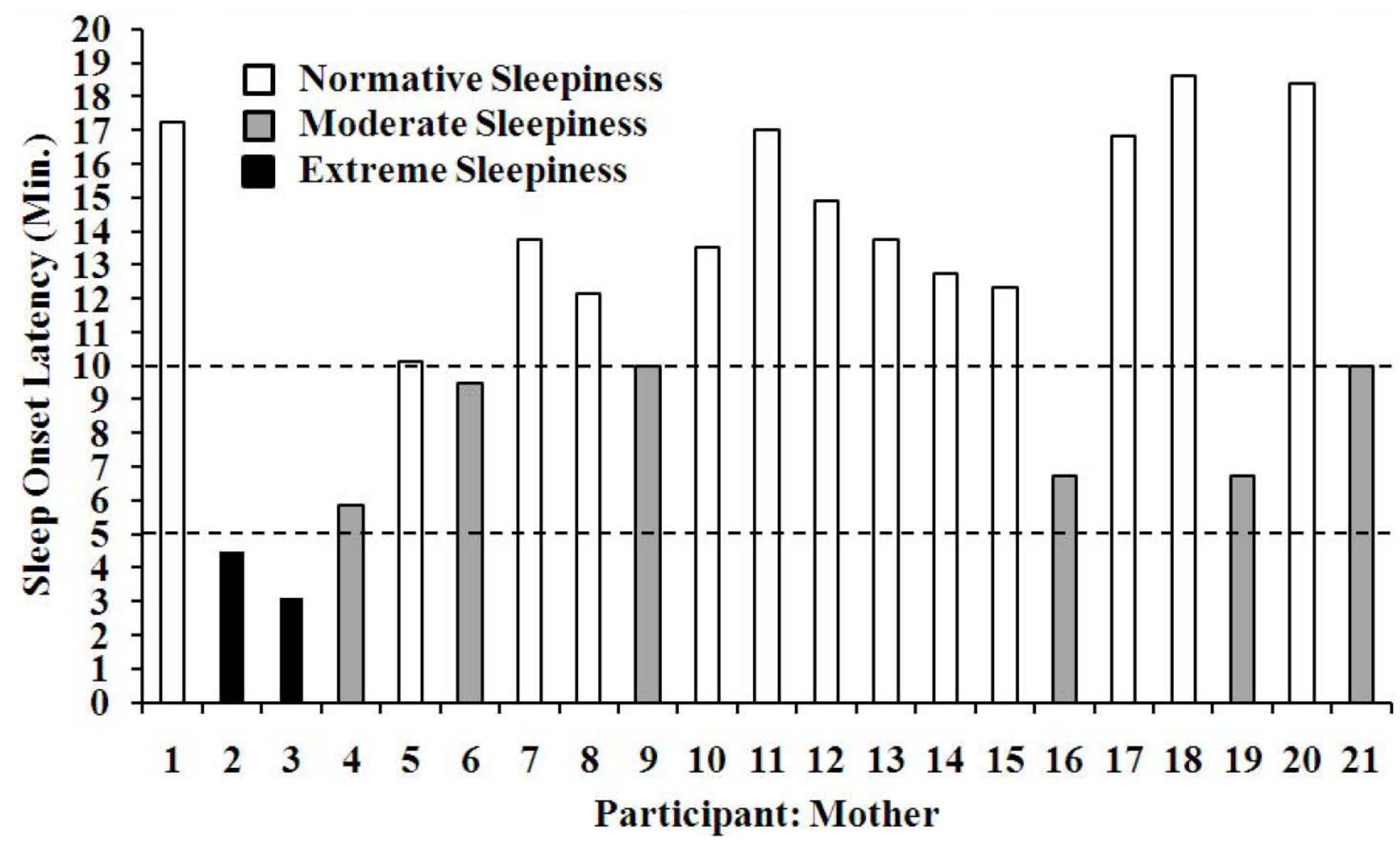


Figure $2 b$. Sleep onset latency for individual father participants: normative, moderate, and extreme sleepiness ranges identified.

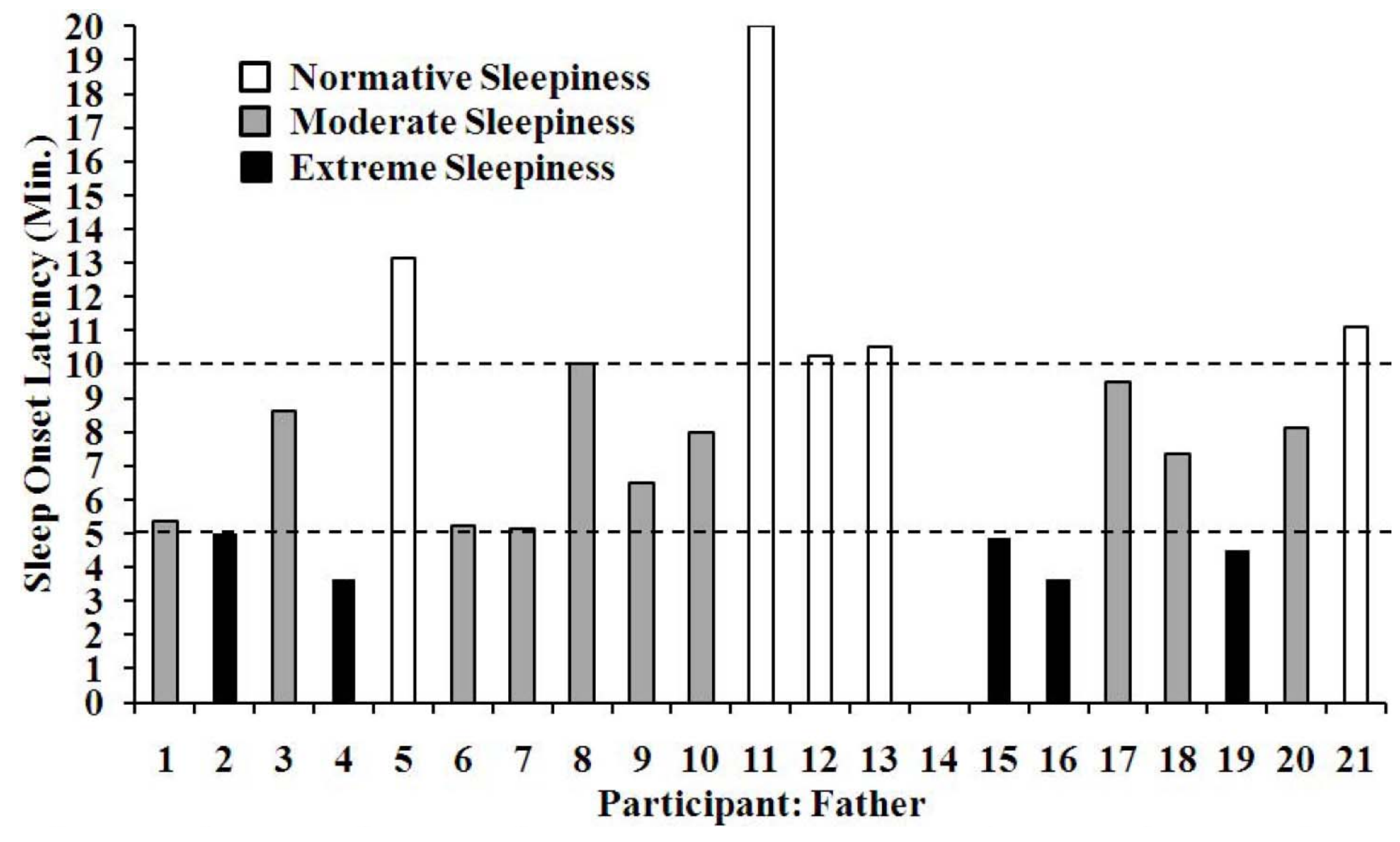


Figure 3 a. Bland-Altman plots for mothers' self-reports of sleepiness compared to mothers' objectively measured sleepiness: arbitrary guide lines at 0,25 , and 50 minute differences.

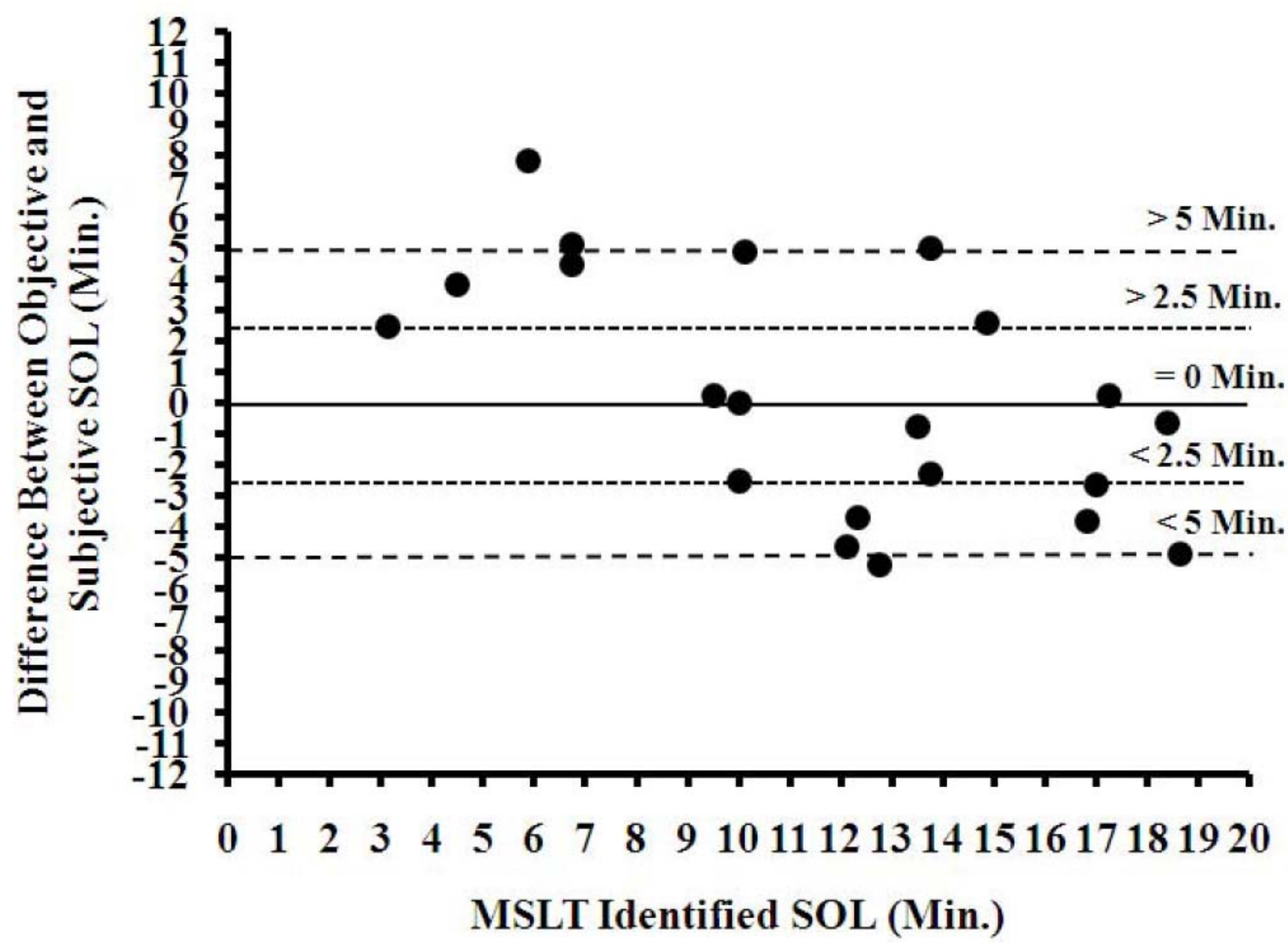


Figure 3 b. Bland-Altman plots for fathers' self-reports of sleepiness compared to fathers' objectively measured sleepiness: arbitrary guide lines at 0,25 , and 50 minute differences.

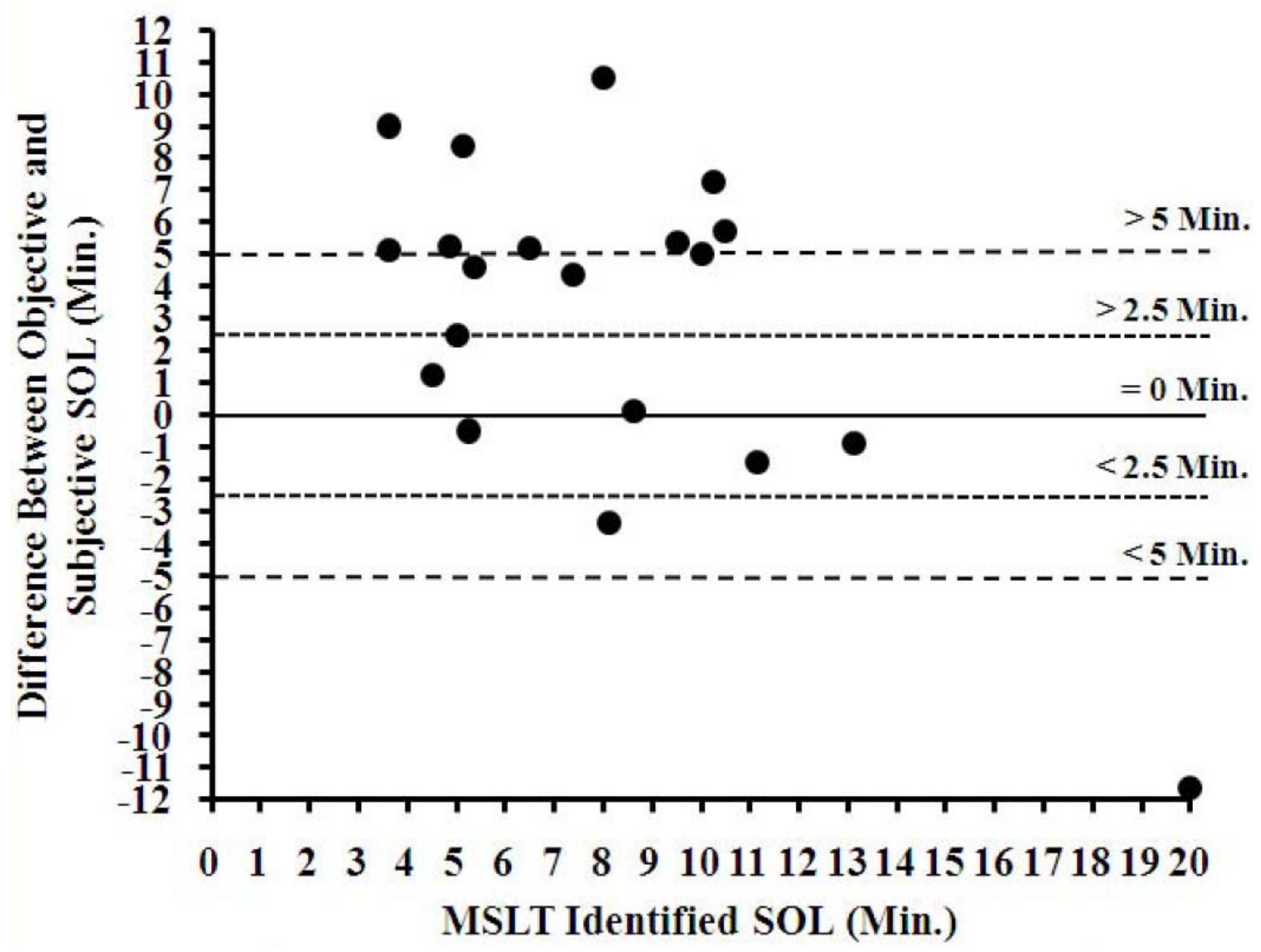


Figure 3 c. Plots for mothers' and fathers' self-reports of sleepiness compared to their objectively measured sleepiness: Means differences and standard deviations

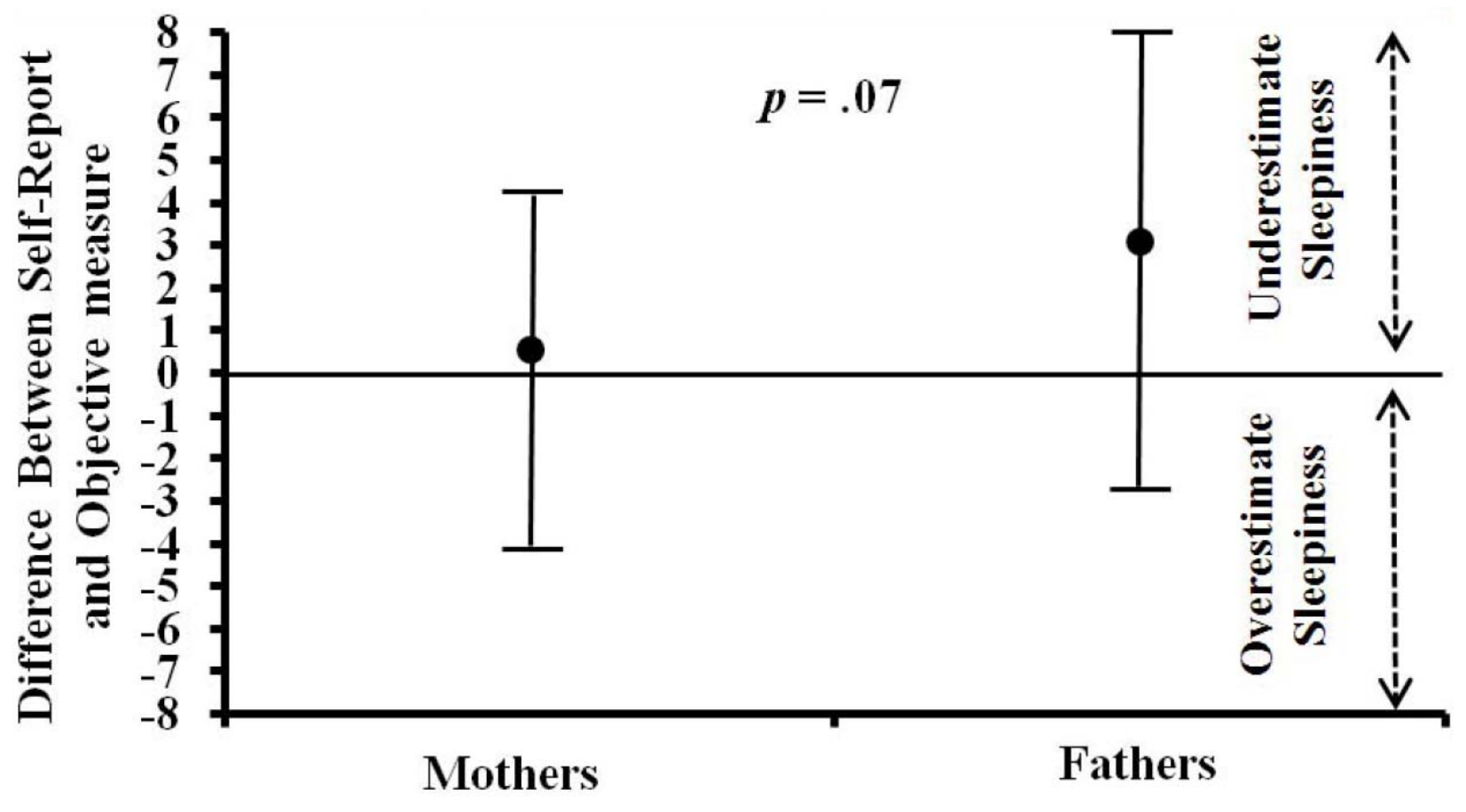

Participant 
Figure 4 a. Bland-Altman plots for mothers' reports on fathers' sleepiness when compared to father's objectively measured sleepiness: arbitrary guide lines at $0,2.5$, and 5 minute differences.

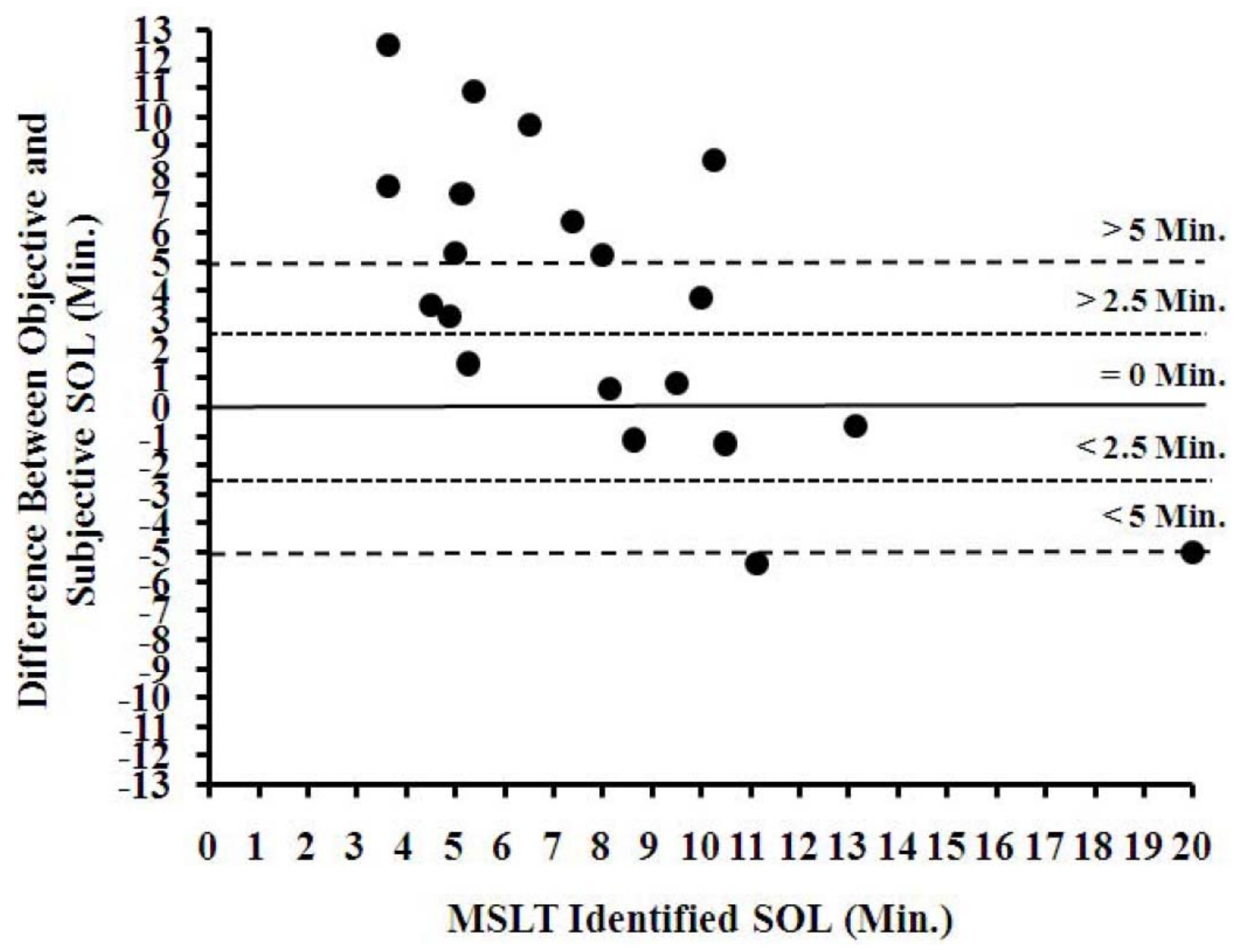


Figure $4 \mathrm{~b}$. Bland-Altman plots for fathers' reports on mothers' sleepiness when compared to mothers' objectively measured sleepiness: arbitrary guide lines at $0,2.5$, and 5 minute differences.

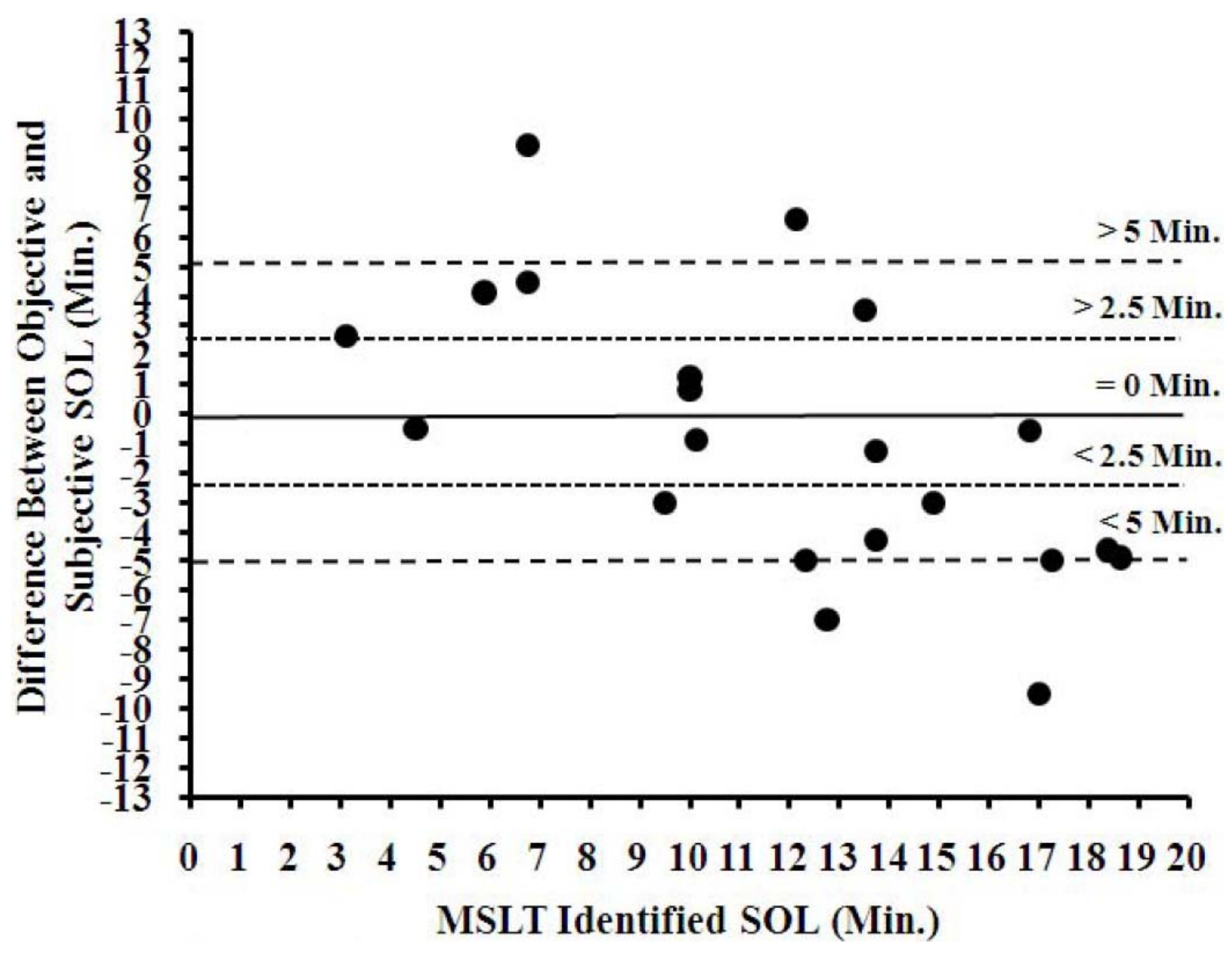


Figure $4 \mathrm{c}$. Plots for mothers' and fathers' reports on partners' sleepiness compared to their partners' objectively measured sleepiness: Means differences and standard deviations

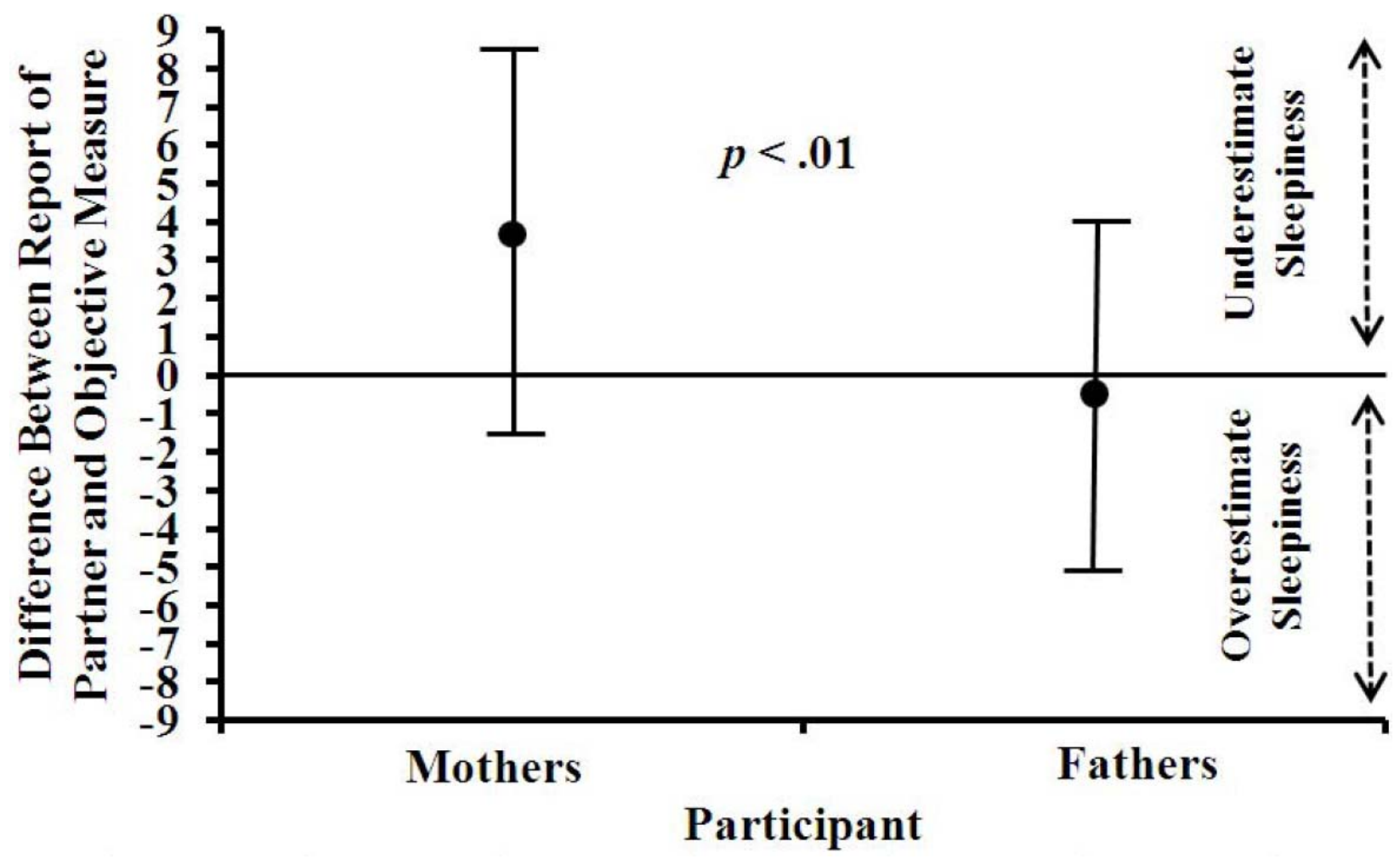


Figure 5 a. Bland-Altman plots for mothers' self-reports of nocturnal wake time compared to mothers' objectively measured nocturnal wake time: arbitrary guide lines at 0,25 , and 50 minute differences.

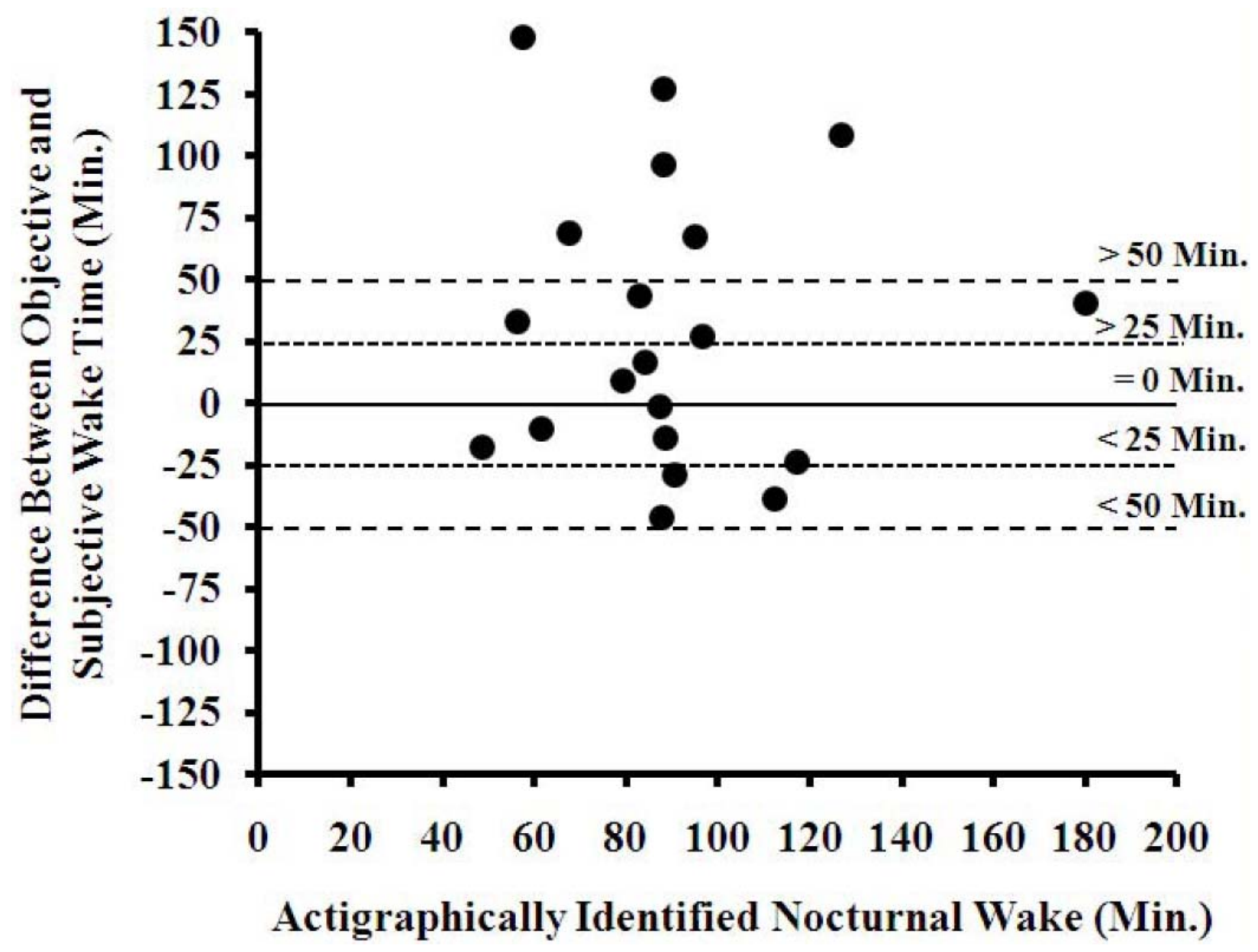


Figure $5 \mathrm{~b}$. Bland-Altman plots for fathers' self-reports of nocturnal wake time compared to fathers' objectively measured nocturnal wake time: arbitrary guide lines at 0,25 , and 50 minute differences.

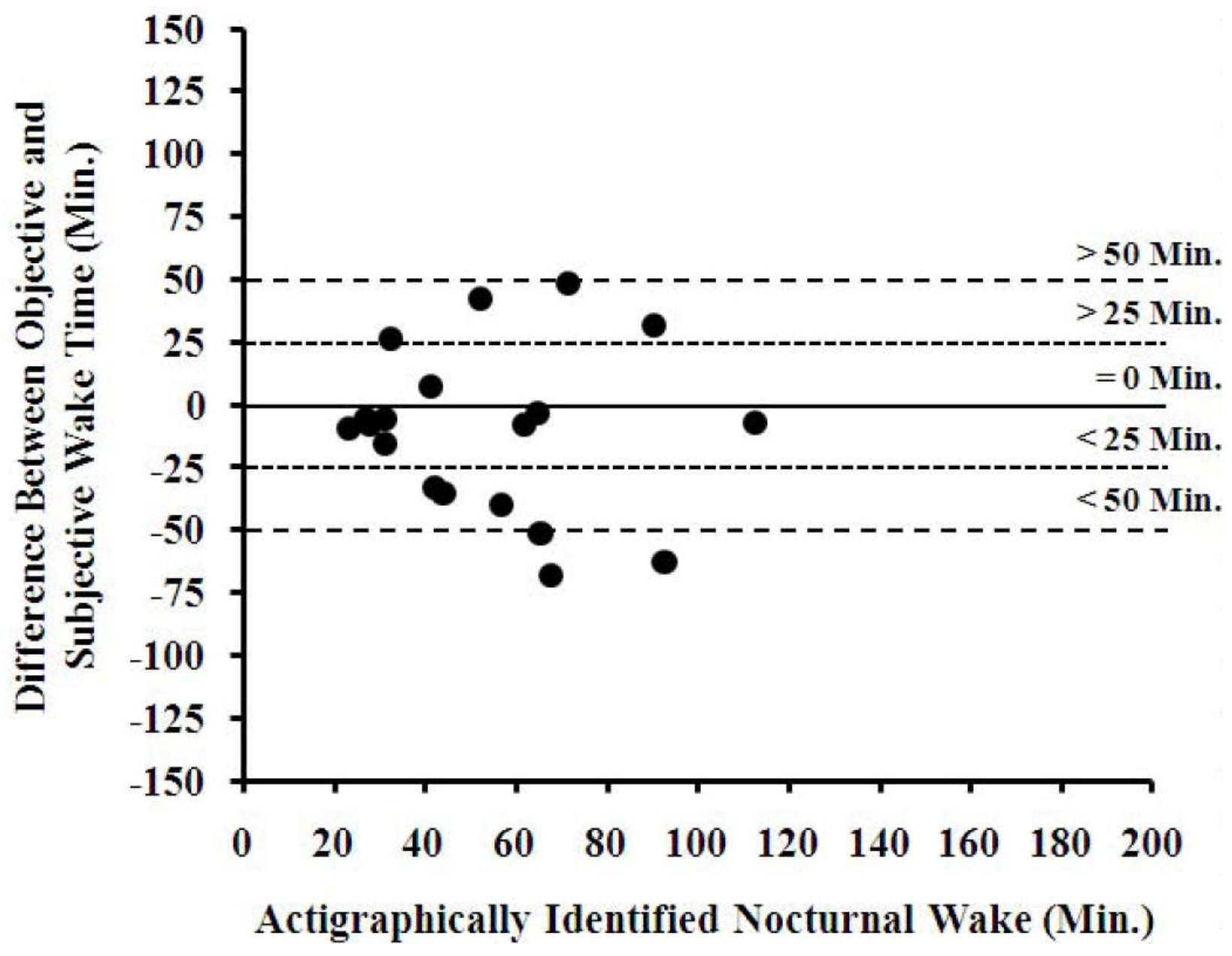


Figure 5 c. Plots for mothers' and fathers' reports on partners' nocturnal wake time compared to their partners' objectively measured nocturnal wake time: Means differences and standard deviations

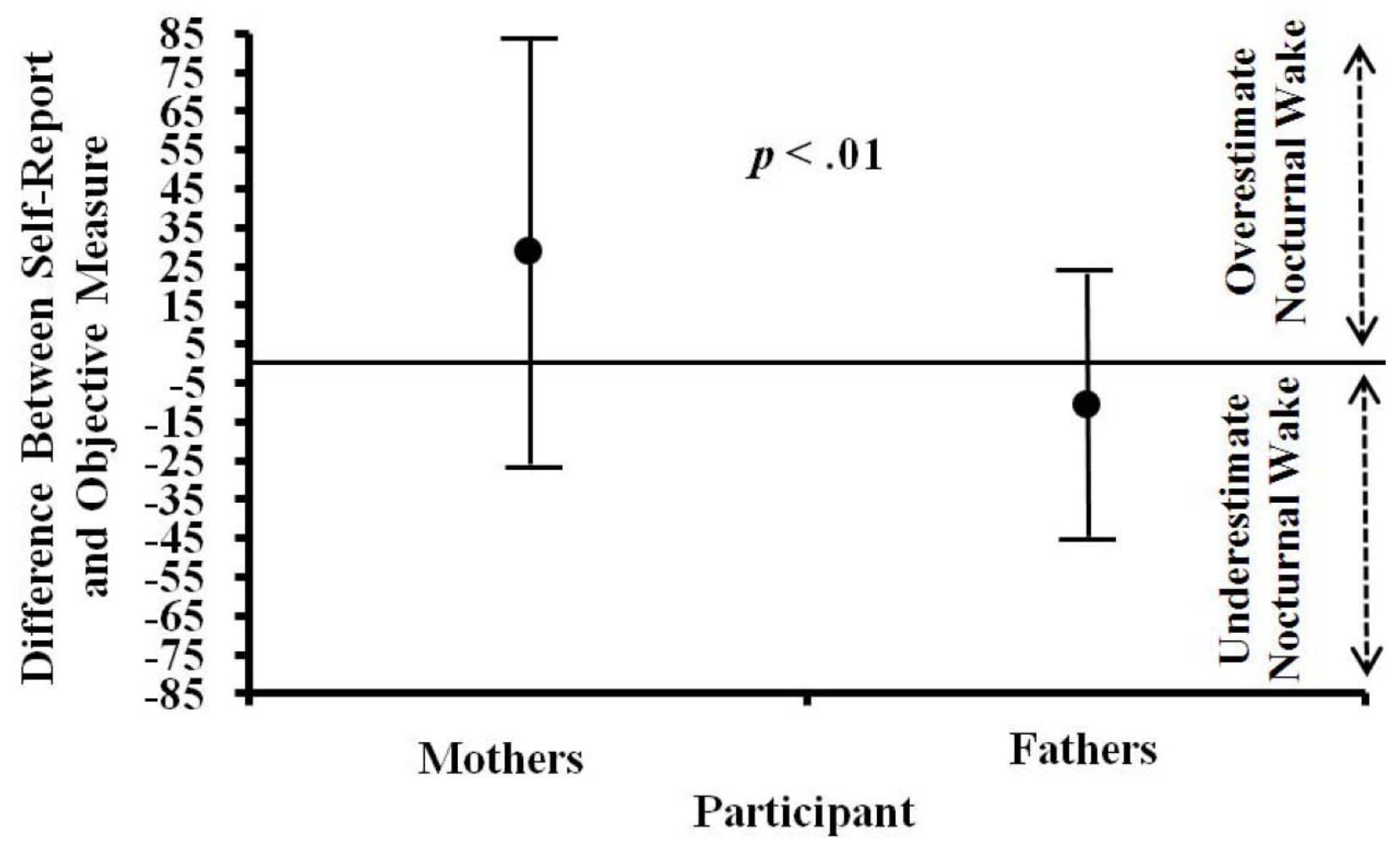


Figure 6 a. Bland-Altman plots for mothers' reports on fathers' nocturnal wake time when compared to fathers' objectively measured nocturnal wake time: arbitrary guide lines at 0,20 , and 40 minute differences.

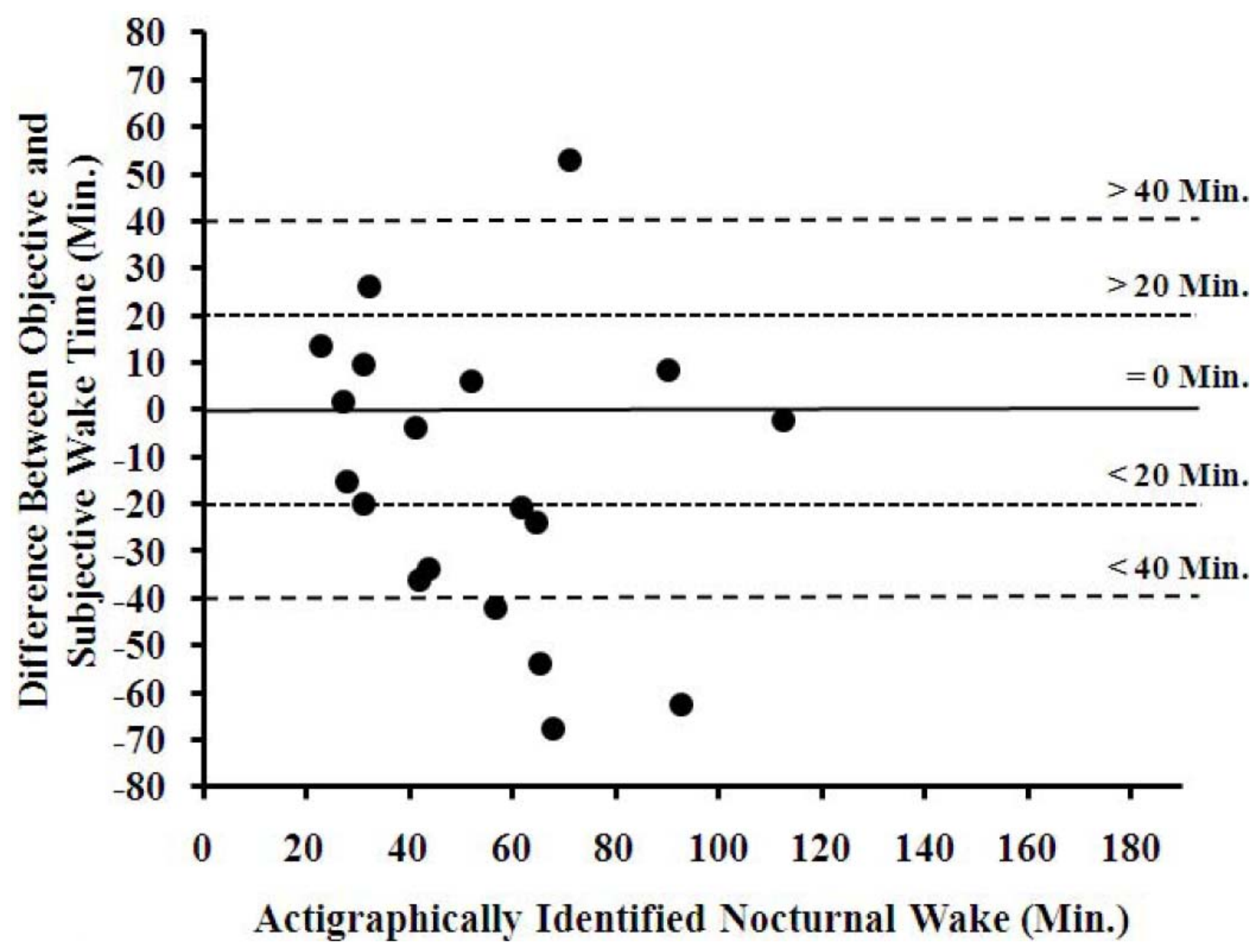


Figure $6 \mathrm{~b}$. Bland-Altman plots for fathers' reports on mothers' nocturnal wake time when compared to mothers' objectively measured nocturnal wake time: arbitrary guide lines at 0,20 , and 40 minute differences.

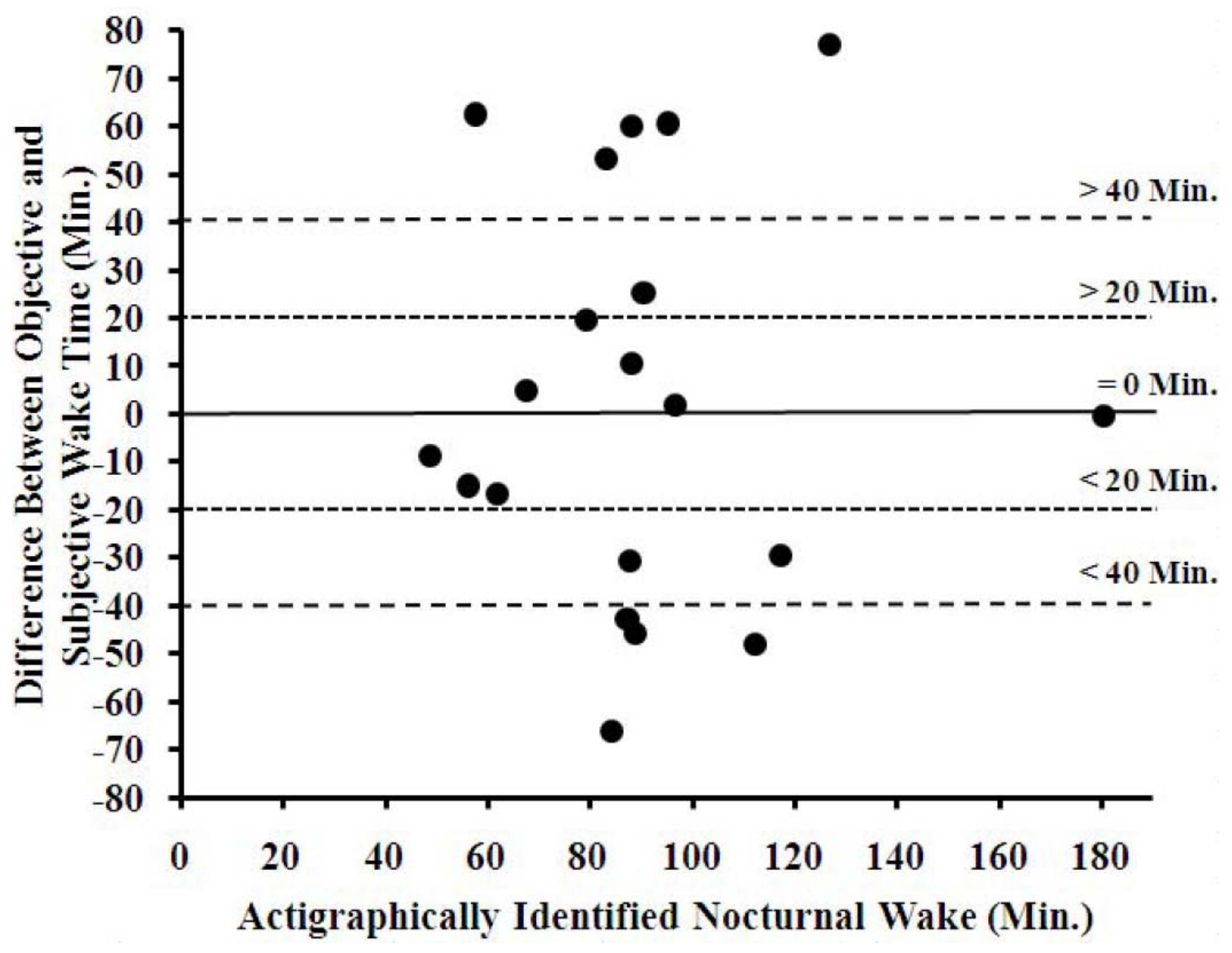


Figure $6 \mathrm{c}$. Plots for mothers' and fathers' reports on partners' nocturnal wake time compared to their partners' objectively measured nocturnal wake time: Means differences and standard deviations

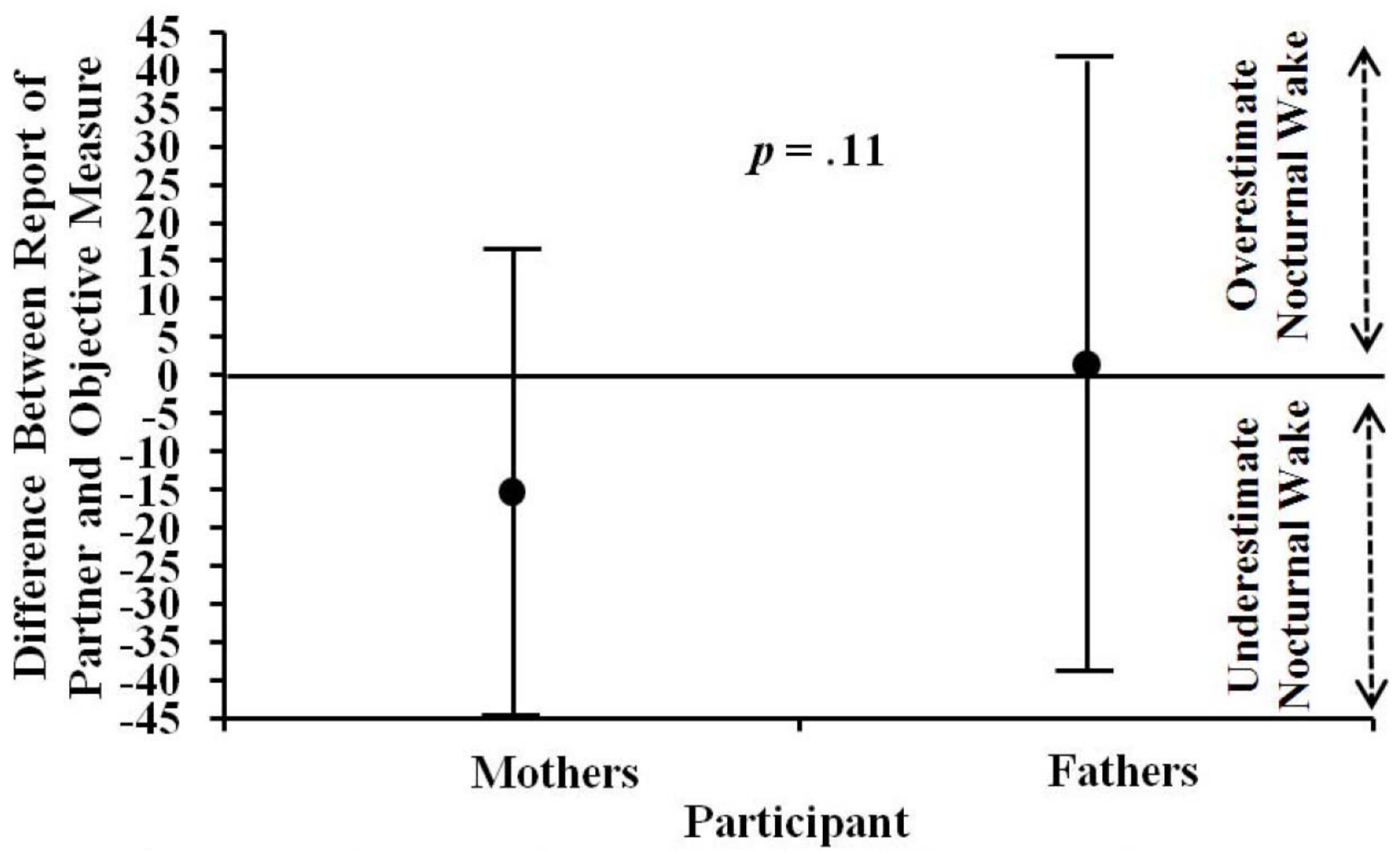


Appendix 1

\section{Community resources for mental health}

Morgantown Mental Health Associates (304) 599-1816

(304) 599-1816

Chestnut Ridge Outpatient Services

(304) 598-4214

Carruth Center for Counseling

(304) 293-4431

Valley Health Care

www.valleyhealthcare.org

(304) 296-1731

Wedgewood Family Practice Inc.

www.wedgewood-fp.com

(304) 599-9400

Valley Community Mental Health Center: Crisis Unit

www.valleyhealthcare.org

(304) 293-3562

Progressive Preventive Health

(304) 225-1177

\section{Community resources for sleep}

Ruby Memorial Hospital Sleep Disorders Center (Morgantown)

(304) 598-4855

The Sleep Center of Mon General hospital (Morgantown)

(304) 599-7934

Fairmont General Hospital Sleep Disorders Laboratory (Fairmont)

(304) 367-7103

Sleep Network of West Virginia (Fairmont)

(304) 367-7103

United Hospital Center Sleep Disorders Center (Clarksburg)

(304) 624-2712 
Appendix 2

If you are routinely robbed of a good night's rest you may have a sleep disorder. This chart lists symptoms associated with several common sleep problems. For each symptom you have, decide how severely or how frequently it affects you, on a 10 point scale. Then check the chart to see whether you should seek treatment. If you experience two or more symptoms, consider moving up to the next recommendation level.

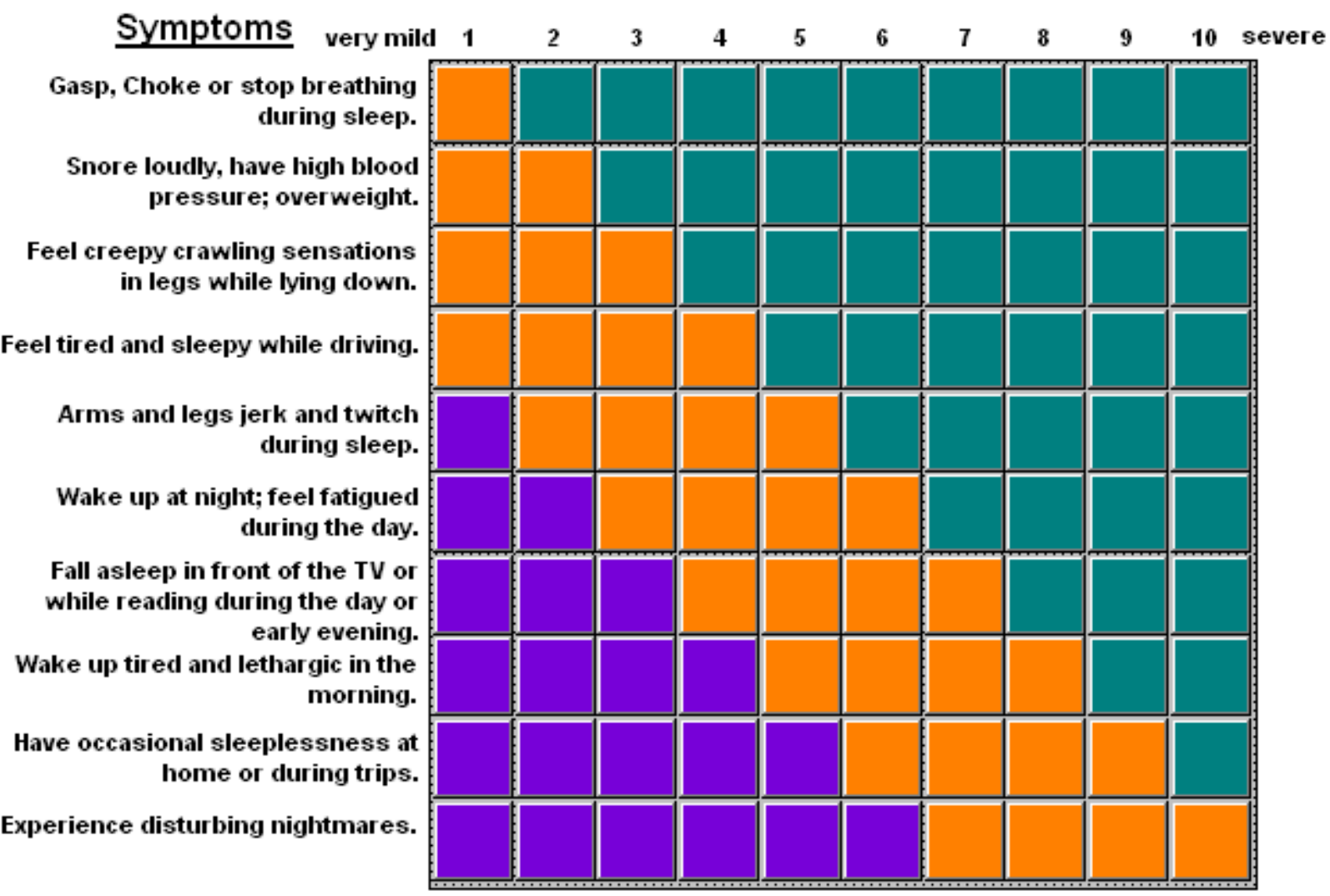


Appendix 3.
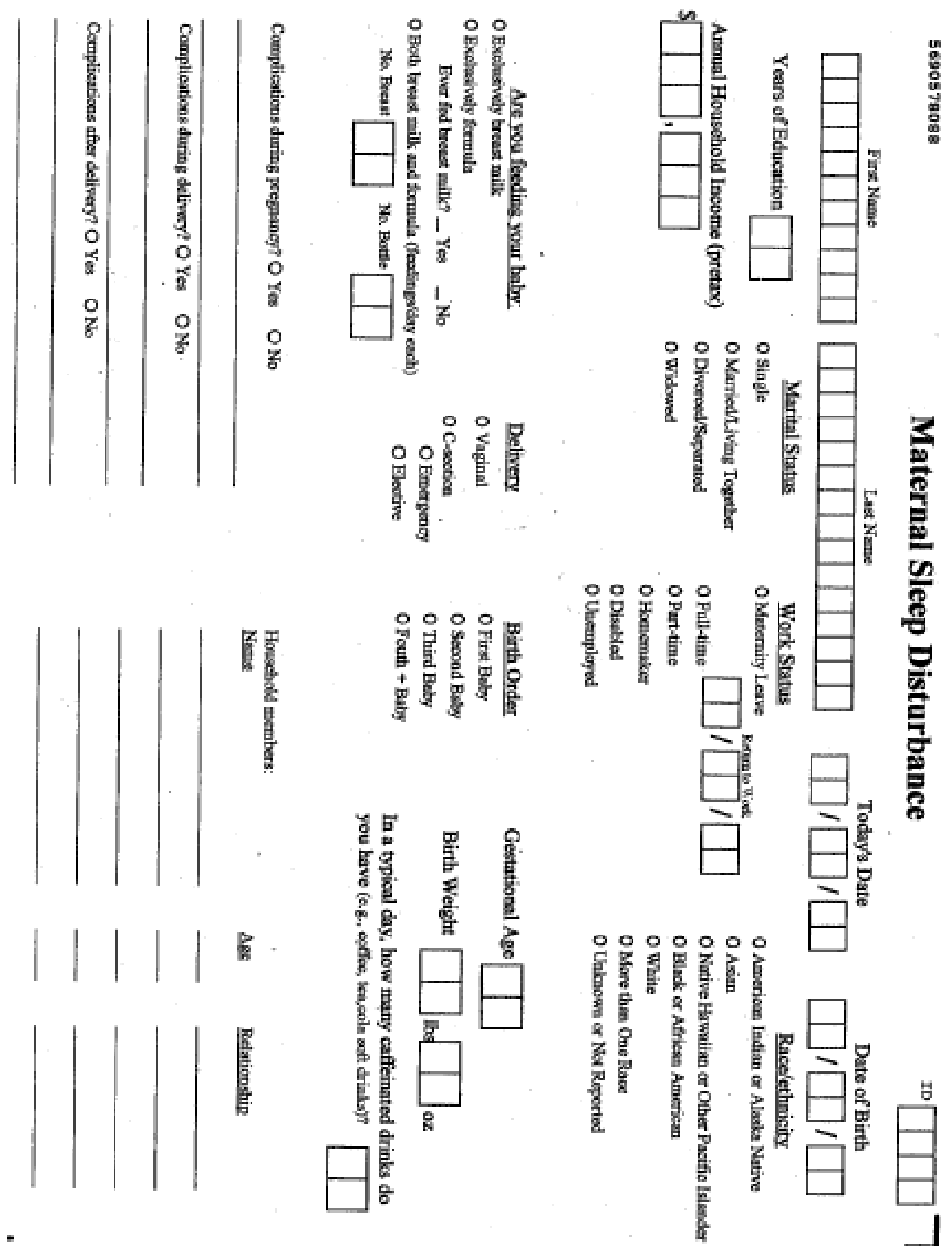
Appendix 4.

\section{Relationship History}

1. Approximately, what was the date and year that you and your partner first met; that is, for your current relationship or otherwise?

Approximate Date:

2. Approximately, what was the date and year that you and your partner began your dating relationship?

Approximate Date:

3. Approximately, what was the date and year that you and your partner began cohabitating (i.e. living together)?

Approximate Date:

4. Are you and your partner married?

-Yes

-No

5. If "Yes", what was the date that you and your partner got married?

Date:

Thank you! 
Appendix 5.

Note: Each of the following ratings will be recorded via the voice note function in the hand-held computer.

\section{Subjective Rating of Nocturnal Wake (Each Morning):}

Describe why you woke up and what you did during each awakening last night.

\section{Subjective Ratings of Sleepiness (Before each MSLT nap opportunity):}

How much time will it take you / your partner to fall asleep during the MSLT? 
Appendix 6

\begin{tabular}{|c|c|}
\hline Mothers & Fathers \\
\hline $\begin{array}{l}\text { 08/29/08: I woke up approx } 4 \text { times during the night, once to feed } \\
\text { and diaper change then } 3 \text { times because I felt that she was } \\
\text { breathing differently and umm was a little paranoid and then } \\
\text { woke up to check to make sure that she was okay, she had some } \\
\text { congestion in her nose so I did some suction. } \\
\text { 08/30/08: Recordings for Friday's wakes. } 1 \text { st wake I fed the baby } \\
\text { and changed diaper, } 2 \text { nd wake I changed the diaper after } \\
\text { [Husband] fed the baby, and 3rd wake was another feed and } \\
\text { diaper change. } \\
\text { 08/31/08: Recordings for Saturday's awakenings. I woke up } \\
\text { approx } 3 \text { times and each awakening was because I had to feed and } \\
\text { then change the baby. } \\
\text { 09/02/08: Recordings for Sunday's wake times. I got up twice } \\
\text { during the night, both times to feed and then change the baby. } \\
\text { 09/02/08: Recordings for Monday's awakenings. I woke up } 2 \\
\text { times during the night. One time was to feed and change the baby, } \\
\text { and the 2nd time I woke up because it was my husband's turn to } \\
\text { feed and change the baby, but I stayed up anyway. } \\
\text { 09/04/08: Recording for Tuesday's awakenings. I got up once } \\
\text { during the night and it was to feed and change the baby. } \\
\text { 09/04/08: Recording for Wednesday's wakening I woke up once }\end{array}$ & $\begin{array}{l}\text { 8/29/08: Last night I woke about } 3 \text { times. All } 3 \text { times were } \\
\text { because the baby was crying I didn't get to sleep until } 4 \text { in the } \\
\text { morning because I had taken a nap earlier in the evening. So, all } 3 \\
\text { times I woke because the baby was crying and my wife did the } \\
\text { feeding and the last time that she fed early in the morning I got up } \\
\text { to console the baby a little bit and walk with her. } \\
\text { 08/31/08: I will be doing two nights worth of recordings I forgot } \\
\text { to do Fri-Sat when I was sleeping and I will also do Sat night into } \\
\text { Sun on this one. Friday night into Sat I woke approx } 3 \text { times each } \\
\text { time because the baby was crying and needed fed, I fed once my } \\
\text { wife fed twice. Both times I woke fully and helped her to burp the } \\
\text { baby whenever she fed. Sat night into Sun. morning I did just } \\
\text { about the same thing. I woke } 3 \text { times, I fed once, she fed twice } \\
\text { and we did about the same thing, I burped the baby one time and } \\
\text { that was it. } \\
\text { 08/01/08: Last night I woke up twice. Once was to feed the baby } \\
\text { and the second time my wife fed the baby, and when I finally got } \\
\text { up I took the baby downstairs so my wife could get some sleep, } \\
\text { and I was up until later on and fell asleep which was on my sleep } \\
\text { log and I also forgot to put my watch back on today after I } \\
\text { showered so it's been off for probably about } 8 \text { hours now. } \\
\text { 09/03/08: I woke twice last night. Once to feed the baby and once } \\
\text { when my wife got up to feed the baby. }\end{array}$ \\
\hline
\end{tabular}




\begin{tabular}{|c|c|}
\hline $\begin{array}{l}\text { 09/05/08: Recordings for Thursday's awakenings. I got up approx } \\
\text { four times last night, } 1 \text { was to feed and the other } 3 \text { were because } \\
\text { she was a little bit active in her sleep and it woke me up. but she } \\
\text { didn't need fed or anything like that she was just kind of um } \\
\text { moving around in her sleep and talking, not talking but actually } \\
\text { just making noises, so I was up about } 4 \text { times. }\end{array}$ & $\begin{array}{l}\text { 01/04/08: I woke up twice last night. Once to feed the baby and } \\
\text { that lasted probably about } 30-45 \text { min and I woke up once again } \\
\text { later that night, but that was when my wife got up to feed the } \\
\text { baby. }\end{array}$ \\
\hline $\begin{array}{l}\text { Couple 2 } \\
\text { 11/18/08: Last night [Partner] never woke up, which is usual. } \\
\text { The really only time he ever woke up is when I go to breastfeed } \\
\text { the baby. I woke up twice last night. I fed the baby both times, } \\
\text { and I was up for about } 6 \text { hours all together }\end{array}$ & $\frac{\text { Couple 2 }}{11 / 18 / 08: \text { [Mother] woke up to feed the baby and I stayed asleep }}$ \\
\hline N/A & $\begin{array}{l}\text { Couple } 3 \\
\text { 09/02/08: The first time I woke up last night I just had to use the } \\
\text { bathroom. } \\
\text { 09/02/08: The first time I woke up last night I just had to use the } \\
\text { bathroom the second time the baby was crying because she was } \\
\text { hungry and then I couldn't go back to sleep for a while, that's all. } \\
\text { 09/03/08: Uh... last night I woke up just to use the bathroom. } \\
\text { 09/04/08: I don't think I woke up at all last night, but I know she } \\
\text { did and she was stuck up for a while, I reckon. } \\
\text { 09/05/08: Last night I slept all the way through the night, I didn't } \\
\text { get up, thank you. } \\
\text { 09/06/08: Last night I woke up because my daughter was crying I } \\
\text { only stayed up for maybe } 5 \text { or } 10 \text { minutes. [Mother] stayed up for } \\
\text { a few hours, she wouldn't go back to sleep last night she slept all } \\
\text { day, but she wouldn't sleep through the night. }\end{array}$ \\
\hline
\end{tabular}




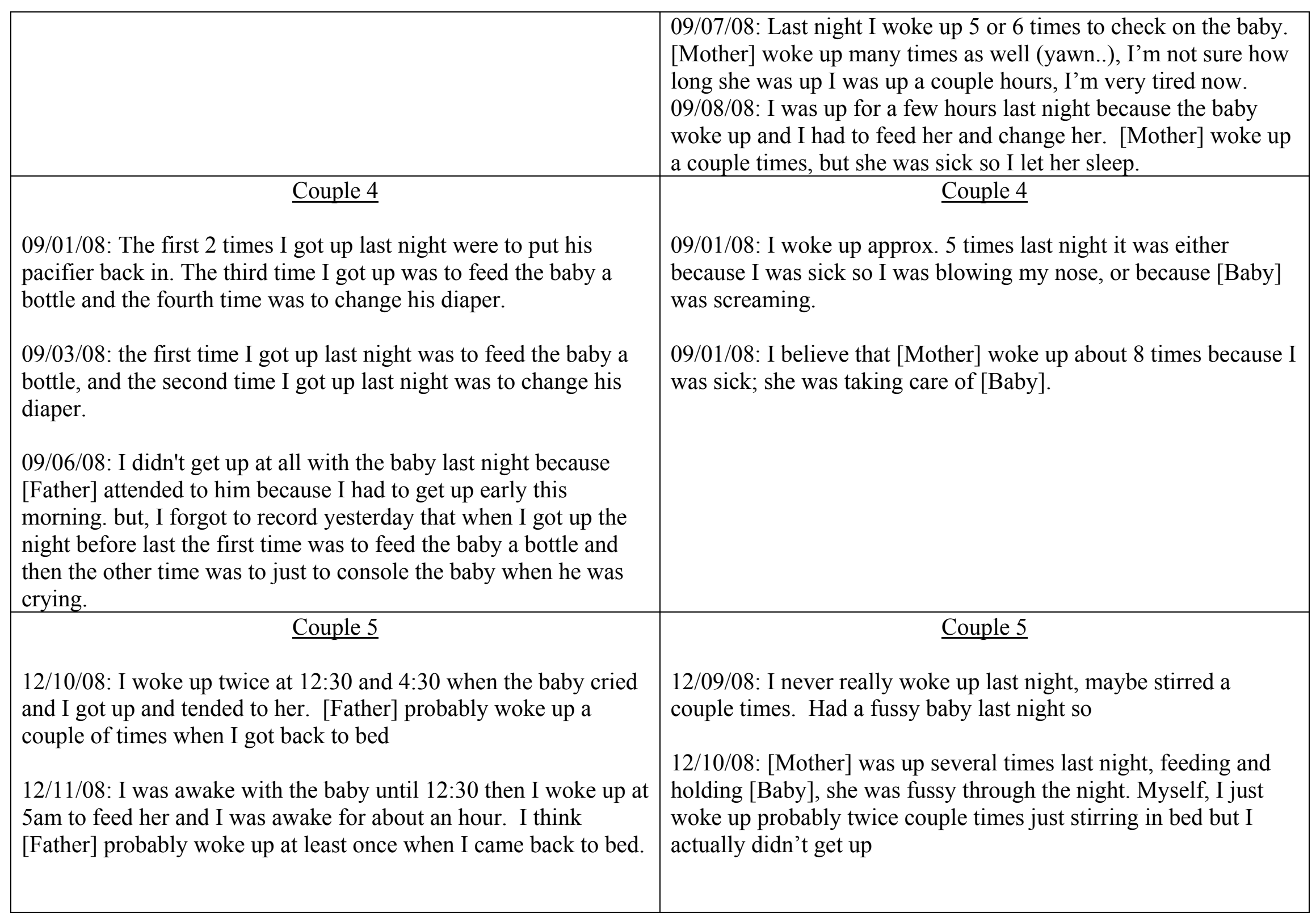


12/12/08: I was awake until 2 and was not woken up again until a little after 7 when the baby woke up and I think [Father] might have woken up a little when I came back to bed around 2

12/13/08: [Father] and I both woke up several times last night and each time was for the baby when the baby would cry, I would get up and take care of her [Father] got up once. I got up several times, I've lost count.

12/14/08: I was awake from 11:30-2:30 then again from 4-5 each time when the baby woke up and [Father] was probably awake for the first half hour of the 11:30-12:00 but probably woke up a few times when I came back in the room.

12/15/08: I accidently did my morning questions before [Father] did this morning. I woke up around 1, 4, and 6 o'clock for a half an hour each time to care for the baby. [Father] woke up around 1 o'clock and was awake with me for a half an hour

\section{Couple 6}

12/11/08: First time I woke up was to move from the couch to the bed and I fed him then every time was just to feed him and I woke up because he was crying.

12/12/08: I woke up twice last night both times because he was crying, and I fed him and then I went to sleep.

12/13/08: Both times I woke up was because he was crying and I fed him and then went back to sleep.

12/14/08: I didn't wake up at all last night, I slept the whole night through but that was probably because the baby was at a babysitters all night.
12/11/08: I would say I woke up a couple times last night, and just as [Mother] was feeding [Baby], woke me up a little bit...never got out of bed. [Mother] I would say probably got up 3-4 times last night, probably up 2-3 hours just feeding and working with [Baby].

12/12/08: Assumed to be a good night for sleep. I think [Baby] slept through the night which meant I believe [Mother] was in the bed all night last night. I anticipate she probably woke up a couple times just to check on her. I believe I woke up a couple times and just stirred in bed, but that was it.

12/13/08: Last night [Mother] spent a lot of time up last night trying to get [Baby] to get some sleep, so kept her up quite bit of night.

\section{$\underline{\text { Couple } 6}$}

12/11/08: I woke up a couple times last night, once was to move....

12/11/08: Moved from the couch to the bed around 3:00 AM then I changed a couple diapers and that would be it.

12/12/08: I did not wake up at all last night, probably because we went to bed around 3:00 AM.

12/13/08: I did not wake up once last night because I was so tired

12/14/08: I did not wake up at all last night; I don't think she woke up either because we had a babysitter. First set of questions 
12/15/08: Every time I woke up last night was because he was crying and I would either feed him and go back to sleep or I'd just sit there and pat his back until he went to sleep

12/16/08: Twice when I woke up I fed him and he went back to sleep and the other times he was just fussy and gassy so I just waited for him to fall asleep or I rubbed his back and went back to bed.

12/17/08: The first two times I woke up were to put him back to sleep cause he woke up wasn't fussy it didn't take long the third time I woke up he was really upset and I had to stay awake with him for a long time

12/18/08: When I woke up it was because he was crying and then I fed him and he went back to sleep

\section{Couple 7}

12/13/08: Fed the baby at 11:00 last night we both got to bed about midnight and woke up at 6:00 in the morning with no interruptions then I fed the baby and it took me 45 minutes, then another 45 minutes to fall asleep and I'm really tired so I'm going back to bed.

12/15/08: I forgot to record this morning about last night. Had a really hard time getting the baby to bed last night so I was up for about two hours trying to get the baby to sleep then I was up for another hour feeding him again later in the morning so every time I was up last night it was for the baby.

$12 / 17 / 08$ : I'm so tired that I forgot to record the questions for my husband. I believe my husband woke up one time for him back was for me and second set for her and the PVT was obviously for me.

12/15/08: I woke up once last night and that was to change a diaper at the beginning of the night.

12/16/08: I woke up once to change a diaper, then another time to put him on my side of the bed

12/18/08: I didn't wake up once last night, I believe [Mother] woke up once or twice just to feed him, then go back to sleep

\section{Couple 7}

12/13/08: I woke up at 8: 00 to go to the bathroom, and then I got up for good at like 20 after eight to go to the bathroom and kind of because of the baby.

12/15/08: I don't think I got up at all last night literally getting up but I think [Mother] woke me up a couple times to tell me the baby was having trouble sleeping. I don't think I got up and hit the thing 10 minutes late (?)

12/16/08: I woke up one time last night [Mother] woke me up a little bit after I went to bed because the baby wouldn't sleep and was fussing so I went over and rocked him and patted his bum until he fell asleep and that only took ten minutes then I put him back to sleep and he slept soundly until this morning 


\begin{tabular}{|c|c|}
\hline $\begin{array}{l}\text { and I believe he was up for about five minutes. I woke up about } \\
\text { five times, first two trying to get the baby asleep then later to feed } \\
\text { the baby, and then I woke up later to get my husband ibuprofen, } \\
\text { and then later to feed the baby again. Again, forgot to record } \\
\text { husband's questions so please insert those for me. } \\
\text { 12/18/08: I got up once last night to feed the baby, was then really } \\
\text { tired because I got to bed late because it took me an hour and a } \\
\text { half to get the baby to fall asleep last night. } \\
\text { 12/19/08: Got up one time last night to change/feed the baby and } \\
\text { put him to sleep, it took a little over an hour. }\end{array}$ & $\begin{array}{l}\text { 12/17/08: I think I woke up one time last night to go to the } \\
\text { bathroom and my back hurt so I took some ibuprofen, I might of } \\
\text { had to take care of the baby last night I can't remember, when I } \\
\text { was sleeping I slept really sound. } \\
\text { 12/18/08: I got up twice last night; both times were to just go to } \\
\text { the bathroom. } \\
\text { 12/19/08: I woke up I think one time last night to go to the } \\
\text { bathroom, it's surprisingly hard to remember how many times } \\
\text { you woke up in the middle of the night. }\end{array}$ \\
\hline $\begin{array}{l}\text { Couple } 8 \\
\text { 12/18/08: I woke up two times last night, both times were to feed } \\
\text { the baby and the night before last I woke up three times, twice to } \\
\text { use the bathroom and once to feed the baby. } \\
\text { 12/19/08: I woke up three times last night, twice to use the } \\
\text { restroom and once to feed the baby. } \\
12 / 20 / 08: \text { I woke up three times last night, once to bathe the baby, } \\
\text { once to feed the baby, and once to use the restroom. } \\
12 / 21 / 08: \text { I woke up twice last night, once to feed the baby and } \\
\text { once to bath the baby. } \\
12 / 22 / 08: \text { I woke up twice last night, once to feed the baby and } \\
\text { once to use the restroom. } \\
12 / 23 / 08: \text { I woke up three times last night, twice to feed the baby } \\
\text { and once to go to the bathroom. }\end{array}$ & Couple 8 \\
\hline
\end{tabular}




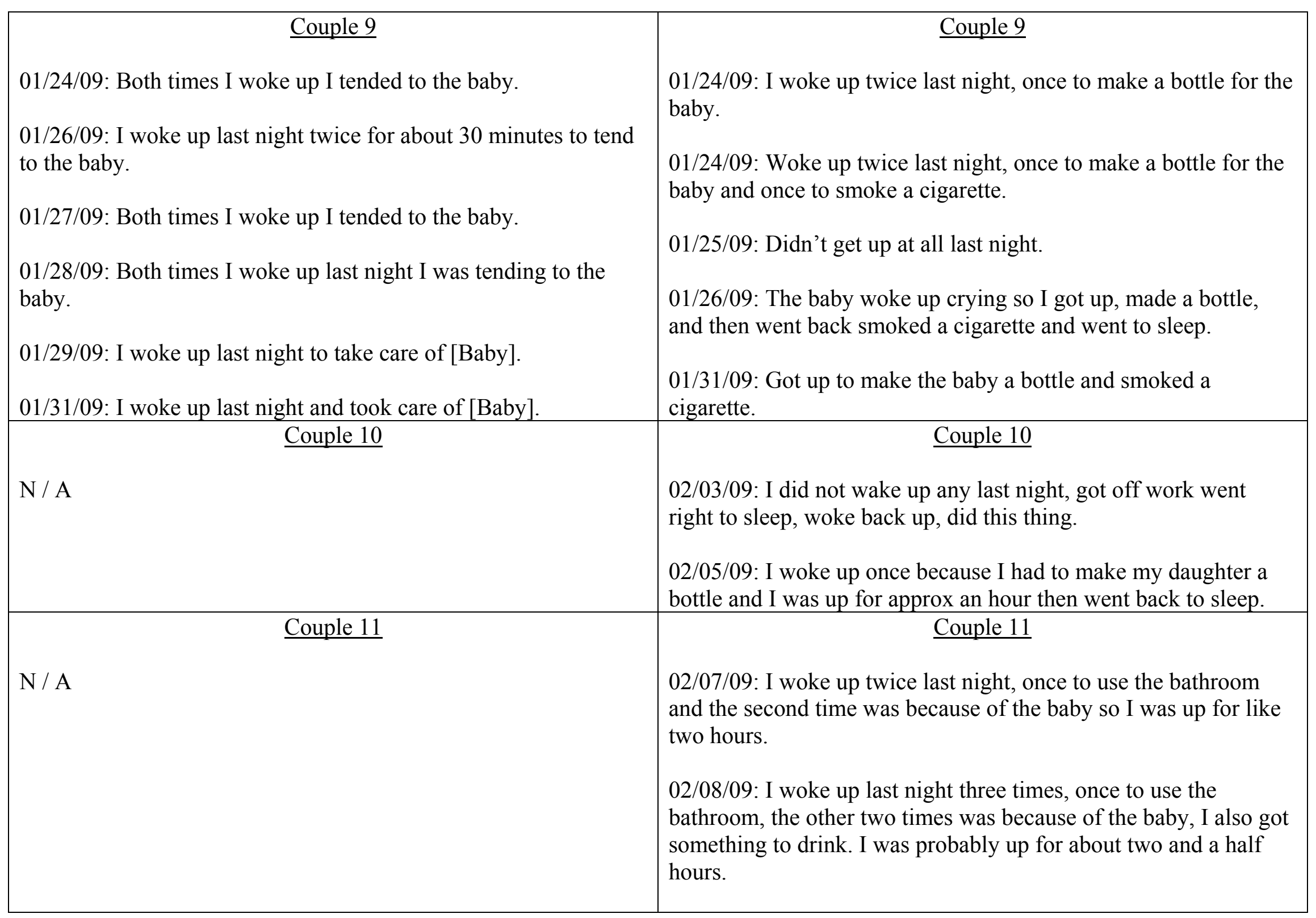




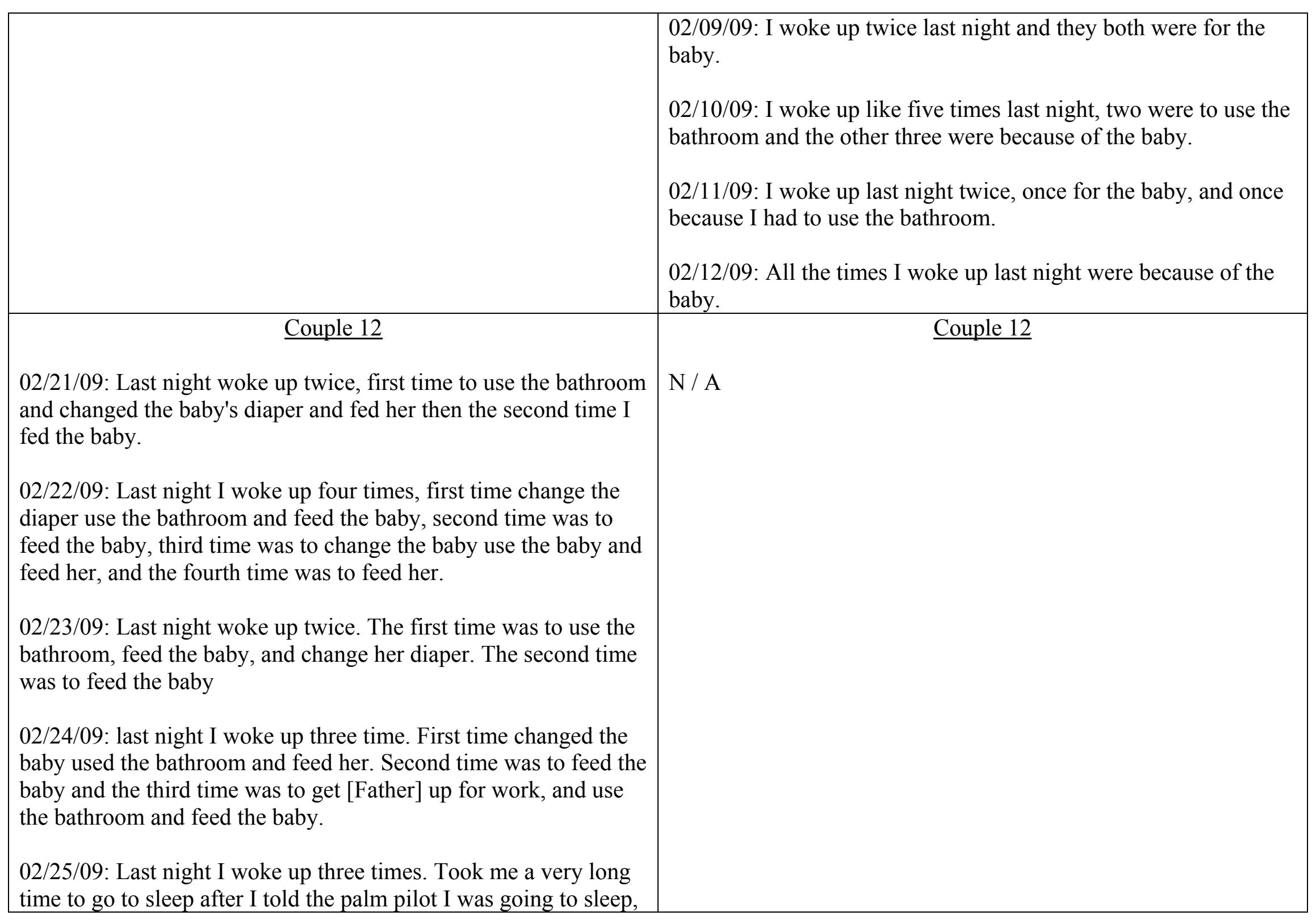




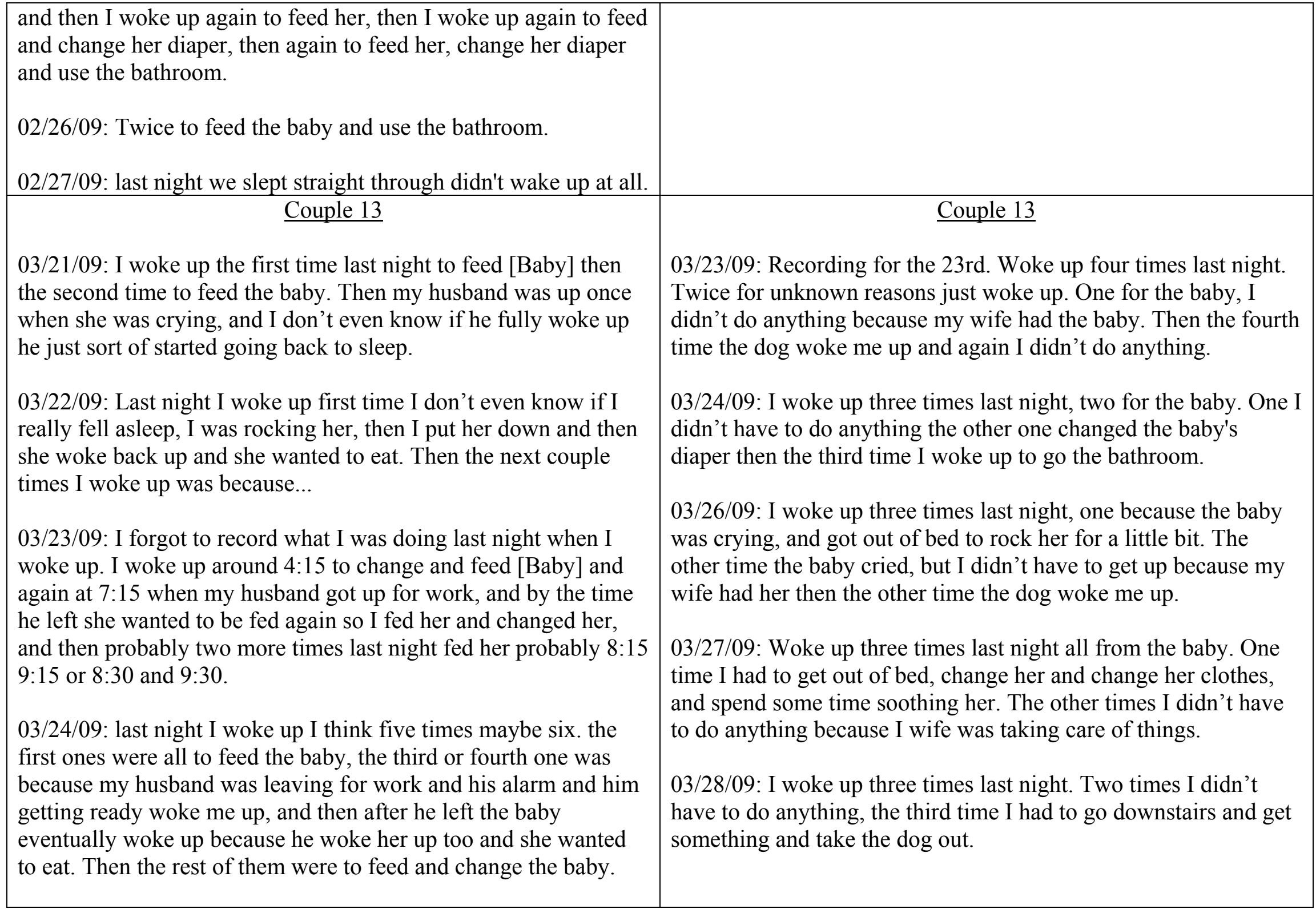




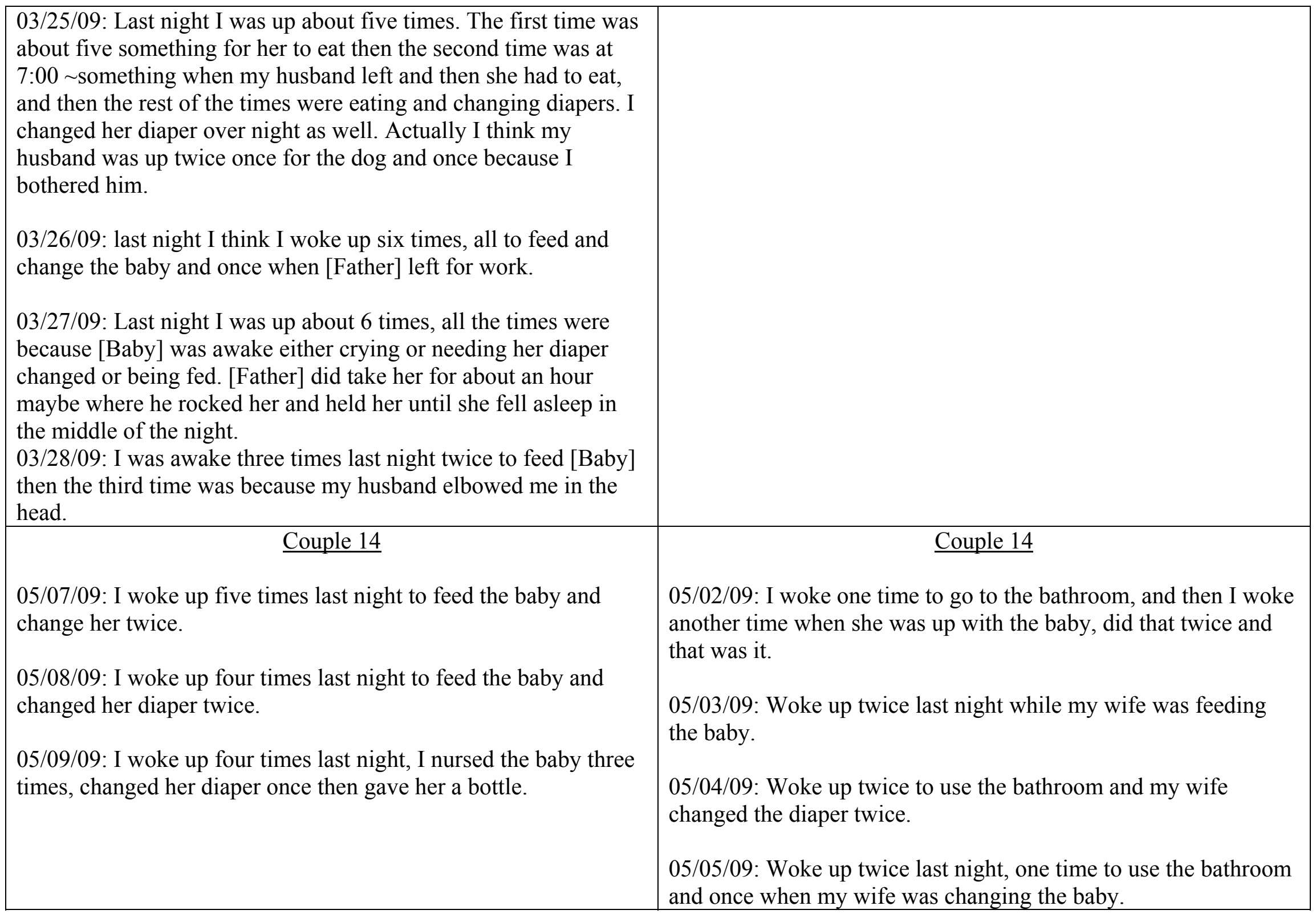




\begin{tabular}{|c|c|}
\hline & $\begin{array}{l}\text { 05/06/09: Got up twice last night, once time to use the bathroom } \\
\text { and the other time to check on my wife and my baby. } \\
\text { 05/07/09: Woke up twice last night, one time to check on my wife } \\
\text { and my baby and another time to use the restroom. } \\
\text { 05/08/09: Woke up three times last night, twice to check on wife } \\
\text { and baby and one time to use the restroom. } \\
\text { 05/09/09: Woke up about } 3 \text { times last night, twice to check on my } \\
\text { wife and my daughter and that's it. }\end{array}$ \\
\hline $\begin{array}{l}\text { Couple } 15 \\
\text { 05/09/09: I woke up last night several times because I needed to } \\
\text { breast feed and I had to pump because we went on a date and I } \\
\text { had extra milk from not feeding him. } \\
\text { 05/12/09: The times that I woke up last night and the last couple } \\
\text { nights (because I've forgotten to voice record) the times I woke } \\
\text { up have either been for breast feeding or pumping. } \\
\text { 05/14/09: I woke up last night to breast feed and to pump and that } \\
\text { was the only time I woke up. } \\
\text { 05/15/09: I woke up last night just to breast feed, didn't get out of } \\
\text { bed for anything else. } \\
\text { 05/16/09: I woke up several times last night because [Baby] } \\
\text { wouldn't sleep, slept too much during the day. Then was hungry } \\
\text { when I woke up so I ate, so I woke up a lot more last night than I } \\
\text { do most nights. }\end{array}$ & $\begin{array}{l}\text { Couple 15 } \\
\text { 05/14/09: Dream like thoughts when I doze off. I fight it but } \\
\text { occur probably whenever I'm sleepy staring at comp screen } \\
\text { midmorning to afternoon I try not to fall asleep. I've been pretty } \\
\text { tired lately so it's not really a good thing for me to be asleep at } \\
\text { work, so I try to stay awake. } \\
\text { 05/15/09: Feeling a little better rested, went to bed at 11:00 only } \\
\text { woke up at 2:00 and 3:00 last night. Running behind, always } \\
\text { seem to sacrifice time for Quality of Sleep. }\end{array}$ \\
\hline
\end{tabular}




\begin{tabular}{|c|c|}
\hline Couple 16 & Couple 16 \\
\hline $\begin{array}{l}05 / 21 / 09 \text { : Last night I woke up about five times, one time to } \\
\text { check on the baby then the rest were because she was hungry or } \\
\text { needed changed. } \\
05 / 22 / 09 \text { : Last night I woke up once to feed the baby and change } \\
\text { her. }\end{array}$ & $\begin{array}{l}\text { 05/21/09: Last night I woke up about three times, I was trying to } \\
\text { comfort my wife. } \\
\text { 05/21/09: Last night I woke up about three times listening to the } \\
\text { baby scream, then I tried to comfort my wife and went back to } \\
\text { sleep. }\end{array}$ \\
\hline $\begin{array}{l}\text { 05/23/09: Last night I woke up once to feed the baby and change } \\
\text { her. }\end{array}$ & $\begin{array}{l}05 / 22 / 09 \text { : Last night I didn't feel that I got a lot of sleep, very } \\
\text { tired when I woke up and very fatigued throughout the whole day. }\end{array}$ \\
\hline $\begin{array}{l}05 / 24 / 09 \text { : Last night I woke up once to feed the baby and change } \\
\text { the baby. }\end{array}$ & $\begin{array}{l}\text { 05/24/09: Last night I woke up twice, once because I heard the } \\
\text { screaming then twice because my back was hurting. }\end{array}$ \\
\hline $\begin{array}{l}05 / 25 / 09 \text { : Last night I woke up once to feed the baby and change } \\
\text { the baby. } \\
05 / 26 / 09 \text { : Last night I woke up three times, once to go to the } \\
\text { bathroom and twice to feed the baby and change the baby because } \\
\text { she was crying }\end{array}$ & $\begin{array}{l}\text { 05/25/09: I woke up a few times last night. The baby was crying } \\
\text { once then I had back pain last night so hard for me to concentrate } \\
\text { and sleep. } \\
\text { 05/27/09: I had decent sleep last night woke up couple times, one } \\
\text { main time to change the diaper. }\end{array}$ \\
\hline $\begin{array}{l}\text { 05/27/09: Last night I woke up about three times, twice to feed } \\
\text { and change her and then one time was just to feed her } \\
\text { 05/28/09: Last night I don't think I woke up at all, the baby slept } \\
\text { through the night so I didn't have to wake up with her. }\end{array}$ & $\begin{array}{l}05 / 28 / 09: \text { I felt that I woke up twice last night. Once specifically } \\
\text { to check on the baby, then twice because I had a lot of pain. }\end{array}$ \\
\hline $\begin{array}{l}\text { Couple } 17 \\
05 / 23 / 09: \text { I woke up because of the baby three times. } \\
05 / 25 / 09: \text { Just woke up to feed the baby twice. }\end{array}$ & Couple 17 \\
\hline
\end{tabular}




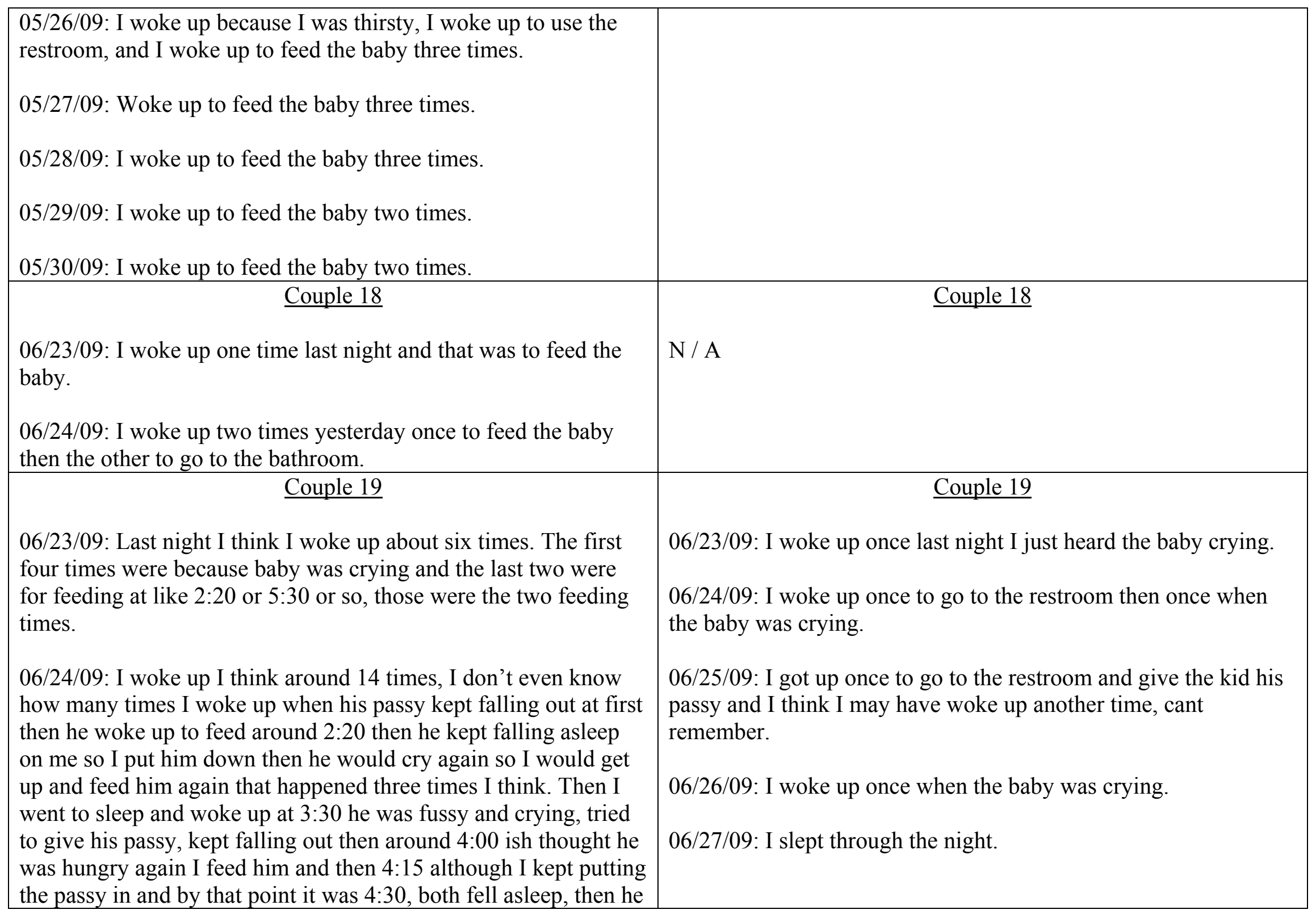


woke up at 5:00 and when I woke up I gave up and handed him over to my husband. No, it was at 6:00 so at 5:00 I think I might have tried to feed him again, then he got back up at 6:00 and I fed him a bottle.

06/25/09: I woke up about six times last night

06/25/09: First time he was crying for food I fed him. Second time he was hungry too, I fed him. Third time I think I thought he was hungry but when I got up just gave him passy and went back to bed lasted for ten minutes, maybe. I got back up, fed him, went back to sleep, then the next time I woke up and I told [Husband] to go in and hang out with the baby and feed him. Then the next time I woke up to feed him

06/26/09: I woke up about seven times last night. The first time a friend texted me about 12:45, then 1:45 to feed the baby and then I think another time when I heard him crying. I put the passy in then at 5:50 I woke to feed him and then I had [Father] get up at 6:00 and stay up with him. I woke two other times when I heard him crying when I was sleeping, and then I woke up about 8:40.

06/27/09: Last night I woke up each time to feed the baby, 12:30 then [Father] woke me up, 3:30, and 6:45, and I fed him. Then there were two other times I got up because I thought he was choking so I got out of bed.

06/28/09: I got up three times last night each time was to feed the baby.

06/29/09: I actually have no clue how many times I woke up last night. I think I woke up three times to feed him, but I think somewhere between 10-14 times; I wrote down 14 times. The
06/29/09: I woke up five or six times last night the baby was fussy.

06/30/09: I woke up a couple times to feed the baby and give him his passy. 


\begin{tabular}{|c|c|}
\hline & \\
\hline Couple 20 & Couple 20 \\
\hline $\begin{array}{l}\text { 07/03/09: I got up twice during the night both times were to care } \\
\text { for the baby. } \\
\text { 07/04/09: I woke up three times last night the first time I didn't } \\
\text { get up I just woke up when [Father] got out of bed the other } 2 \\
\text { times I got up both times to take care of the baby. } \\
\text { 07/05/09: I was awake twice last night the first time my cat woke } \\
\text { me up briefly and the second time I actually got up and cared for } \\
\text { the baby. } \\
\text { 07/06/09: I only woke up one time last night and I got up to care } \\
\text { for the baby. } \\
\text { 07/07/09: I woke up twice last night the first time to care for the } \\
\text { baby and the second time briefly when my cat jumped up on me. } \\
\text { 07/08/09: I woke up twice last night and both times I got up to } \\
\text { take care of the baby. } \\
\text { 07/09/09: I woke up twice last night the first time was to care for } \\
\text { the baby and the second time I woke up because I heard my } \\
\text { husband get up. }\end{array}$ & $\begin{array}{l}\text { 07/03/09: I woke up at 2:15 I don't know why. } \\
\text { 07/03/09: I woke up at 2:15 3:15 3:45 5:20 and 7:30. [Mother] } \\
\text { was up at } 3: 15 \text { and } 7: 30 \text { the other times I don't know why. } \\
\text { 07/04/09: I woke up four times last night } 3: 15,4: 15 \text {, one time } \\
\text { when [Mother] was feeding the baby, and one other time but I } \\
\text { don't recall what the time was. } \\
\text { 07/05/09: I woke up like four times last night, one time at } 3: 00 \text { for } \\
\text { 15 minutes I don't remember the other times. } \\
\text { 07/06/09: I woke up around } 2: 15 \text { for no reason. I woke up at } 3: 30 \\
\text { because [Mother] was feeding baby, at } 4: 45 \text { because dog woke } \\
\text { me up, and 7:45 because time to wake up. } \\
\text { 07/08/09: I woke up at 2:30, 4:30, and } 6: 30 \text { not sure why, I just } \\
\text { felt like waking up I guess but I went right back to sleep. } \\
\text { 07/09/09: I woke up four times last night at } 2: 00,3: 00,5: 00 \text {, and } \\
\text { 6:00; I think not for very long at 5:00, I had to go to the } \\
\text { bathroom. }\end{array}$ \\
\hline $\begin{array}{l}\text { Couple 21 } \\
\text { 07/18/09: I woke up once when [Father] came to bed. } \\
\text { 07/18/09: I woke up three times once when [Father] came to bed } \\
\text { another time to feed baby, and another time just to check the tim }\end{array}$ & $\begin{array}{l}\text { Couple 21 } \\
\text { 07/19/09: I woke up twice during the night both times were to } \\
\text { soothe and put our daughter back to bed for about a total of a half } \\
\text { an hour. }\end{array}$ \\
\hline
\end{tabular}


and go to bathroom.

07/19/09: I woke up two times both times were to feed and care for the baby.

07/20/09: I woke up three times last night and all three of them were to care for the baby or feed her.

07/21/09: I woke up two times last night both times were to feed and care for the baby.

07/23/09: I woke up one time last night to feed the baby.

07/24/09: I woke up three times last night two times were to feed the baby and one time was to pump food for the baby for the next day.

07/25/09: I woke up two times last night one time to feed [Baby] and the other time was to get her to go back to sleep.
07/20/09: I woke up twice during the night to take care of [Baby] for a total of about one hour.

07/22/09: I did not wake up during the night as [Baby] slept for five consecutive hours.

07/23/09: I did not wake up during the night. 
Sleep and Sleepiness

125

Regina Butka's (grandmother) traditional Polish deep fried pastry recipe from her original cookbook: Paczki (pronounced: punch-key)

Paczhí

Polish claughnuts

lnof

$1 / 2$ C - I c sulut creme (Cluke warm

1 yent -2 yeast dry

5 yeete -10 egq yoiks
$1 / 2$ tep $-k T$ salt

$21 / 2 T-5 T$ hooter

21/4c-41/2 c flow

12 Thum - I T reen ood /T araila

Sougar - 6 T sugar

Leat. creme to lecke warm-dupest in

mire egg jalk t sult beat until thick yellow calat

Creme litter a ougar

Ade jeist clessolved in tlspsugar

en cremer micuad lagether

Anead until blisters let rise in warmolace

Cut raunds rise fing invil

John $\mathrm{H}$.

ned by John $\mathrm{H}$.

Hagen

Department, email=John.
Hagen@mail.wvu.edu, $c=U s$
Date: 2010.04.23 12:53:18 